A study of interaction forces at the solid-liquid interface using Atomic Force Microscopy

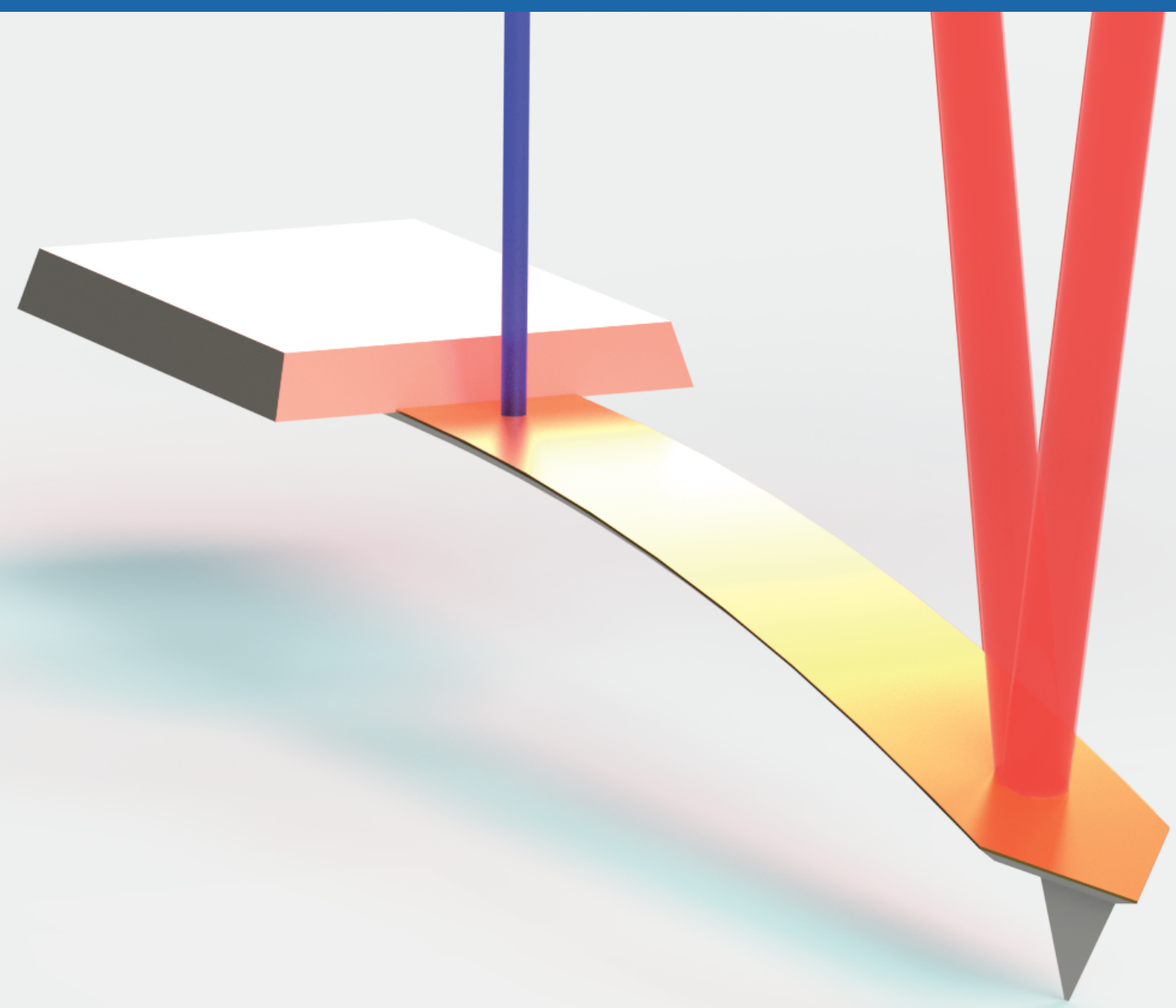

Fei Liu 


\section{A study of interaction forces at the solid-liquid interface using Atomic Force Microscopy}

Fei Liu 


\section{Graduation committee:}

\section{Chairman:}

Prof. dr. ir. J.W.M. Hilgenkamp University of Twente

\section{Promotor:}

Prof. dr. F. Mugele University of Twente

\section{Assistant promotor:}

Dr. ir. H.T.M. van den Ende University of Twente

\section{Members:}

Prof. dr. ir. H.J.W. Zandvliet University of Twente

Prof. dr. J.T.C. Eijkel

University of Twente

Prof. dr. S.J.G. Lemay

University of Twente

Prof. dr. E. Vlieg

Dr. K. Voitchovsky

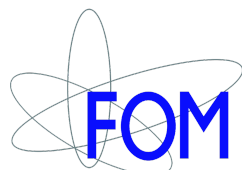

The research for this dissertation was carried out at the Physics of Complex Fluids group, University of Twente, Enschede. This work has been supported by the Foundation for Fundamental research on Matter (FOM), which is financially supported by the Netherlands Organization for Scientific Research (NWO).

Title: A study of interaction forces at the solid-liquid interface using Atomic Force Microscopy

Author: Fei Liu

ISSN: $\quad 978-90-365-4131-2$

DOI: $\quad 10.3990 / 1.9789036541312$

Copyright (c) Fei Liu 2016. All rights reserved.

Cover designed by Aram Klaassen.

Printed by GVO drukkers \& vormgevers B.V. 


\title{
A STUDY OF INTERACTION FORCES AT THE SOLID-LIQUID INTERFACE USING ATOMIC FORCE MICROSCOPY
}

\author{
DISSERTATION
}

to obtain

the degree of doctor at the University of Twente, on the authority of the rector magnificus,

Prof. dr. H. Brinksma,

on account of the decision of the graduation committee,

to be publicly defended

on Thursday, the $19^{\text {th }}$ of May 2016 at 16:45

by

Fei Liu

born on the $10^{\text {th }}$ of April, 1986

in Jiangsu, China 
This dissertation has been approved by:

Prof. dr. F. Mugele (Promotor)

Dr. ir. H.T.M. van den Ende (Assistant promotor) 


\section{Contents}

1 Introduction 1

1.1 Dissertation Outline . . . . . . . . . . . . . . . . 3

2 Forces at the solid-liquid interface: techniques and interpretations $\quad \mathbf{7}$

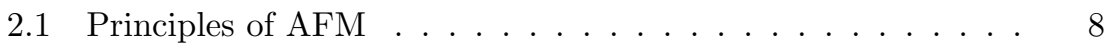

2.2 Challenges with AFM force spectroscopy in liquid . . . . . . . . . . . . . . . 16

2.3 Thin liquid film mediated forces

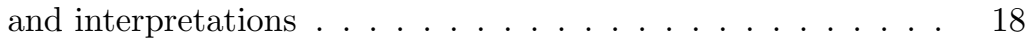

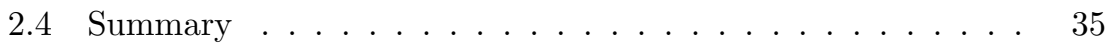

3 Atomic force microscopy of confined liquids using the thermal bending fluctuations of the cantilever 43

3.1 Introduction . . . . . . . . . . . . . . . . . . 4 44

3.2 Methods . . . . . . . . . . . . . . . . . . . . 46

3.3 Data analysis . . . . . . . . . . . . . . . . . . . 5 50

3.4 Results . . . . . . . . . . . . . . . . . . 50

3.5 Discussion . . . . . . . . . . . . . . . . 56

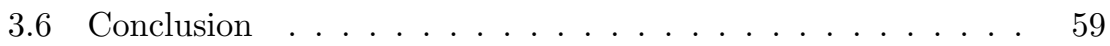

3. A Appendix ....................... 61

4 Amplitude modulation Atomic Force Microscopy, is acoustic driving in liquid quantitatively reliable? $\quad \mathbf{7 3}$

4.1 Introduction . . . . . . . . . . . . . . . . . . 74

4.2 Principles of force spectroscopy $\ldots \ldots \ldots \ldots \ldots$. . . . . . 77

4.3 Procedures and analysis $\ldots \ldots \ldots \ldots$. . . . . . . . . . 81

4.4 Results...................... . . 86 
4.5 Discussion . . . . . . . . . . . . . . . . . 94

4.6 Conclusion . . . . . . . . . . . . . . . . . . . . . . . . . . . 97

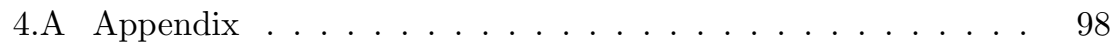

5 Viscous dissipation in overlapping electric double layers 111 5.1 Introduction . . . . . . . . . . . . . . . . . 112

5.2 Methods ........................ 117

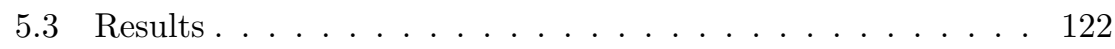

5.4 Discussion . . . . . . . . . . . . . . . . . . . . . . . . . . . . . . . . 137

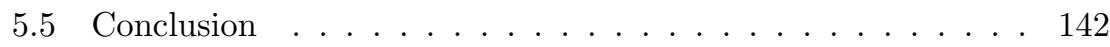

5.A Appendix ...................... 143

$\begin{array}{ll}\text { Summary } & 151\end{array}$

$\begin{array}{lc}\text { Samenvatting } & 153\end{array}$

$\begin{array}{ll}\text { Publications } & 157\end{array}$

$\begin{array}{ll}\text { About the Author } & 159\end{array}$

$\begin{array}{lr}\text { Acknowledgments } & 161\end{array}$ 


\section{Chapter 1}

\section{Introduction}

Interesting phenomena take place when solids meet liquids. For instance, water spreads in a thin film over most metal oxides. But it hardly happens to non-polar substrates, such as dew on blades of grass. Here the water molecules stick together, strongly enough to form droplets. These wetting and non-wetting phenomena are easily observed at the macroscopic scale. However, the underlying interactions between a liquid and a solid surface are often elusive.

Understanding and controlling interfacial phenomena are of crucial importance for many processes in nature and industry, such as adhesion, adsorption, corrosion, filtration and lubrication. It has always been a topic of intense research in many disciplines, including colloid science, electrochemistry, nanofluidics, oil recovery, and mechanical engineering. For instance, the energy cost originating from both solvation and surface charge dictates the stability of colloidal suspension against aggregation [1]. The electrochemistry at interfaces is of interest for the development of novel energy conversion methods and storage devices [2]. Controlling a flow liquid through a nanochannel is crucial for the emerging nanofluidic technology and its applications, such as lab-on-chip devices. In oil recovery, it is critical to be able to migrate adsorbed organic matter from the rock surface [3]. In machinery, the performance of a lubricant is the limiting factor that determines the life of gears and bearings.

Near an interface liquids need to adopt different structures than in the bulk due to the presence of the interface and/or the specific interactions between the liquid and the other phase, being it a solid substrate or another liquid or gas. In the case of wetting, the liquid molecules adsorb onto the 
surface. They are not randomly distributed, but have a layered structure. The density profile (normal to the surface) of the liquid is oscillatory with a periodicity comparable to the molecule diameter, analog to the radial distribution of solvent molecules in a solvation shell of a solute [4]. It is usually several molecular layers thick. Beyond this range, the effect of the surface dies out and hence the liquid's (unorganized) structure remains intact. For the non-wetting case, the liquid is repelled from the surface. It leads to depletion of liquid at the interface, where surface nanobubbles (nanoscopic gaseous domains) may emerge [5].

The solid surface influences the interfacial liquid and in turn the surface groups are open to reaction with the liquid. The surface may dissolve or grow. The surface groups may be partially dissociated, such as hydroxyl groups in water. The surface may acquire charges through adsorption or desorption of ionic species. In fact, most surfaces immersed in water become charged, such as mineral surfaces, metal oxides and biological membranes. The charged surfaces attract counter-ions from the liquid phase. As a result, an electric double layer (EDL) is formed [6]. The thickness of the EDL can range from one to a few hundred nanometers, depending on the ion concentration. The electric field in the EDL influences the orientation of a molecular dipole in liquid, such as water.

As mentioned above, liquid near a substrate may structurally differ from the bulk liquid and may have complex interactions with the substrate. This must have consequences for the dynamic properties of the interfacial liquid. For example, diffusion of liquid molecules may be hindered by surface affinity. The viscosity of interfacial liquid may be changed due to electric-field-induced orientation of liquid molecules. Under confinement, liquid may transform into crystalline states and hence its viscosity increases by several orders.

The work in this thesis is devoted to elucidating the properties of solidliquid interfaces by measuring thin liquid film mediated forces between two solid substrates at nanoscale separations. In particular, we are interested not only in the conservative forces but also in the hydrodynamic dissipation at solid-liquid interfaces and how these interactions are related to the interfacial structure.

There are various types of forces between two separated surfaces in liquid. In the continuum regime, the conservative forces are well described by the DLVO theory (named after Derjaguin, Landau, Verwey, and Overbeek). The DLVO theory accounts for the van der Waals force and the electrostatic force between two substrates in close proximity. In the non-continuum regime, the solvation forces (hydration force for water) are dominant and they are not yet fully understood. A general description of the solvation forces is still 
missing, and it stays an unresolved issue in physical chemistry.

Hydrodynamic drag force is imposed when two surfaces approach (or move across) each other under liquid. In the continuum regime, analytical descriptions for the hydrodynamic dissipation are available. However, it is still controversial on how a molecularly thin liquid film under confinement responds to shear or compression. For flow across EDLs, the hydrodynamic dissipation is coupled with electrostatics, leading to electro-hydrodynamic lubrication. It is still unclear to what extent electrokinetics influences the flow.

It is technically demanding to probe forces at solid-liquid interfaces in the nanoscale. It requires an accurate control of surface separation and a high-resolution in interaction forces. In this study, we use atomic force spectroscopy (AFM) [7] to address these interactions. An AFM uses a microscale cantilever with a tip at the end to sense the forces between the tip and the substrate. We directly measure the conservative and dissipative interactions at solid-liquid interfaces as a function of liquid film thickness by bringing a cantilever to the substrate at a controllable rate.

AFM has unique advantages. It is able to offer surface topography with Ångstrom scale resolution and to measure interactions with piconewton resolution. Furthermore, AFM force spectroscopy has been successfully applied to measure DLVO forces. Nevertheless, the techniques for the measurements on hydrodynamic dissipation are still under development. For instance, contradictory observations have been reported on dissipation in confined liquids.

\subsection{Dissertation Outline}

As introductory lines, in this thesis we will investigate the conservative and dissipative interactions between an AFM tip and a substrate as a function of the properties of the surrounding solution. Moreover, we explore several spectroscopic techniques to measure these interactions accurately and efficiently.

In Chapter 2, we introduce the principles of AFM force spectroscopy and forces at solid-liquid interfaces. If available, theories for each type of force are introduced with a review of experimental results. We show that it is still a challenge to conduct reliable AFM force spectroscopy in liquid. The dynamic properties of nano-confined liquids are still unresolved. We also describe the current status of EDL modelling and sketch how to use AFM to investigate electro-hydrodynamics in EDL.

In Chapter 3, we explore a 'non-conventional' AFM force spectroscopy: 
the thermal noise spectroscopy (TNS). It makes use of the thermal fluctuations of a cantilever. With this technique, one is able to avoid issues with ambiguity in the driving force of the cantilever. We theoretically evaluate the accuracy of the simple harmonic oscillator (SHO) approximation for TNS. It turns out it is sufficient and reliable. TNS is further validated by measuring the viscosity of a non-polar liquid in the bulk, using a colloidal probe, with accuracy of approximately $20 \%$. With a nanoscale tip, we observe oscillatory dissipation in a thin film of a few molecular layers thick. Despite its reliability, it has an obvious disadvantage-it is very slow. Measuring one approach curve takes a few seconds. Therefore, after establishing TNS, we examine the reliability of piezo excited amplitude modulation AFM (AM-AFM) force spectroscopy in liquid, which is most widely used and faster.

In Chapter 4, we compare the experimental results from both TNS and piezo-excited AM. Using both techniques, we measure DLVO forces, solvation forces, and the hydrodynamic dissipation in aqueous electrolytes. We find that piezo-excited AM is quantitatively reliable if the fluid mediated excitation is taken into account for modeling the dynamics of the cantilever. Otherwise, artifacts are observed in the resulting viscous dissipation. Oscillatory dissipation in hydration layers is consistently observed with both TNS and AM-AFM.

In Chapter 5, we show the experimental results on viscous dissipation in overlapping electrical double layers. The viscous dissipation is correlated with the charge density in the diffuse layer. We try to explain viscous dissipation due to electrostatic effects by considering both the electro-viscous and visco-electric effect. The electro-viscous effect accounts for the enhanced dissipation due to the presence of the excess ion concentration in the electrolyte film, while the visco-electric effect deals with the viscosity enhancement of solvent (water) due to the strong local electric field in the film. With these effects we can qualitatively explain the observed behavior. 


\section{Bibliography}

[1] Lyklema J 1994 Colloids Surfaces A: Physicochem. Eng. Aspects 9241

[2] Arico A S, Bruce P, Scrosati B, Tarascon J M, and Van Schalkwijk W 2005 Nat. Mater. 4366

[3] Kumar N, Wang L, Siretanu I, Duits M, and Mugele F 2013 Langmuir 295150

[4] Israelachvili J 1991 Intermolecular and Surface Forces 2nd ed (Academic press, London)

[5] Borkent B M, de Beer S, Mugele F, and Lohse D 2010 Langmuir 26260

[6] Lyklema J 1995 Fundamentals of Interface and Colloid Science ( Academic press, London)

[7] Binnig G, Quate C F, and Gerber Ch 1986 Phys. Rev. Lett. 56930 



\section{Chapter 2}

\section{Forces at the solid-liquid interface: techniques and interpretations}

Solid-liquid interfaces are of interest not only from a fundamental point of view, because interfaces show rich spectrum of physical and chemical properties, but also from a more practical point of view because of its economical significance in numerous industrial applications.

It is interesting to know how the interface is structured and how its structure is linked to specific functions. The interfacial structures are often nanoscopic and heterogeneous, which makes it challenging to study solidliquid interface phenomena. Various techniques have been applied for studying solid-liquid interfaces, such as vibration spectroscopy, electrokinetic techniques, and force spectroscopy. These techniques offer complementary information on different length and time scales, such as vibration of interfacial water molecules by sum-frequency generation, ion adsorption by X-ray scattering, ellipsometry, atomic force microscopy (AFM), and surface force apparatus (SFA), surface charge by titration, zeta potential, AFM and SFA, and surface topography by AFM and scanning tunneling microscopy (STM).

The research presented in this thesis aims at improving the understanding of solid-liquid interfaces, using AFM force spectroscopy. AFM is the only tool that offers both atomic-resolution topography and nanoscopic force spectroscopy. By the use of AFM force spectroscopy, we study the forces mediated by a thin liquid film between two objects, in our case an AFM 
tip and a substrate. Tremendous achievements have been made with AFM force spectroscopy at solid-liquid interfaces. However, there are still many unresolved mysteries. Among others, the following questions are still open:

- Is there a general description of the solvation force for all systems?

- How does a molecularly thin liquid film under confinement respond to shear or compression?

- How do the interfacial charges affect flow of electrolyte over a surface?

Below, we first introduce the the principles of AFM force spectroscopy. Then we present how the measured interaction forces are interpreted.

\section{$2.1 \quad$ Principles of AFM}

An AFM is a device that uses a cantilever with a conical tip to sense forces between its tip and the sample. Its invention had a profound impact on the development of nano science and technology. The first AFM was invented by Binnig, Quate and Geber [1] in 1986, shortly after the arrival of its siblingSTM. The AFM inventors envisioned

'a general-purpose device that will measure any type of force; not only interatomic forces, but electromagnetic forces as well.'

This vision has become a reality. The AFM and its offspring (see Fig. 2.1) have been widely used in many disciplines. It is flexible and versatile. It can be operated in very high vacuum, air and liquid. Moreover, the technique does not show any restrictions on conductivity and transparency of samples. Such flexibility is often unavailable with its counterparts. For instance, STM only works with conductive materials.

As a microscope, AFM is able to unveil atomic structures on surfaces in vacuum [3] and recently even in liquid $[4,5]$. As a spectroscope, it has the capability to measure the forces between two micro objects at the nanoscale, which is referred to as force spectroscopy. The work presented in this thesis makes intensive use of force spectroscopy as we measure force-distance curves at solid-liquid interfaces.

Essentially, an AFM system consists of five modules: the cantilever, the actuators (including X-Y-Z piezo and the excitation unit for the cantilever), the detector, the signal processor and the controller (see Fig. 2.2). 


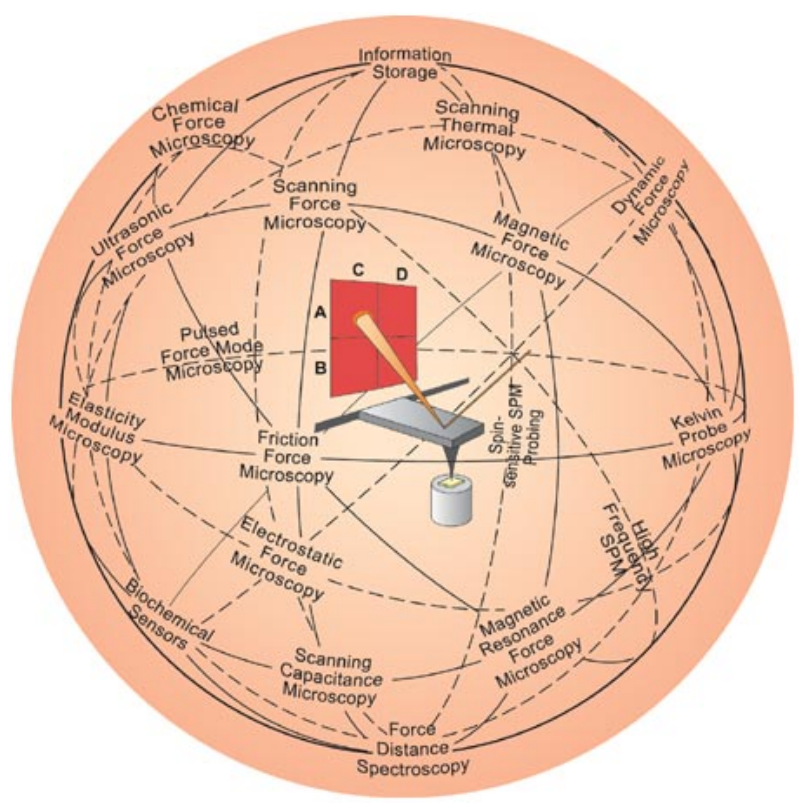

Figure 2.1: Microscopy and Spectroscopy based on AFM (adapted from [2]).

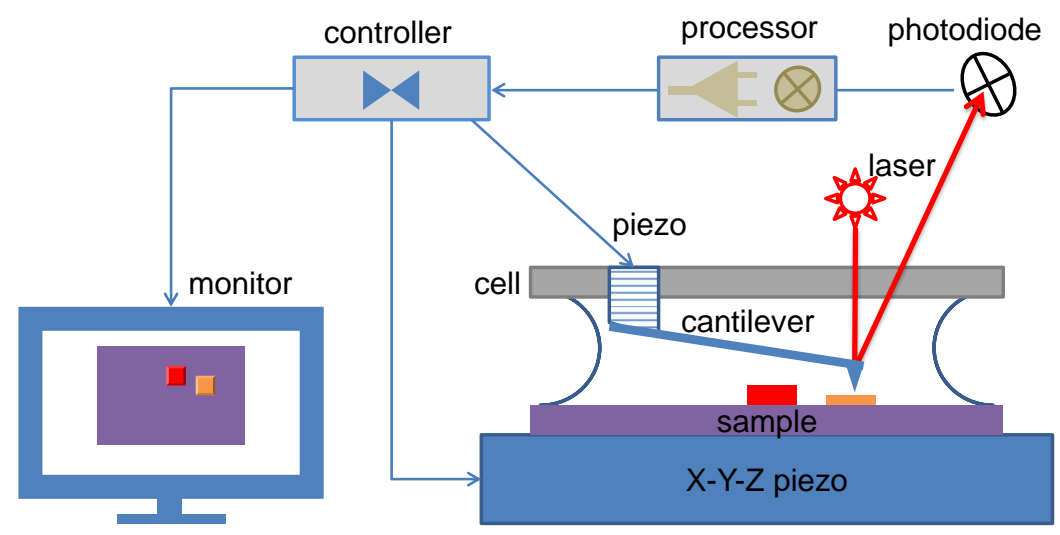

Figure 2.2: A schematic presentation of an AFM. 


\subsubsection{Static AFM force spectroscopy}

A cantilever is a thin beam with a tip on its end. It is usually made of silicon or silicon nitride. Its typical dimensions are: 50 to $200 \mu \mathrm{m}$ long, 20 to $30 \mu \mathrm{m}$ wide, 0.5 to $2 \mu \mathrm{m}$ thick. The height of a tip is approximately $15 \mu \mathrm{m}$ and the radius of curvature of its apex ranges from $1 \mathrm{~nm}$ for sharp tips to $10 \mu \mathrm{m}$ for colloidal probes. The bending stiffness of a homogeneous cantilever with a rectangular cross-section can be calculated from

$$
k_{c}=\frac{E W T^{3}}{4 L^{3}} .
$$

Here $E$ is the elastic modulus, $W$ is the width, $T$ is the height, and $L$ is the length. However, in practice, the stiffness of a cantilever is often not obtained through Eq. (2.1), because manufacturer's data have a large deviations. Alternatively, the stiffness is determined from its thermal fluctuations [6]. In principle, the stiffness is

$$
k_{c}=\frac{k_{B} T}{<z^{2}>}
$$

where $k_{B} T$ represents thermal energy and $\left\langle z^{2}\right\rangle$ is the mean square displacement due to thermal fluctuation.

In close proximity of a sample, a cantilever deflects, due to mechanical interactions with the sample, for instance electrostatic attraction. The deflection of the cantilever is detected by an interferometer or the reflection of a laser beam from the cantilever's end. The latter approach is more widely applied in commercial instruments, including the ones used for the work presented in this thesis. When the cantilever bends, the position of the laser spot on the photo-diode changes. Through a calibration procedure, the electrical signal from the photo-diode is translated to the tip displacement (see Fig. 2.3 for a sketch).

The cantilever's tip approaches or retracts from the sample surface at a controllable rate through the Z-piezo. Both the cantilever deflection and the variations of the Z-piezo position are recorded (see Fig. 2.4). The force is obtained by multiplying the displacement of the cantilever with its stiffness.

$$
F=k_{c} z .
$$

The deflection is linear with the Z-piezo position, that is, the slope is -1 when the cantilever tip is in hard contact with the surface (see Fig. 2.4). The 


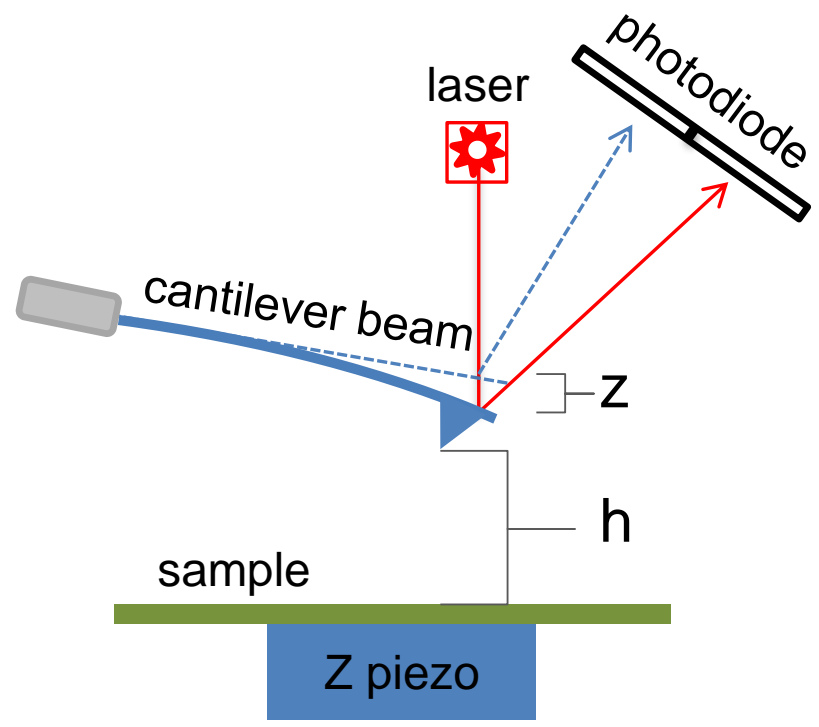

Figure 2.3: A schematic representation of static AFM force spectroscopy.

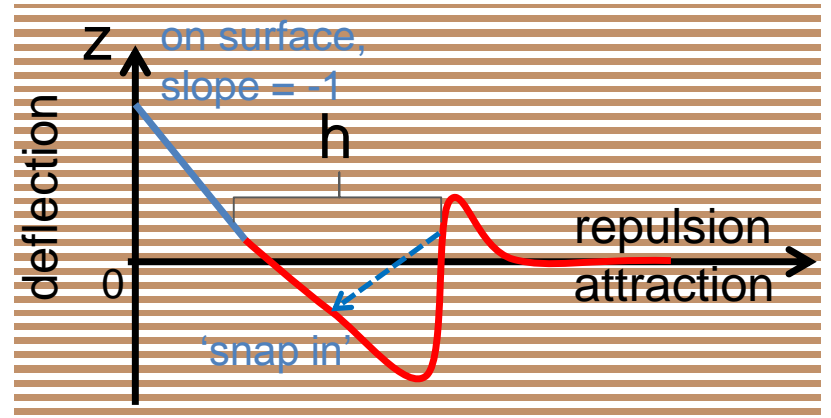

\section{position of $Z$ piezo}

Figure 2.4: An example of a deflection-piezo position curve. When the tip is in hard contact with the surface, the slope of the curve is kept at -1 . At the separation 'h' $\left(\frac{d F}{d h}>k_{c}\right)$, the instability occurs. 
separation between the tip and the substrate along such a curve is determined from the horizontal distance between the straight part (its extrapolation) and each point on the curve (see Fig. 2.4 for an example). For stable operation, the probed force gradient should be smaller than the stiffness of the cantilever: $\frac{d F}{d h}<k_{c}$. Otherwise, during approaching, the tip snaps in (see Fig. 2.4). Hence, stiffer cantilevers have to be used to prevent this instability and to obtain continuous force-distance curves. However, the sensitivity goes down when a stiffer cantilever is used, because the deflection of the cantilever is less for the same force. To improve the sensitivity, dynamic force spectroscopy is applied.

\subsubsection{Dynamic AFM force spectroscopy}

In dynamic force spectroscopy, the dynamics of a cantilever are often modeled as a simple harmonic oscillator (SHO) [7]. In most cases, the model is sufficiently accurate with exception of very viscous ambient [8] (see Chapter 4 ). The cantilever is driven to oscillate sinusoidally. Several methods for actuation are available, depending on the manufacturer: piezo excitation, magnetic excitation, or more recently, photo-thermal excitation. In piezo excitation mode, a cantilever is acoustically driven by a piezo stack attached to its base. Magnetic excitation is applied through a magnetic coating on top of the cantilever or a glued magnetic bead on the cantilever's end, above which a coil is mounted to generate an alternating magnetic field $[9,10]$. Photothermal excitation is based on the bimetallic effect $[11,12]$. A cantilever with a coated layer on top (usually aluminum or gold on silicon) is actuated by an intensity-modulated laser (usually blue laser of $\sim 1 \mathrm{~mW}$ ) focused on its base. The wavelength is different from the (red) laser for detection, so that interference is avoided. The adsorbed heat (maximum temperature increment is a few degrees) leads to transverse bending because of a difference in the thermal expansion coefficients. The different excitation methods are schematically represented in Fig. 2.5.

When the cantilever is actively driven, we acquire not only the DC deflection of the cantilever but also its amplitude, phase, and frequency. The amplitude is often set in the range from 0.1 to $10 \mathrm{~nm}$. The dynamics of a cantilever in liquid is dependent on the drive scheme [13]. Here we only introduce the simplest case. The governing equation is [7]

$$
m^{*} \ddot{z}+\gamma_{c} \dot{z}+k_{c} z=F_{\text {drive }},
$$

where $m^{*}$ is the effective mass of the cantilever, $z$ is the displacement, $\gamma_{c}$ is 
(a)

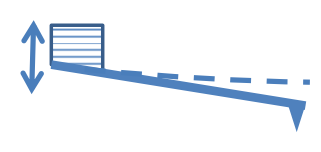

(b)

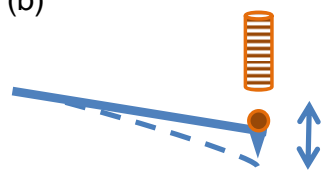

(c)

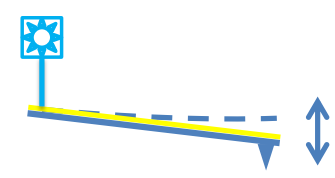

Figure 2.5: Three commonly used excitation methods. (a) A cantilever is driven by shaking its base with a piezo. (b) A cantilever is driven by a magnetic bead exposed to $\mathrm{AC}$ electromagnetic fields. (c) A cantilever with gold/alumina coating is thermally bended by periodic laser exposure.

the damping of the cantilever beam. The resonance frequency $\omega_{o}$ and the quality factor $Q$ are defined as

$$
\omega_{o}=\sqrt{k_{c} / m^{*}}, \text { and } Q=m^{*} \omega_{o} / \gamma_{c} .
$$

The effective mass takes into account the hydrodynamic loading from the surrounding liquid, which is often larger than the intrinsic mass of the cantilever. The resonance frequency in liquid is typically between $10 \mathrm{kHz}$ and $150 \mathrm{kHz}$. The quality factor in liquid is typically $<10$.

Suppose that $F_{\text {drive }}=A_{\text {drive }} k_{c} e^{j \omega_{d} t}$, the steady state of the cantilever displacement is expressed as

$$
z=A e^{j\left(\omega_{d} t+\phi\right)},
$$

where $A$ is the amplitude, $\phi$ is the phase with respect to the driving signal, $\omega_{d}$ is the drive frequency. From Eq. (2.4), we get the transfer function of an SHO,

$$
\begin{aligned}
\frac{z}{F_{\text {drive }}}=\frac{A e^{j \phi}}{A_{\text {drive }} k_{c}} & =\frac{1}{-m^{*} \omega_{d}^{2}+j \gamma_{c} \omega_{d}+k_{c}} \\
& =\frac{1}{k_{c}} \frac{1-\left(\omega_{d} / \omega_{o}\right)^{2}-j \omega_{d} /\left(\omega_{o} Q\right)}{\left(1-\left(\omega_{d} / \omega_{o}\right)^{2}\right)^{2}+\omega_{d}^{2} /\left(Q \omega_{o}\right)^{2}} .
\end{aligned}
$$

At resonance, i.e. $\omega_{d}=\omega_{o}$, the phase $\phi$ is $-90^{\circ}$ and $A=A_{d r i v e} Q$. When it is driven off resonance, the phase is described by

$$
\tan \phi=\frac{\omega_{o} \omega_{d} / Q}{\omega_{d}^{2}-\omega_{o}^{2}}
$$



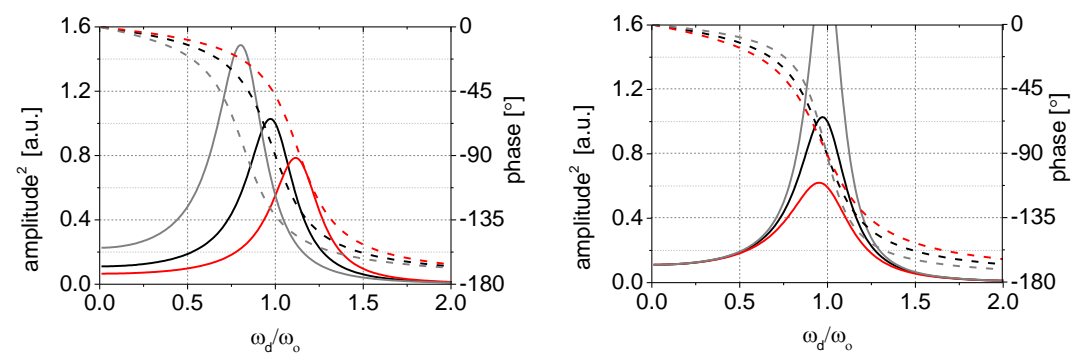

Figure 2.6: The frequency response of a simple harmonic oscillator (SHO) and the effect of interactions. The amplitude (solid line) and phase (dashed line) for an unperturbed SHO $(Q=3)$ is in black. When a conservative force is exerted, the responses are plooted in the left (gray, $k_{i n t}=-0.3 k_{c}$; red, $\left.k_{i n t}=0.3 k_{c}\right)$. When the interaction is dissipative, the responses are plotted in the right (gray, $\gamma_{i n t}=-0.3 \gamma_{c}$; red, $\gamma_{i n t}=0.3 \gamma_{c}$ ).

In Fig. 2.6, the amplitude and phase response of an SHO is shown. The transfer function, Eq. (2.7), relates the measured quantities, the amplitude and phase of the cantilever in response to the driving signal, to the physical quantities of interest, namely, the interaction stiffness and the interaction damping (see the next section). For any quantitative force measurement in $\mathrm{AFM}$, it is therefore essential that the transfer function is well-defined and properly measured in the experiments. An important part of this thesis (and the general AFM spectroscopy literature in liquids) is therefore devoted to the development of reliable procedures to measure the transfer function.

\section{How to obtain the interaction forces from the responses of the cantilever}

Force spectroscopy is a technique for measuring distance-dependent forces. During the measurement, we monitor the response of a vibrating cantilever when it interacts with a surface at different separations. In this case, the governing equation is rewritten as

$$
m^{*} \ddot{z}+\gamma_{c} \dot{z}+k_{c} z=F_{\text {drive }}+F_{t s}(h+z, \dot{z}),
$$

where $\mathrm{d}$ is tip-sample separation in equilibrium, $F_{t s}$ is tip-sample interaction force accounting for both conservative and dissipative contributions. 


\section{Small amplitude}

Provided that the amplitude $A$ is sufficiently small, the tip-sample interaction force may be linearized as

$$
F_{t s}(h+z, \dot{z})=F_{t s}(h, 0)-k_{i n t} z-\gamma_{i n t} \dot{z},
$$

where $k_{\text {int }}$ is the interaction stiffness equal to $-\partial F_{t s} / \partial h, \gamma_{\text {int }}$ is the interaction damping coefficient $\left(\partial F_{t s} / \partial \dot{z}\right)$, while $F_{t s}(h, 0)$ is the equilibrium force. From Eqs. (2.5) and (2.9), it is clear that we need to know $\omega_{o}, Q$ and $k_{c}$ before we can determine the tip-sample interaction $F_{t s}$. All of them are determined through a procedure, which is called thermal calibration. In Chapter 3, we will give more details. For the time being, we take them as known parameters. Then the force inversion equations are

$$
k_{i n t}=k_{c}\left[-1+\left(\frac{\omega_{d}}{\omega_{o}}\right)^{2}+\frac{A_{d r i v e} \cos \phi}{A}\right]
$$

and

$$
\gamma_{i n t}=\frac{-A_{d r i v e} k_{c} \sin \phi}{A \omega_{d}}-\gamma_{c}
$$

where $A_{\text {drive }}$ may be determined from Eq. (2.9) with the measured $A_{\infty}$ and $\phi_{\infty}$ in the case $F_{t s}=0$, i.e. amplitude and phase are measured far from the surface.

If we keep the drive force $F_{\text {drive }}$ in Eq. (2.9) fixed along an approach curve, the operation mode is referred to as amplitude modulation (AM). If the drive frequency is not close to the resonance of the cantilever, i.e. $\left|\omega_{d} / \omega_{o}-1\right|>0.1 \sim 0.2$, it is called off-resonance. Otherwise, it is called nearresonance. The counterpart of AM is frequency modulation (FM) $[14,15]$. In FM, the phase of the cantilever oscillation is locked at $-90^{\circ}$, that is, the drive frequency $\omega_{d}$ is adjusted to match the resonance frequency of an SHO in the presence of perturbation. The frequency shift $\Delta \omega_{o}$ is the difference between $\omega_{d}$ and $\omega_{o}\left(\Delta \omega_{o}=\omega_{d}-\omega_{o}\right)$. Then, the corresponding force inversion equations are

$$
k_{i n t}=k_{c}\left[\left(\frac{\omega_{d}}{\omega_{o}}\right)^{2}-1\right]
$$

and

$$
\gamma_{\text {int }}=\frac{A_{d r i v e} k_{c}}{A \omega_{d}}-\gamma_{c}
$$

If $k_{\text {int }}<<k_{c}$, Eq. (2.13) may be rewritten as $k_{i n t}=2 k_{c} \Delta \omega_{o} / \omega_{o}$.

In FM, the amplitude of the cantilever oscillation can be fixed by adjusting the drive force magnitude $F_{\text {drive }}$. The mode is called constant amplitude 
FM (CA-FM) [14]. Otherwise the magnitude of $F_{\text {drive }}$ is fixed, then it is called constant excitation (CE-FM). CE-FM is less common than CA-FM, because for CA-AFM, force inversion equations for arbitrary amplitude are available $[16,17]$. Compared to AM, the CA-FM is more convenient in case the characteristic length of the interaction force is so small that the amplitude of the cantilever oscillation does not justify the linearization in Eq. (2.10). Actually, the CA-FM is prevailing in the application of very high vacuum AFM, where the interaction force is often modulated in the angstrom scale. Recently, CA-FM has also been applied in liquid [4].

The advantage of AM-AFM over FM-AFM is that there is no feedback loop on phase or amplitude and thereby it is simpler and more robust. The strength of FM-AFM is that the conservative force and dissipation are measured in a decoupled manner. As shown in Eqs. (2.13) and (2.14), the force gradient can be evaluated directly from the frequency shift, and the dissipation can be inferred from the drive amplitude $A_{\text {drive }}$ in CA-FM, or the amplitude $A$ in CE-FM. It not is applicable in AM-AFM, as shown in Eqs. (2.11) and (2.12).

So far, we offered a brief introduction on how to measure interaction forces. However, in practice, the application of AFM force spectroscopy in liquid meets several challenges, as discussed in Section 2.2.

\subsection{Challenges with AFM force spectroscopy in liquid}

First and foremost, the cantilever dynamics in viscous liquid is not understood as well as in vacuum. It is well established that the approximation of the cantilever dynamics as an SHO is sufficient for its application in air and vacuum $(Q>100)$ [7]. However, in liquid, the quality factor goes down dramatically $(Q<10)$ because of the hydrodynamic loading. The accuracy of SHO approximation decreases [8] with decreasing $Q$. Also, the excitation efficiency decreases with the quality factor $Q$. Under liquid, the base motion needs to be taken into account in piezo excitation mode $[18,19]$. Moreover, it is recently shown that in piezo excitation mode the cantilever is not only directly driven by the piezo, but also via the piezo excited surrounding liquid [20] (see Fig. 2.7). The fluid-mediated excitation is comparable to the direct excitation of the piezo. As a result, the dynamics of a pizeo-excited cantilever in liquid become even more complex because the fluid mediated driving effect asks for a continuous beam description of the cantilever [20]. 


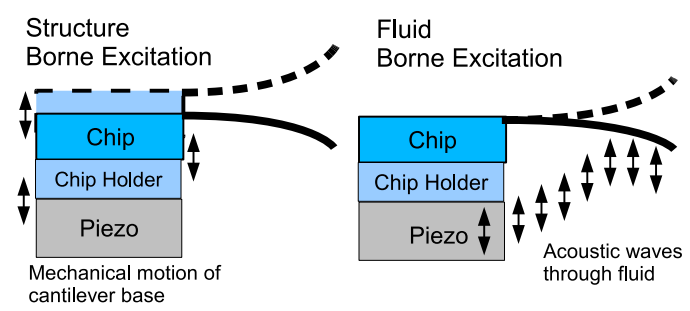

Figure 2.7: A cantilever is excited directly by a vibrating piezo in liquid (structure borne excitation, left) and indirectly by an acoustic wave in liquid (fluid borne excitation, right), generated by the piezo. (adapted from [20])

Secondly, the damping under a tip is small in most cases, compared to the hydrodynamic damping on the cantilever beam. Hence, it is sensitive to errors in modeling during force inversion. For instance, in the case of near-resonance AM, a minor error in phase leads to considerable coupling between conservative and dissipative parts [21]. The FM analysis requires a strict SHO description of the dynamics. However, it is known that the cantilever-drive unit combination does not behave as an SHO, although the cantilever itself may resemble an SHO. It is because the response of the drive unit is usually frequency dependent. In particular, the most commonly used piezo excitation has a spurious transfer function, which together with the cell geometry, results in the notorious 'forest of peaks' [22] (see Fig. 2.8). It means that in FM the detected variables are not purely a function of the tip-sample interaction, but they are affected by the frequency-dependent response of the drive unit. It may give rise to artificial dissipation [23, 24]. Much effort has been dedicated to solving the problems of piezo excitation [20, 24-28]. Recently, photothermal excitation, as a promising technique, became commercially available [29]. It does not suffer from 'forest of peaks' in transfer function and is stable over hours. In Chapter 5, we present measurements performed with photothermal excitation.

Thirdly, there are no common protocols for the AFM force measurement. Different laboratories employ different techniques. The cantilevers are actuated differently, including piezo excitation, magnetic excitation and photothermal excitation, as mentioned above. There are various operation modes for AFM force spectroscopy, including near-resonance AM, off-resonance AM, CA-FM, CE-FM and others. Among the studies in confined liquids, there are only a few measurements conducted with the same technique (see Sec- 


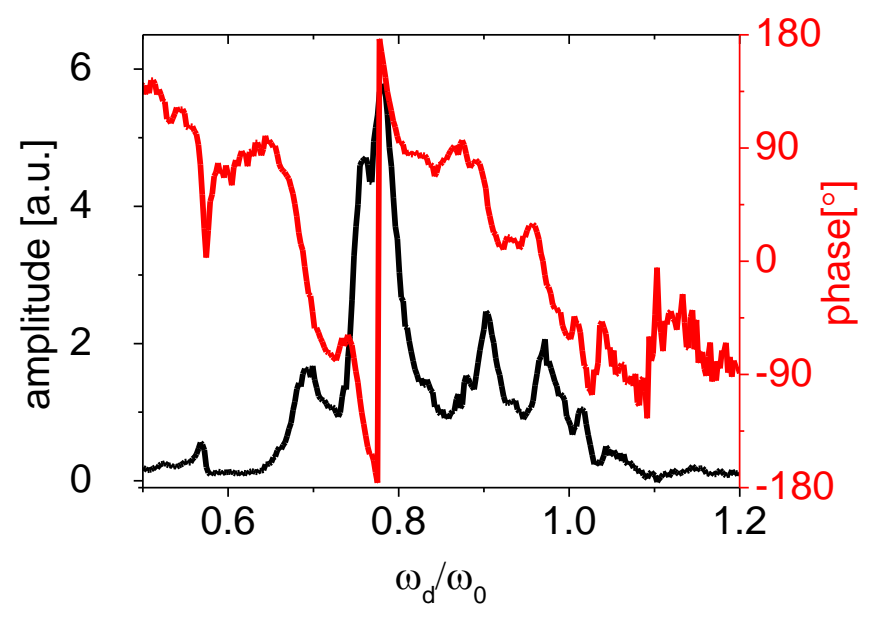

Figure 2.8: The frequency response of a cantilever driven by a piezo in liquid.

tion 2.3.2). Also the accuracy of each technique is not always clear. All these techniques are hardly validated. Along the same line, the geometry of a tip is usually not well defined, which is essential for quantitative analysis. In some cases, discrepancies in results are attributed to different types of tips used $[25,30]$. In sum, dynamic AFM force spectroscopy in liquid is non-trivial and the theories and techniques are still under development

\subsection{Thin liquid film mediated forces and interpretations}

Using AFM force spectroscopy in liquid, one probe both the conservative force and the viscous dissipation between the tip and the sample. In the continuum regime, classical theories are available for the interaction force, including hydrodynamic dissipation and the DLVO (named after the pioneers Derjaguin, Landau, Verwey and Overbeek) theory. However, when the film is a few molecular layers thick, the continuum theories often fail and the discrete nature of the solvent and solutes comes into play. 


\subsubsection{Conservative forces}

Direct nanoscopic force spectroscopy has been employed to measure the DLVO force $[5,31,32]$. Good agreement between measurement and theory is often achieved. In an AFM measurement, the sensing tip is usually made of silicon and its surface is covered by a few nanometer native silica. In water, the tip becomes charged, due to the deprotonation of the silanol groups ( $\mathrm{Si}$ $\mathrm{O}-\mathrm{H})$ [33]. Hence, when analyzing the measured force distance curves one should take into account the resulting electric interactions. To deal with this, we first introduce the theory for the DLVO force and then present how to solve the Poisson-Boltzmann equation with proper boundary conditions.

\section{DLVO force}

According to the well-established DLVO theory, the disjoining pressure between two objects is the algebraic sum of the van der Waals component and the double layer component [34],

$$
\Pi=\Pi_{v d w}+\Pi_{d l} .
$$

For two semi-infinite parallel plates, the van der Waals part is

$$
\Pi_{v d w}=-\frac{A}{6 \pi h^{3}},
$$

where $h$ is the thickness of the medium, $A$ is the Hamaker constant. The Hamaker constant in a liquid medium is usually in the range between $10^{-21}$ and $10^{-20} \mathrm{~J}$. The double layer pressure has two components-the osmotic pressure and the Maxwell stress,

$$
\Pi_{d l}=k_{B} T \sum_{i}\left[n_{i}-n_{i(\infty)}\right]-\frac{1}{2} \varepsilon \varepsilon_{0}(\nabla \phi)^{2} .
$$

The tip is often not flat, but has a finite curvature. Given $h<<R_{\text {tip }}$, the shape of a spherical tip can be assumed to be parabolic. Using Derjaguin approximation, the conservative force can be obtained by integrating the pressure over the tip surface,

$$
F=\int_{0}^{\infty}\left\{\Pi_{\mathrm{dl}}(h)+\Pi_{v d W}(h)\right\} 2 \pi r d r
$$

where $h=h_{0}+r^{2} /\left(2 R_{\text {tip }}\right)$ represents the tip-substrate distance $h_{0}$ and the parabolic approximation $r^{2} /\left(2 R_{\text {tip }}\right)$ for the shape of the tip. Hence, the 
corresponding force gradient or interaction stiffness $k_{\text {int }}=-\partial F_{\text {int }} / \partial h_{0}$ will be given by

$$
k_{\text {int }}(h)=2 \pi R_{\text {tip }}\left\{\Pi_{\mathrm{dl}}(h)+\Pi_{v d W}(h)\right\}
$$

The knowledge of the charge distribution in the gap is required, as shown in Eq. (2.17), to compare our data to theory.

Electric double layers The charge distribution near a charged surface is called electric double layer (EDL) [34]. Various models are proposed to describe the EDL, such as Helmholtz model, Gouy-Chapman model, and Stern model. In the Stern model, the EDL is 'somewhat artificially' divided into two parts [34]. The inner part is a Stern layer and the outer part is a diffuse layer (see Fig. 2.9). The Stern layer is a condensed structure of specifically and non-specifically adsorbed species on the surface. Its thickness is often assumed to be $<1 \mathrm{~nm}$. On top of the Stern layer, there is a diffuse layer, which results from the balance between coulomb attraction and thermal diffusion. We call the interface between the Stern layer and the diffuse layer the Stern plane. The characteristic dimension of the diffuse layer thickness is known as the Debye length, which is determined by the concentrations of the electrolyte. The ionic strength is $I_{\infty}=\frac{1}{2} \sum_{i} Z_{i}^{2} n_{i(\infty)}$, where $i$ denotes each ionic species, $n_{i(\infty)}$ is its bulk concentration and $Z_{i}$ is its valency. The reciprocal of the Debye length is

$$
\kappa=\sqrt{\frac{2 I_{\infty} e^{2}}{\varepsilon \varepsilon_{0} k_{B} T}} .
$$

The charge density in the diffuse layer decreases to zero when moving from the interface to the bulk, i.e. $\sum_{i} Z_{i} n_{i(\infty)}=0$. The density of the ionic species obeys the Boltzmann relation:

$$
n_{i}(\phi)=n_{i(\infty)} \exp \left(\frac{-e Z_{i} \phi}{k_{B} T}\right),
$$

where $\phi$ is the local potential. The charge-potential relation is governed by the Poisson equation:

$$
\nabla^{2} \phi=\frac{-\rho}{\varepsilon \varepsilon_{0}},
$$

where $\rho=\sum_{i} e Z_{i} n_{i}(\phi)$ is the charge density. Unifying Eqs. (2.20) and (2.21), we obtain the well-known Poisson-Boltzmann (P-B) equation:

$$
\nabla^{2} \phi=\frac{-e}{\varepsilon \varepsilon_{0}} \sum_{i} Z_{i} n_{i(\infty)} \exp \left(\frac{-e Z_{i} \phi}{k_{B} T}\right),
$$


(a)

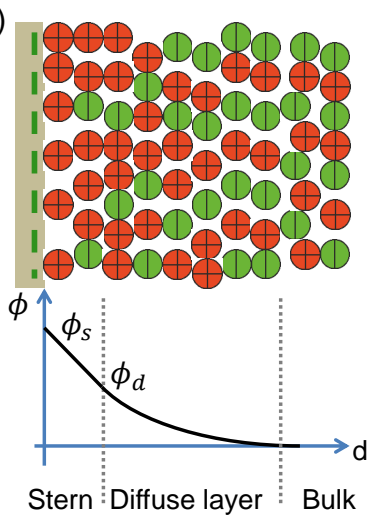

(b)

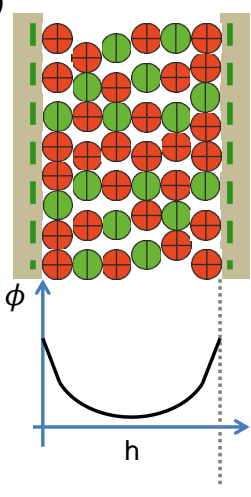

Figure 2.9: (a) A schematic representation of the electrical double layer (EDL) structure on the surface of an isolated plane. It is comprised of a compact stern layer and a diffuse layer. At large distances, $h \rightarrow \infty$, the potential and the charge density drop to zero. (b) The EDL structure between two adjacent plates when the diffuse layers are overlapping.

\section{Boundary conditions for the P-B equation:}

Charge regulation To solve the $\mathrm{PB}$ equation we need expressions for the surface charge or surface potential. There are three types of boundary conditions under consideration: constant charge (CC), constant potential (CP), and charge regulation (CR). The $\mathrm{CC}$ and $\mathrm{CP}$ boundary conditions are often used [31], for instance, when the surface potential of a conductive surface is fixed. The CR boundary condition is more realistic for force spectroscopy, such as to analyze force measurements between an AFM tip and a sample surface in presence of electrolytes. It is more realistic because the presence of the tip regulates the distribution of ions in the film between tip and substrate and consequently the charge on the sample surface (and the tip). Physically, the diffuse layers from the tip and the substrate are overlapping, then the concentrations of the ions are changed, and eventually the surface charge is changed. Therefore, we need to formulate a boundary such that it accounts for the fact that the charge density of the surface (and the tip) changes with the separation between them. The CR boundary condition is constructed from surface complexation models [32,35-37].

Surface complexation models As an example, we consider two surface reactions: deprontonation and adsorption of cations. The deprotonation of 
surface groups is common, especially metal oxides immersed in water,

$$
\sim S H \rightleftharpoons \sim S^{-}+H^{+}
$$

with an equilibrium constant,

$$
K_{H}=\frac{\left\{S^{-}\right\}\left[H^{+}\right]_{s}}{\{S H\}},
$$

where $\left\{S^{-}\right\}(\{S H\})$ is the site density of the deprotonated (undeprotonated) surface group. The deprotonated surface group may be taken by the counterions in the solution to form a surface complex. It results in another surface reaction:

$$
\sim S C^{Z c-1} \rightleftharpoons \sim S^{-}+C^{Z c},
$$

with an equilibrium constant,

$$
K_{C}=\frac{\left\{S^{-}\right\}\left[C^{Z c}\right]_{d}}{\left\{S C^{Z c-1}\right\}},
$$

where $Z c$ is the valency of the cation $C$. The total site density $\Gamma$ is conserved:

$$
\Gamma=\left\{S^{-}\right\}+\{S H\}+\left\{S C^{Z c-1}\right\} .
$$

Unifying Eqs. (2.23), (2.25) and (2.27), we obtain a matrix,

$$
\left(\begin{array}{ccc}
1 & 1 & 1 \\
-\left[H^{+}\right]_{s} & K_{H} & 0 \\
-\left[C^{Z c}\right]_{d} & 0 & K_{C}
\end{array}\right)\left(\begin{array}{c}
\left\{S^{-}\right\} \\
\{S H\} \\
\left\{S^{Z c-1}\right\}
\end{array}\right)=\left(\begin{array}{c}
\Gamma \\
0 \\
0
\end{array}\right)
$$

Note that the concentrations of the ions $\left(\left[\mathrm{H}^{+}\right]_{s}\right.$ and $\left.\left[\mathrm{C}^{Z c}\right]_{d}\right)$ are the values at the surface and the Stern plane, respectively, which are different from the bulk. They follow the Boltzmann relation:

$$
\begin{gathered}
{\left[H^{+}\right]_{s}=\left[H^{+}\right]_{\infty} \exp \left(\frac{-e \phi_{s}}{k_{B} T}\right)} \\
{\left[C^{Z c}\right]_{d}=\left[C^{Z c}\right]_{\infty} \exp \left(\frac{-e Z c \phi_{d}}{k_{B} T}\right)}
\end{gathered}
$$

Here $\phi_{s}$ is the surface potential. The surface potential is related to the potential at the Stern plane by considering the capacitance of the Stern layer, 
$C_{s}=\frac{\sigma}{\phi_{s}-\phi_{d}}=\frac{-e\left\{S^{-}\right\}}{\phi_{s}-\phi_{d}}$. Eventually, we have established the CR boundary condition:

$$
\sigma=f\left(\phi_{s} ; K_{H}, K_{C}, \Gamma,\left[H^{+}\right]_{\infty},\left[C^{Z c}\right]_{\infty}, C_{s}\right)
$$

We note that the extracted surface charge $\sigma$ is essentially the net surface charge, i.e. the opposite of the diffuse charge.

The P-B theory for describing the EDL is a mean-field approach. It does not catch the discrete nature of the solution at the molecular scale. When the liquid film is only molecularly thick, the non-DLVO forces are dominant. The non-DLVO forces include solvation forces, hydrophobic forces and steric forces [38]. The hydrophobic force is the attractive interaction between two hydrophobic surfaces in water. The steric force originates from the volume exclusion effect, existing between two rough surfaces. Each type of non-DLVO forces is a subject of interest. We confine ourselves to discussing solvation forces in the study of non-DLVO forces.

\section{Solvation forces}

The first oscillatory solvation forces were measured in an inert organic liquid between two atomically smooth macroscopic mica sheets in 1980 [39], using a surface forces apparatus (SFA) (see Fig. 2.10). It is a remarkable breakthrough in the investigation of the surface force: It is the first direct evidence that the interfacial liquid is not bulk-like, and that the solvation structure is periodic, echoing the results from computer simulations [40]. Layering is caused by excluded volume effects and comparable with the pair-probability distribution of molecules around a tagged molecule in the bulk, that is, it is not monotonic but oscillatory. When an atomically flat wall is present, the molecules are attracted to the surface and form a quasi-discrete structure [38]. Several molecular diameters way from the surface, the effect tapers off. Empirically, the oscillatory solvation force is described by [38]

$$
F=f_{0} \cos \left(\frac{2 \pi H}{\sigma}\right) \exp \left(-\frac{H}{\lambda}\right),
$$

where $f_{0}$ is the prefactor, $H$ is the thickness of the confined film, $\sigma$ is the periodicity and $\lambda$ is the decay length. The magnitude of $F$ is usually larger than the van der Waals force.

A dozen years after the first SFA measurements, the first AFM measurements on such oscillatory forces were reported [41]. One major advantage of AFM over SFA is that the samples are not limited to macroscopic (coated 


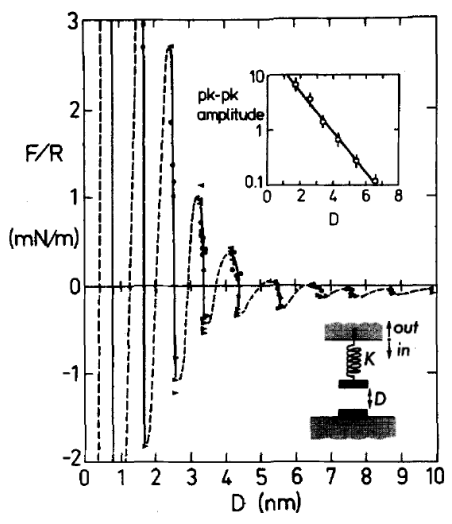

Figure 2.10: Experimental results of the force $F$ as a function separation $D$. The attract regime (dashed line) is not measurable because of instability. The inset at the bottom is a sketch of the surface force apparatus (SFA) setup. The surfaces are two cylindrically curved mica, $R \approx 1 \mathrm{~cm}$. The liquid is octamethylcyclotetrasiloxane (OMCTS, $\left.\left[\left(\mathrm{CH}_{3}\right)_{2} \mathrm{SiO}\right]_{4}\right)$. (adapted from [39]).

(a)

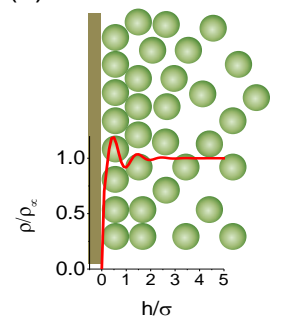

(b)

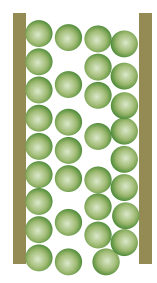

(c)

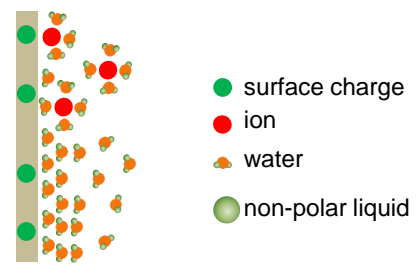

Figure 2.11: The liquid structure near a smooth wall. The liquid has a quasi-discrete structure and its density is oscillatory decaying as a function of distance from the wall. (a) The interface between a single wall and nonpolar liquid. (b) Non-polar liquid confined between two walls. (c) Hydrated ions and layered water near a charged wall. 
or not) mica sheets with a typical size $R \approx 1 \mathrm{~cm}$. It enables us to study surface specificity of the solvation forces. Another advantage is that tip sizes are available from nanoscale $(R<10 \mathrm{~nm})$ to microscale $(R>1 \mu \mathrm{m})$. It enables us to probe forces under different resolution. The tip, as an asperity of certain roughness resembles more the realistic case in applications than the atomically flat mica.

Hydration force The hydration force is a term dedicated to the solvation force in water. The hydration forces occur when two hydrophilic surfaces are placed in close proximity. The solvation structure of water near a (charged) surface is much more complicated than the non-polar liquids (see Fig. 2.11). The empirical evidence shows that the hydration forces are short-ranged, negligible at separations $>4 \mathrm{~nm}$. Two types of hydration are distinguished according to its origin: the primary hydration and the secondary hydration. The primary hydration originates from the inherently adsorbed water on the surface [42]. The secondary hydration originates from the hydration of solutes adsorbed near the surface [42]. The hydration force is arguably one of the core problems in physical chemistry [42].

To get clearer picture of the hydration forces, the surface specificity, ion specificity and ion concentration dependence of the hydration forces have been explored. Using SFA [43,44], it is found that the strength of the hydration force between two mica surfaces is correlated with the hydration shell of adsorbed cations, that is, more hydrated ions give rise to stronger hydration forces. For silica, it is the opposite. On silica coated mica, the strength and range of the (monotonic) hydration forces decrease with increasing degree of hydration of the cations in the background solutions. For monovalent ions, the hydration force increases in the following order: $\mathrm{Li}^{+}<\mathrm{Na}^{+}<\mathrm{K}^{+}<\mathrm{Cs}^{+}$[45]. AFM measurements with a nanoscopic sharp silica tip on a mica substrate suggest the hydration forces increase with the ionic strength and the valency of the cation [46].

The experimentally observed hydration force is not always oscillatory, as described in Eq. (2.32), but can be monotonic. One appealing explanation for this monotonic behavior is that the surface roughness smears out the original oscillations due to the large rough contact area. It is plausible in the sense that the diameter of a water molecule is only around $0.25 \mathrm{~nm}$. In a laboratory environment, one can only prepare a few surfaces that are atomically smooth, including cleaved graphite, mica.

From experimental perspective, it is not trivial to discriminate the hydration force from other forces. Practically, hydration forces are mostly obtained by subtraction of the DLVO force from the total force [43-46]. The DLVO force at the short range is extrapolated from the forces measured at the large 
separations, where the hydration force is negligible. It is vulnerable to criticism on the ground that the extrapolation may not be justified, because, as we know, the DLVO theory fails when the film thickness between two surfaces is of the order of molecular size. Moreover, the DLVO force and hydration force may not be additive.

\subsubsection{Dynamic properties of molecularly thin films}

It is unanimously recognized that the structure of interfacial liquid is different from the bulk. However, it is still controversial whether and to what extent the structural difference leads to peculiar dynamic properties, for instance viscosity enhancement. It is mainly measured in two alternative ways: the squeeze-out force for an approaching surface or the shear force for a laterally moving surface over the confined liquid on a surface (the squeeze flow and the Couette flow, see Fig. 2.12).

In most cases, the size of the plate is much larger than the thickness of the confined film, $R / h>>1$. For the squeeze flow, the pressure on the plate follows the Reynolds equation, using the lubrication approximation for axisymmetric systems with "no slip" boundary conditions $[47,48]$,

$$
\frac{1}{r} \frac{\partial}{\partial r}\left(\frac{r h^{3}}{\eta} \frac{\partial p}{\partial r}\right)=-12 v
$$

where $v$ is the approach rate, $h$ is the thin film thickness, and $\eta$ is the viscosity. The squeeze force is the integral of the pressure over the plate surface. For a flat round disk, the damping coefficient is

$$
\gamma=\frac{\partial F}{\partial v}=\eta \frac{3 \pi R^{4}}{2 h^{3}}
$$

In the AFM measurements, the tip often has a finite curvature. In the case of a sphere, Eq. (2.34) should be rewritten as

$$
\gamma=\eta \frac{6 \pi R^{2}}{h}
$$

Here $R$ is the radius of the approaching spherical tip. In the case of Couette flow, the coefficient of friction is

$$
\gamma=\eta \frac{\pi R^{2}}{h}
$$


(a)

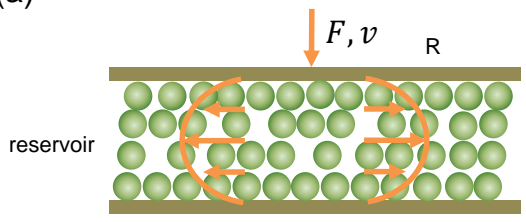

(b)

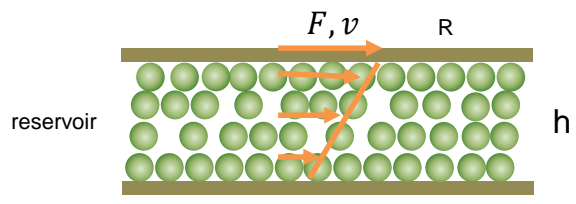

Figure 2.12: Two kinds of measurement methods of the viscous dissipation in confined liquids. (a) The squeeze flow. (b) The Couette flow. The thickness of the confined film and the radius of the plate are denoted as $h$ and $R$.

In the past two decades, controversial results were reported on the dynamic properties of confined liquids, such as viscosity and relaxation time [49]. With SFA and AFM, various liquids are investigated in different laboratories. First, we review the experimental results on octamethylcyclotetrasiloxane (OMCTS, $\left.\left[\left(\mathrm{CH}_{3}\right)_{2} \mathrm{SiO}\right]_{4}\right)$, which is most widely used as 'model liquid'.

Analyzing SFA data, Klein reported a confinement-induced liquid-solid transition $[50,51]$. The transition is reversible and abrupt. It emerges when the confined film is less than 7 molecular diameters thick. At the transition, the mean viscosity of the confined liquids is increased by at least seven orders of magnitudes.

Yoshizawa and Israelachvili postulated that when its thickness is progressively reduced, the confined film goes through three regimes: bulk-like, mixed, and boundary ( $<4$ molecule diameters) regimes. The layering in the boundary regime shows a liquid-crystalline structure [52]. These structures can melt under shear. The solidification and shear-induced melting gives rise to a stick-slip type of friction [53] (see Fig. 2.13). On the onset of slip, the confined liquid melts again and stays in a liquid like structure with a slightly increased thickness, giving rise to dilation.

The mica substrate used in SFA measurements is conventionally cut by a hot Pt wire. In 2003, Christenson et al. confirmed that after cut the mica surface is stained by Pt particles of $\sim 20 \mathrm{~nm}$ in diameter and $\sim 2 \mathrm{~nm}$ in height $[54,55]$.

Zhu and Granick [56] reexamined the measurements and reported that the effective viscosity of the film is dependent on the approach rate: slow compression gives unresolvable viscosity (experimental error $\approx 100$ times of the bulk viscosity) while quick compression leads to enhancement by five orders of magnitude. The presence of Pt particles on the mica surface due to the cut procedure could flaw the results of previous studies $[50,52]$. Therefore, 
(a)

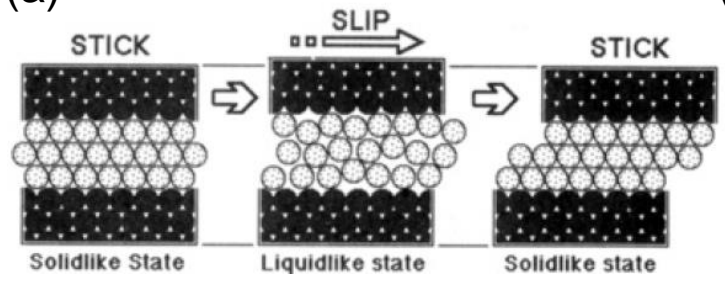

(b)

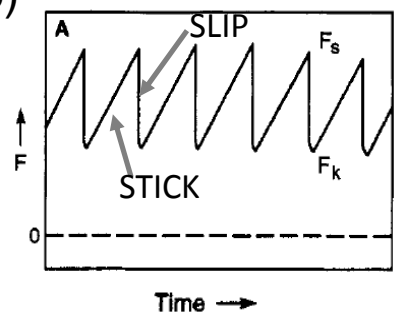

Figure 2.13: (a) Phase transistion of stick-slip friction with confined liquids (b) Friction-time traces with OMCTS sandwiched between two mica surfaces. (adapted from [52])

the measurements were repeated with substrates free of $\mathrm{Pt}$ particles [57]. Nevertheless, the stick-slip friction was not reproduced [57].

Becker and Mugele analyzed the layer-by-layer expulsion of confined liquid films, by describing the squeeze-out flow as a 2D Poiseuille flow [58] (see Fig. 2.14). Inferred from the squeeze-out front line and interlayer sliding friction, the reported viscosity is close to the bulk value, which does not imply solidification.

In 2010, Bureau reconciled the conflicting observations by studying both shear rheology and squeeze-out front measurements in the same experimental run [59]. These measurements show that the shear viscosity is enhanced by 2 orders of magnitude and the confined liquid has nonlinear flow characteristics akin to a supercooled liquid approaching its glass transition [60]. Again, the stick-slip friction is not observed. The previously reported stick-slip friction is attributed to the contamination with Pt particles on mica $[50,52,57]$. By analyzing with the front line propagation, the results are consistent with the observations reported by Becker and Mugele [58]. It is argued that the squeeze-out front line is essentially 'a defect' between the $\mathrm{n}$ layer and the $\mathrm{n}-1$ layer, and therefore its propagation is controlled by permeation, not coherent sliding of layers as Becker and Mugele proposed.

In 2015, Klein et al. reported again stick-slip friction in confined liquids [61]. More importantly, they claimed that there occurs no fluidization in the stick-slip friction process. This claim is based on the absence of dilation in their measurements. The reasoning is as follows: If fluidization (melting under shear) occurs, the density of the confined liquid film decreases. Then 


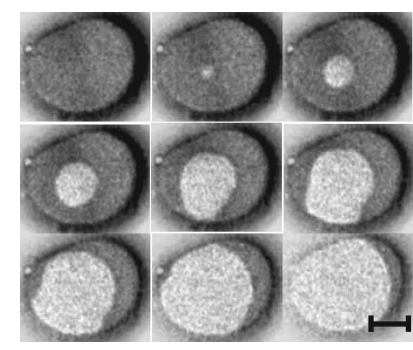

Figure 2.14: Expulsion of a confined liquid film, measured by SFA with multiple beam interferometry and video microscopy. The contact zone in gray is the initial state. The edge of the bright part is the boundary between $\mathrm{n}$ layer (dark) and n-1 layer (bright), i.e. the squeeze-out front line. Series of images with time interval $0.3 \mathrm{sec}$. Scale bar $25 \mu \mathrm{m}$. (adapted from [58])

the thickness of the film should increase, which is not observed within their experimental resolution $(100 \mathrm{pm})$. The work is criticized by Isrealachavili et al. and Granick et al. in terms of data interpretation and experimental resolution. Isrealachavili et al. point out that melting of fluid does not necessarily involve expansion [62]. Granick et al. argue that the argument by Klein et al. is contradictory to their previous findings while Granick et al. obtained superior experimental resolution in thickness [63-65]. In their rebuttal, Klein et al. argue that the work from Granick's lab may be affected by contamination of $\mathrm{Pt}$ particles and that 'dilation on melting is the rule' [66].

Using SFA, one is not able to measure in the attraction regime where the instability ('snap in') occurs (see Figs. 2.4 and 2.10). Therefore, the force distance curves from SFA are often not continuous. Recently, AFM has also been used to probe the properties of confined liquid films. In AFM, it is able to fulfill the condition for stability by using stiffer cantilevers and smaller tips. Therefore, one can probe the visco-elasticity of confined films as a function of the continuously controllable spacing between the two surfaces. However, the optical information is often lost, from which SFA offers film thickness and front line propagation.

Piezo excitation for the cantilever is most commonly used in AFM under liquid because of its ease of use and low cost [19,20,26-28,67-71]. It is known that the piezo excitation in liquid is problematic, as mentioned in Section 2.2. Nevertheless, we still include the key findings with piezo excitation in the review.

Using near-resonance AFM, Maali et al. observed a modulated dissipa- 
tion: The oscillatory stiffness of the film as a function of thickness is accompanied by an oscillatory squeezing-out damping [67]. Using off-resonance AM, Hoffmann et al. $[68,69]$ also reported that the dissipation is oscillatory. More importantly, it is dependent on the compression rate. Using a visco-elastic Maxwell model, the authors converted the stiffness and damping coefficient of the confined film to a relaxation time,

$$
t_{R}=\frac{k}{\gamma \omega_{d}^{2}}
$$

where $\omega_{d}$ is the oscillation frequency of the AFM tip.

Above a critical compression rate, the confined film is jammed and has a large relaxation time, like solids (see Fig. 2.15). The authors also offered an explanation for the oscillatory behavior of the dissipation: When the gap height is commensurate with molecular size, the liquid molecules are in the ordered state and thereby the film responds more elastic (stiffer but less viscous) like solids under strain. In the incommensurate case, the film is in the disorder state and softer, but it is more viscous. Therefore, the stiffness of the film is 'out of phase' with dissipation. The proposed interpretation seems appealing because it is able to accommodate previous contradictory findings [72].

de Beer et al. experimentally showed that the results depend on the drive frequency, even if the motion of the cantilever base is taken into account in the force inversion procedure [19] (see Fig. 2.16). Their results show that in near-resonance AFM, the measured dissipation is coupled by conservative forces, which results in prominent artificial oscillations in damping. Offresonance AFM, the results are less sensitive to the coupling. In this case, the confined film is bulk-like down to 3 molecular layers and the local maxima in dissipation are out of phase with the maxima interaction stiffness.

In another laboratory, a similar experiment was conducted by FM-AFM with magnetic actuation, which is claimed to be artifact-free [23]. In contrast, the dissipation is shown to be monotonic in the thickness dependence [23]. However, using near-resonance AM with the same driving method, de Beer et al. again observed oscillatory dissipation, which is 'in phase' with the modulation in stiffness [30].

\section{Viscous dissipation in $1 \sim 4$ layers of water}

The debates in the SFA and the AFM community on the dissipation are not restricted to simple liquids like OMCTS, but also include more complicated 
(a)

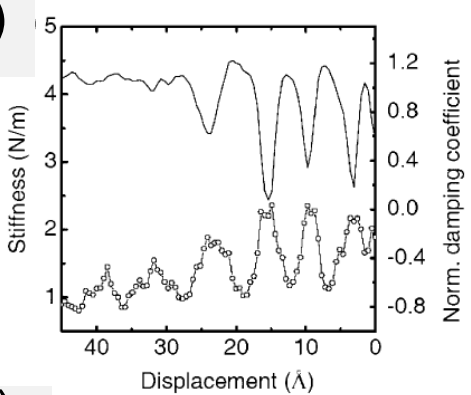

(b)

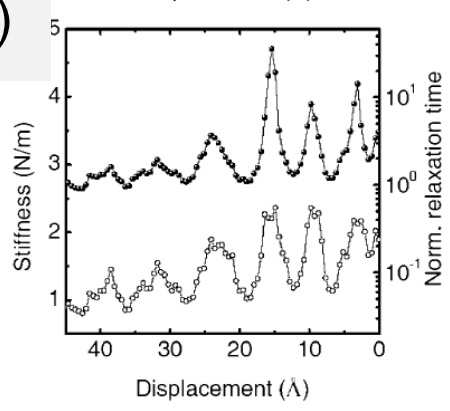

(c)

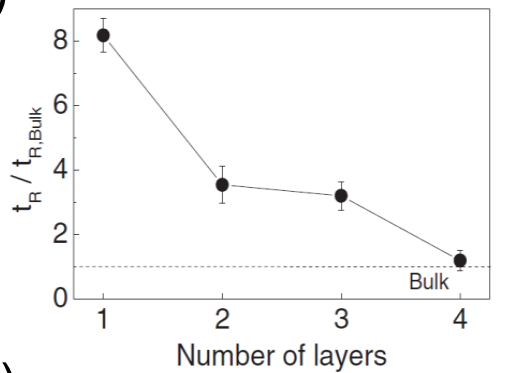

(d)

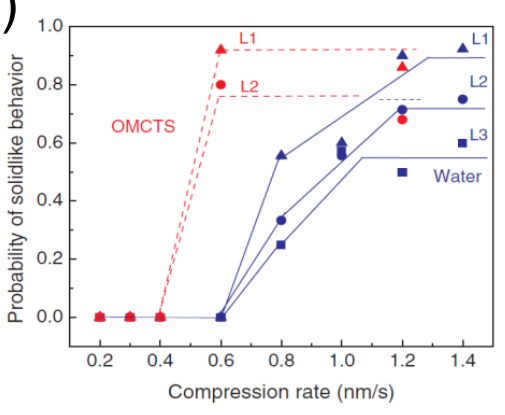

Figure 2.15: (a) The stiffness (open circles) and damping coefficient (line graph) as a function of the OMCTS film thickness. (b) The stiffness (bottom, open circles) and the relaxation time (top, filled circles) calculated through Eq. (2.37) as a function of the OMCTS film thickness. Approach rate $=1.2 \mathrm{~nm} / \mathrm{s}$. (c) The relaxation time of water layers, compared to the bulk. Approach rate $=0.8 \mathrm{~nm} / \mathrm{s}$. (d) Probability of solidification as a function of approach rate (red for OMCTS, blue for Water). (adapted from $[68,69]$ ) 


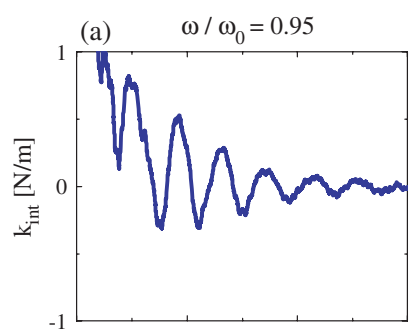

(b) $\quad \omega / \omega_{0}=0.66$

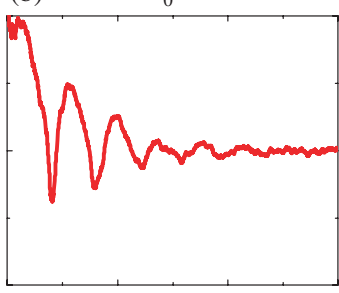

(c)
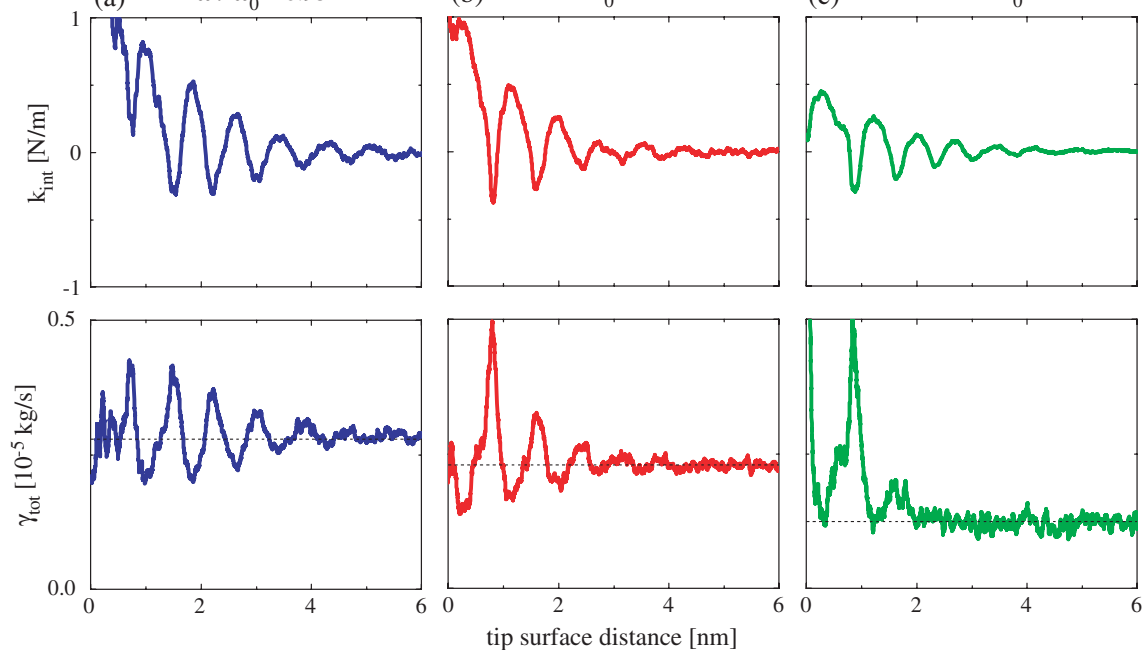

Figure 2.16: The interaction stiffness and damping measured with one cantilever driven at different frequencies. The results with different drive frequencies strongly deviate from each other. (adapted from [19])

liquids like water.

Klein et al. measured the viscosity of water under confinement, using SFA $[73,74]$. It is found that the viscosity of water of the subnanometer thickness is only slightly increased, at most a factor of three. They argued that water under confinement is not in the crystalline state (i.e. ice) because the density of ice is less than water and hence the structure of ice is not favored under confinement. Granick et al. found that friction in confined interfacial water between two crystalline mica surfaces is not isotropic and the shear viscosity oscillates by orders of magnitudes [75]. The argument is that the interfacial water structure is templated (not ice-like though) by the crystalline mica surface despite of the fact that the size of a water molecule does not match with the surface lattice dimension. Using off-resonance AM, Hoffmann et al. found that hydration layers behave like OMCTS under compression [69]. At high compression rates $\left(>0.6 \mathrm{~nm} / \mathrm{s}, R_{t i p} \approx 100 \mathrm{~nm}\right)$, the relaxation time is higher than that of a solid (see Fig. 2.15). And the damping variation with film thickness is also oscillatory as a function of the film thickness. 
(a)

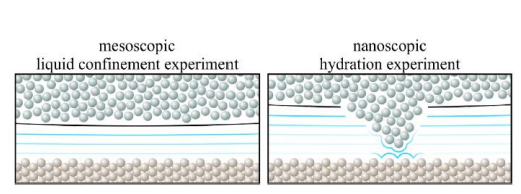

(b)

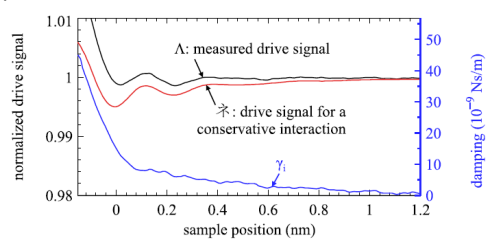

Figure 2.17: (a) The differences between the mesoscopic measurement and the nanoscopic measurement. In the nanoscopic measurement, only a few molecules around the tip apex are probed. (b) The damping and the drive signal as a function of separation. The measurement is carried out in water on mica with a silica tip. (adapted from [25])

In contrast, using FM, Labuda et al. observed monotonic damping with a sharp tip capable of rendering atomic-resolution images [25]. It is argued that the difference in the tip size gives rise to different squeeze-out flows. Under a tip with a few atoms at the apex, the water molecules are squeezedout without collective motion in each layer, which occurs in the case of a mesoscale tip (see Fig. 2.17).

In the same line of reasoning, it is not surprising that SFA and AFM measurements lead to different conclusions due to different confinement geometries. In SFA measurements, liquid is confined between two large atomically flat cylindrical surfaces $(R \approx 1 \mathrm{~cm})$. The contact zone is approximately flat, $>20 \times 20 \mu m^{2}$ (see Fig. 2.14). Nevertheless, in AFM, a tip of nanoscale $(R=1 \sim 100 \mathrm{~nm})$ is used and it may have an atomic scale apex [76], especially for coated ones [30]. The influence of tip shape has been corroborated by Molecular Dynamics (MD) simulations [77].

\subsubsection{Viscous dissipation in overlapping EDLs}

In the previous sections, we introduced electrostatics (EDL) and hydrodynamics (viscous dissipation) individually. Here, we turn our attention to the coupling between electrostatics and hydrodynamics. The coupling was first recognized in the colloid science community [78] and it remains unresolved [79]. Recently, it has drawn interest in the context of micro-/nanofluidics [80-87]. For optimal design of the nanofluidic devices, a deeper understanding of the viscous dissipation at charged interfaces is required, in particular in case of overlapping EDLs originating from two facing surfaces. 
When two charged surfaces are positioned close to each other, screening charges are present in the gap, to neutralize the charges on the surfaces. The EDLs overlap. When a tangential pressure gradient is applied, a streaming current (and potential) will be produced, because the flow carries along a plug of charges [88]. The induced potential drives the charges backwards. The counter flow of charges drags the solvent with them, which is referred to as electroosmosis flow (see Fig. 2.18). As a result, the effective flow rate goes down and thereby the apparent viscosity of the solution increases. The charge-induced enhancement in apparent viscosity is called the electro-viscous effect [89].

Recently, some attempts have been made to harvest energy through the pressure-driven-flow induced streaming potential [81-83, 90-94]. The reported energy conversion efficiency is still not satisfying (usually much less than $10 \%[82,93])$. In the experiments, a hydrostatic pressure gradient is applied across charged nanochannels filled with electrolytes. The classical Helmholtz-Smoluchowski theory is often applied for describing the eletrokinetic phenomena. In the thin double layer limit, the streaming potential is $[95]$

$$
\psi_{s t r}=\frac{\epsilon_{0} \epsilon \zeta \Delta p}{\eta K^{L}}
$$

where $\epsilon \epsilon_{0}$ is permittivity of the bulk, $\Delta p$ is the pressure drop, $\eta$ is the bulk viscosity, and $K^{L}$ is the bulk conductivity, $\zeta$ is the zeta potential of the surface [96]. In the Helmholtz-Smoluchowski theory using a continuum model, many assumptions are made, such as no slip boundary condition, no surface conductance, and no hydration. It has been suggested that surface conductance needs to be incorporated in the interpretation of many electrokinetic phenomena [97]. However, surface conductance is usually not accessible. In conventional techniques (such as streaming potential), the dissipation and the surface potential are often entangled, as shown in Eq. (2.38). AFM force spectroscopy may be a good candidate for exploring the electro-hydrodynamic effect, because it enable us to evaluate conservative forces and viscous dissipation independently. In Chapter 5, we show measurements of viscous dissipation in an EDL and we compare the measured dissipation enhancement with theoretical insights. 


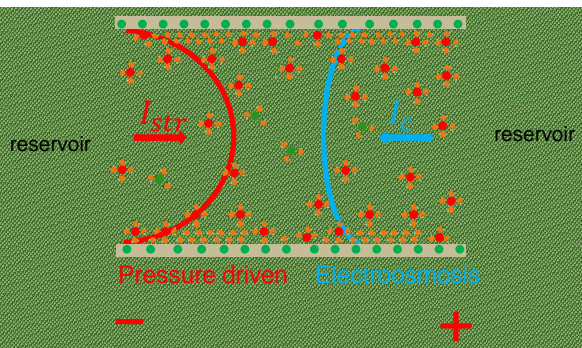

Figure 2.18: Electrokinetic phenomena in the overlapping EDLs. The pressure driven flow produces the streaming current $I_{s t r}$ and the streaming potential. The streaming potential generates a conduction current $I_{c}$. The ions in the conduction current drag along the solvent with them, i.e. the electroosmosis flow.

\subsection{Summary}

In this chapter, we have introduced the principle of AFM and AFM force spectroscopy. We also described thin film mediated forces, including the DLVO forces and viscous dissipation. A brief review on experimental results in thin liquid films shows that the dynamic properties of the thin liquid film are not resolved yet. We reviewed the technical issues met in AFM force spectroscopy in liquid and put emphasis on the necessity of validating available techniques in liquid, in order to establish a reliable technique for application in liquid. Using established techniques as a benchmark, it is possible to examine other techniques and investigate other phenomena, such as electro-hydrodynamic dissipation. 



\section{Bibliography}

[1] Binnig G, Quate C F, and Gerber Ch 1986 Phys. Rev. Lett. 56930

[2] Lang H P, Quate C F, and Gerber Ch 2006 Nature Nanotechnology 13

[3] Gross L, Mohn F, Moll N, Liljeroth P, and Meyer G 2009 Science $\mathbf{3 2 5}$ 1110

[4] Fukuma T, Kobayashi K, Matsushige K, and Yamada H 2005 Appl. Phys. Lett. 87034101

[5] Siretanu I, Ebeling D, Andersson M P, Stipp S L S, Philipse A,Cohen Stuart M, van den Ende D and Mugele F 2014 Sci. Rep. 44956

[6] Butt H J and Jaschke M 1995 Nanotechnology 61

[7] Garcia R and Perez R 2002 Surf. Sci. Rep. 47197

[8] Sader J E 1995 J. Appl. Phys. 8464

[9] Han W, Lindsay S M, and Jing T Appl. Phys. Lett. 694111

[10] Revenko I and Proksch R 2000 J. Appl. Phys. 87526

[11] Ratcliff G C, Erie D A, and Superfine R 1998 Appl. Phys. Lett. 721911

[12] Ramos D, Tamayo J, Mertens J, Calleja M 2006 J. Appl. Phys. 99 124904

[13] Xu X and Raman A 2007 J. Appl. Phys. 102034303

[14] Albrecht T R, Grütter P, Horne D, and Rugar D 1991 J. Appl. Phys. 69668

[15] Giessibl F J 2003 Rev. Mod. Phys. 75949

[16] Sader J E and Jarvis S P 2004 Appl. Phys. Lett. 841801

[17] Sader J E and Jarvis S P 2006 Phys. Rev. B 74195424

[18] de Beer S, van den Ende D, and Mugele F 2008 Appl. Phys. Lett. 93 253106

[19] de Beer S, van den Ende D, and Mugele F 2010 Nanotechnology 21 325703

[20] Kiracofe D and Raman A 2011 Nanotechnology 22485502 
[21] O'Shea S J 2006 Phys. Rev. Lett. 97179601

[22] Schäffer T E, Cleveland J P, Ohnesorge F, Walters D A, and Hansma P K 1996J. Appl. Phys. 803622

[23] Kaggwa G B, Kilpatrick J I, Sader J E, and Jarvis S P 2008 Appl. Phys. Lett. 93011909

[24] Labuda A, Kobayashi K, Kiracofe D, Suzuki K, Grütter P H and Yamada H 2011 Aip Advances 1022136

[25] Labuda A, Kobayashi K, Suzuki K, Yamada H and Grütter P 2013 Phys.Rev. Lett. 110066102

[26] Asakawa H and Fukuma T 2009 Rev. Sci. Instrum. 80103703

[27] Fukuma T, Yoshioka S, and Asakawa H 2011 Rev. Sci. Instrum. 82 073707

[28] Costa L and Rodrigues M S 2015 Beilstein J. Nanotechnol. 6420

[29] Labuda A, Cleveland J, Geisse N A,Kocun M, Ohler B, Proksch R, Viani M B, and Walters D 2014 Microscopy and Analysis 2821

[30] de Beer S, van den Ende D, and Mugele F 2011 J. Phys.: Condens. Matter 2311206

[31] Ebeling D, van den Ende D and Mugele F 2011 Nanotechnology 22 305706

[32] Zhao C, Ebeling D, Siretanu I, van den Ende D, and Mugele F 2015 Nanoscale Doi: 10.1039/C5NR05261K

[33] Iler R K 1979 The Chemistry of Silica (John Wiley and sons, New York)

[34] Lyklema J 1995 Fundamentals of Interface and Colloid Science 1st ed ( Academic press, London)

[35] Ninham B W and Parsegian V A 1971 J. Theor. Biol. 31405

[36] Chan D Y C and Horn R G 1985 J. Chem. Phys. 835311

[37] Carnie S L and Chan D Y C 1993 J. Colloid Interface Sci. 161260

[38] Israelachvili J 1991 Intermolecular and Surface Forces 2nd ed (Academic press, London)

[39] Horn R G and Israelachvili J N 1981 Chem. Phys. Lett. 751400

[40] Abraham F F 1978 J. Chem. Phys. 683713

[41] O'Shea S J, Welland M E, and Rayment T 1992 Appl. Phys. Lett. 60 2356

[42] Parsegian V A and Zemb T 2011 Curr. Opin. Colloid Interface Sci. 16 618

[43] Pashley R M 1981 J. Colloid Interface Sci. 83531

[44] Pashley R M and Israelachvili J N 1984 J. Colloid Interface Sci. 101 511 
[45] Chapel J P 1994 Langmuir 104237

[46] Kilpatrick J I, Loh S H and Jarvis S P 2013 J. Am. Chem. Soc. 135 2628

[47] Williams J A 2005 Engineering Tribology (Cambridge University Press, New York).

[48] Khan S H, Kramkowski E L, Ochs P J, Wilson D M, Hoffmann P M 2014 Appl. Phys. Lett. 104023110

[49] de Beer S, den Otter W K, van den Ende D, Briels W J and Mugele F 2012 Euro Phys. Lett. 9746001

[50] Klein J and Kumacheva E 1995 Science 269816

[51] Klein J and Kumacheva E 1998 J. Chem. Phys. 1086996

[52] Yoshizawa H and Israelachvili J 1993 J. Phys.Chem. 9711300

[53] Thompson P A and Robbins M O 1990 Science 250792

[54] Kohonen M M, Meldrum F C, and Christenson H K 2003 Langmuir 19 975

[55] Ohnishi S, Hato M, Tamada K, and Christenson H K 1999 Langmuir 15 3312

[56] Zhu Y X and Granick S 2003 Langmuir 198148

[57] Israelachvili J, Maeda N, and Akbulut M 2006 Langmuir 222397

[58] Becker T and Mugele F 2003 Phys. Rev. Lett. 91166104

[59] Bureau L 2010 Phys. Rev. Lett. 104218302

[60] Demirel A L and Granick S 1996 Phys. Rev. Lett. 772261

[61] Rosenhek-Goldian I, Kampf N, Yeredor A, and Klein J 2015 P. Natl. Acad. Sci. USA 1127117

[62] Israelachvili J N and Drummond C 2015 P. Natl. Acad. Sci. USA 112 E4973

[63] Dhinojwala A, Bae S C, and Granick S 2000 Tribol. Lett. 955

[64] Demirel A L and Granick S 2002 J. Chem. Phys. 1177745

[65] Jee A Y, Lou K, and Granick S 2015 P. Natl. Acad. Sci. USA 112 E4972

[66] Rosenhek-Goldian I, Kampf N, Yeredor A, and Klein J 2015 P. Natl. Acad. Sci. USA 112 E4974

[67] Maali A, Cohen-Bouhacina T, Couturier G, and Aimé J-P 2006 Phys. Rev. Lett 96086105

[68] Patil S, Matei G, Oral A, and Hoffmann P M 2006 Langmuir 226485

[69] Khan S H, Matei G, Patil S, and Hoffmann P M 2010 Phys. Rev. Lett. 105106101

[70] Hölscher H, Gotsmann B, and Schirmeisen A 2003 Phys. Rev. B 68 153401 
[71] Herruzo E T,Asakawa H, Fukuma T, and Garcia R 2013 Nanoscale 5 2678

[72] Granick S, Bae S C, Kumar S, and Yu C 2010 Physics 373

[73] Raviv U, Laurat P, and Klein J 2001 Nature 41351

[74] Klein J, Raviv U, Perkin S, Kampf N, Chai L, and Giasson S $2004 \mathrm{~J}$. Phys.: Condens. Matter 16 S5437

[75] Zhu Y X and Granick S 2001 Phys. Rev. Lett 87096104

[76] Hiasa T, Kimura K, and Onishi H 2012 Jpn. J. Appl. Phys. 51025703

[77] de Beer S, den Otter W K, van den Ende D, Briels W J and Mugele F 2012 Tribol. Lett. 481

[78] Booth F 1950 Proc. Roy. Soc. A 203533

[79] Rubio-Hernándeza F J, Carriqueb F, and Ruiz-Reinaa E 2004 Adv. Colloid Interface Sci. 51107

[80] Li D Q 2001 Colloid Surf A 19135

[81] van der Heyden F H J, Bonthuis D J, Stein D, and Meyer C, Dekker C 2006 Nano Lett. 62232

[82] van der Heyden F H J, Bonthuis D J, Stein D, Meyer C, and Dekker C 2007 Nano Lett. 71022

[83] Xie Y B, Sherwood J D, Shui L L, van den Berg A, and Eijkel J C T 2011 Lab Chip 114006

[84] Tas N R, Haneveld J, Jansen H V, Elwenspoek M, and van den Berg A 2004 Appl. Phys. Lett. 853274

[85] Haneveld J, Tas N R, Brunets N, Jansen H V, and Elwenspoek M 2008 Appl. Phys. Lett. 104014309

[86] Mortensen N A and Kristensen A 2008 Appl. Phys. Lett. 92063110

[87] Siria A, Poncharal P, Biance A L, Fulcrand R, Blase X, Purcell S T, and Bocquet L 2013 Nature 455494

[88] Hunter R and Leyendekkers J 1978 J. Chem. Soc., Faraday Trans. 174 450

[89] Levine S, Marriott J R, and Robinson K 1975 J. Chem. Soc., Faraday Trans. 271

[90] Yang J, Lu F, Kostiuk L W, and Kwok D Y 2003 J. Micromech. Microeng. 13963

[91] Daiguji H, Yang P D, Szeri A J, and Majumdar A 2004 Nano Lett 4 2315

[92] Sparreboom W, van den Berg A, and Eijkel J C T 2009 Nature Nanotechnology 4713

[93] Wang M R and Kang Q J 2010 Microfluid. Nanofluid. 9181 
[94] Chanda S, Sinha S, and Das S 2014 Soft Matter 107558

[95] Lyklema J 2014 Colloids and Surfaces A: Physicochem. Eng. Aspects 440161

[96] Hunter R J, Ottewill R H, and Rowell R L 1981 Zeta Potential in Colloid Science (Academic press, London)

[97] Lyklema J 2001 J. Phys.: Condens. Matter 135027 



\section{Chapter 3}

\section{Atomic force microscopy of confined liquids using the thermal bending fluctuations of the cantilever}

We use Atomic Force Microscopy (AFM) to measure the distance-dependent solvation forces and the dissipation across liquid films of octamethylcyclotetrasiloxane (OMCTS) confined between a silicon tip and a highly oriented pyrolytic graphite (HOPG) substrate without active excitation of the cantilever. By analyzing the thermal bending fluctuations, we minimize possible non-linearities of the tip-substrate interaction due to finite excitation amplitudes because these fluctuations are smaller than typical one Angstrom, much smaller than the characteristic interaction length. Moreover, we avoid the need to determine the phase lag between cantilever excitation and response, which suffers from complications due to hydrodynamic coupling between cantilever and fluid. Consistent results, and specially high quality dissipation data, are obtained by analyzing the power spectrum and the time autocorrelation of the force fluctuations. We validate our approach by determining the bulk viscosity of OMCTS using tips with a radius of approximately $1 \mu \mathrm{m}$ at tip-substrate separations $>5 \mathrm{~nm}$. For sharp tips we consistently find an ex- 
Chapter 3. Atomic force microscopy of confined liquids using the thermal

ponentially decaying oscillatory tip-substrate interaction stiffness as well as a clearly non-monotonic variation of the dissipation for tip-substrate distances up to 8 and $6 \mathrm{~nm}$, respectively. Both observations are in line with the results of recent simulations which relate them to distance dependent transitions of the molecular structure in the liquid.

\subsection{Introduction}

Understanding the properties of nano-confined liquids is of great importance in numerous research fields, like biophysics [1] and nanofluidics [2], and industrial applications, such as friction, wear and lubrication $[3,4]$. In particular, what happens when we squeeze-out a liquid between atomically flat surfaces? At distances significantly larger than the molecule size, the drainage process is described by the well-known Reynolds approximation [5] of the NavierStokes equations. However, for nano-confined liquids, where the film thickness is comparable to the molecular size, continuum physics breaks down and the liquid film is squeezed out layer by layer $[6,7]$. These discrete transitions and the layering configuration are caused by the molecular self-assembly of the liquid close to solid walls. Upon confinement this gives rise to the conservative oscillatory solvation forces [8]. These solvation forces were first measured in the 1980s [9] and are by now well-established [10-27]. However, how molecular self-assembly affects the dynamics of the confined liquid is still heavily debated due to contradicting experimental results.

In Surface Forces Apparatus (SFA) experiments, confinement-induced solidification was observed when shearing the confined liquid [28,29]. Other studies [13] reported a viscoelastic shear response akin to jamming. Measurements of the rupture process of squeezing out the liquid layer by layer could be described using a discretized version of the Navier-Stokes equations with a more or less bulk-like viscosity down to the last two layers $[14,20]$. Recent experiments [22] and theoretical studies [30] indicate that some of these apparent inconsistencies can be traced down to the strong structural anisotropy in the confined liquid, which can lead to a highly anisotropic effective viscosity.

In more recent Atomic Force Microscopy (AFM) experiments with confined liquids similar discrepancies have been observed. While some studies report a monotonic increase in the viscous dissipation [10,19, 26, 27], others detect distance dependent features in the dissipation $[11,12,17,18,21,23-25$, 31,32]. All these measurements were performed using various forms of dynamic AFM spectroscopy using actively driven cantilevers. Several problems 
may contribute to the discrepancies of the results reported in the literature. In liquid the cantilever is subject to a strong mechanical coupling with the fluid. As a consequence, the cantilever suffers from viscous friction with the ambient fluid leading to a low overall quality factor of order unity. The damping due to the confined liquid is only a rather small addition to the overall damping. Moreover, the coupling between the cantilever, the fluid, and the surrounding liquid cell can give rise to additional resonances leading to the well-known problem of a 'forest of peaks', in particular for acoustically driven cantilevers $[26,33]$.

Optimizations of the cantilever holder $[34,35]$, dynamic models taking into account the base motion of the cantilever [26,36], as well as other (e.g. magnetic) driving schemes [37-39] help to reduce these problems, yet, they do not overcome the fundamental problem that the reconstruction of the force is based on an inversion of the measured amplitude and phase (or resonance frequency) and requires an accurate model of the cantilever dynamics including in particular knowledge and calibration of the phase lag between driving and response $[40,41]$. In addition, the finite drive amplitude (e.g. in excess of the molecular diameter) may 'smear out' variations of interaction and dissipation forces on smaller length scales [24].

To avoid these difficulties, we revisit the analysis of the thermal noise signal to study the conservative and dissipative properties of confined liquids [15,42-47]. This method, which has by now become a standard tool to determine the cantilever spring constant [42], minimizes the external perturbation of the system and it eliminates the need of a phase measurement. We investigate the influence of the tip-substrate interaction on the thermal noise signal to determine both the distance-dependent interaction stiffness due to conservative (oscillatory) tip-substrate interactions $[15,43,44]$ and the interaction damping due to the local energy dissipation near the substrate. Our approach extends earlier studies of visco-elastic properties of polymeric systems [45-47] and shear forces in confined liquids [48]. We record time series of the noise signal using a high speed and broadband data capture and analyse both their power spectral density (PSD) as well as the time autocorrelation function (ACF) [49]. Notwithstanding earlier reports of differences regarding effect of electronic noise [50] we find that both approaches yield consistent results, as expected based on the Wiener-Khinchin theorem [51,52].

The conservative force and dissipation in the confined liquid are determined alternatively by fitting the obtained power spectra and autocorrelation of the fluctuating tip displacement to a simple harmonic oscillator (SHO) model of the cantilever. The amplitude of the cantilever motion, typically 50 pm at room temperature, is significantly smaller than that in dynamic AFM, 
Chapter 3. Atomic force microscopy of confined liquids using the thermal

thereby minimizing sample perturbation and ensuring the applicability of linear response. To validate the thermal noise method we first determine the bulk viscosity of the liquid by measuring the hydrodynamic dissipation using relatively large cantilever tips, with a radius of about $1 \mu \mathrm{m}$. This verification is referred to as Reynolds damping measurements in this chapter. Next, we study the distance dependent interaction forces and dissipation, using smaller tips with a radius of about $50 \mathrm{~nm}$. Both the observed stiffness and damping oscillate as a function of the tip-substrate distance at distances below $6 \mathrm{~nm}$ as a consequence of the layering effects, in agreement with statistical physics [53] and molecular dynamics (MD) simulations [54].

\subsection{Methods}

\subsubsection{Materials}

The experimental AFM set-up to obtain the thermal fluctuations as a function of tip-substrate distance is shown in Fig. 3.1(a). While ramping up and down the substrate close to the tip as slow as possible, we monitor the fluctuations of the deflection signal. As substrate we use freshly cleaved and atomic flat highly oriented pyrolytic graphite, HOPG, with an in plane lattice constant of $0.25 \mathrm{~nm}$ (Mikromasch grade ZYB). For the liquid we choose octamethyl-cyclotetrasiloxane (OMCTS, as received from Fluka, purum $\geq$ $99.0 \%$ ) because its molecules have a relatively large diameter of $0.8 \sim 0.9 \mathrm{~nm}$. The AFM device itself is a Veeco Multimode 8 with a Nanoscope V controller and Veeco EV scanner. The AFM is housed in an acoustic isolation box and operated at a constant temperature of $300 \mathrm{~K}$. For the nano-confinement measurements cantilevers with a sharp tip were used, from Mikromasch, NSC36 aluminum coated on the back side. They have a spring constant of $k_{c}=1 \sim 4$ $\mathrm{N} / \mathrm{m}$, as determined in air using the thermal calibration method [42], and a resonance frequency $f_{r}$ in liquid between 45 and $80 \mathrm{kHz}$, as determined $8 \mathrm{~nm}$ away from the substrate. For the Reynolds damping measurements cantilevers with a relatively blunt silicon tip were used, from Team nanotech, which were LRCH coated with aluminum on the back side. These have a $k_{c}$ of $\sim 2 \mathrm{~N} / \mathrm{m}$ and a $f_{r}$ in liquid of $\sim 25 \mathrm{kHz}$. Prior to the experiments cantilevers and fluid cell are rinsed in isopropanol and ethanol, after which the cantilevers are treated for one minute in a plasma-cleaner (Harrick Plasma). After the measurements the tips are characterized by high resolution SEM (HR-SEM Zeiss LEO 1550) to estimate the tip radius and to make sure that the cantilevers were clean [55]. The sharp tips turned out to have radii of 


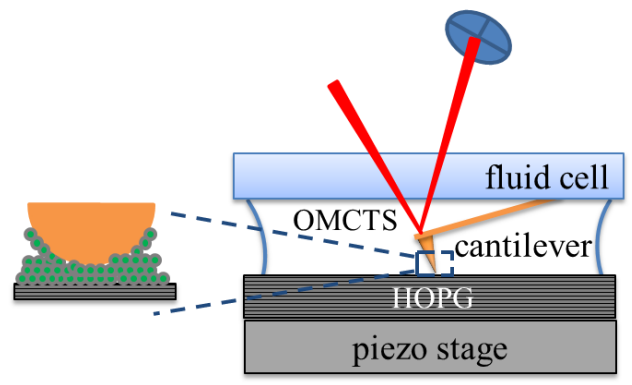

(a)

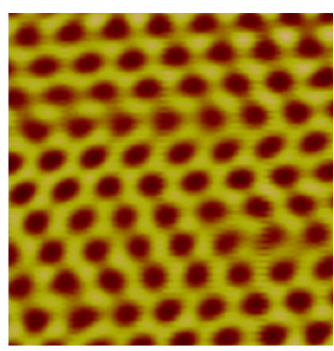

(b)

Figure 3.1: (a) Schematic representation of the experimental set-up.

Friction microscopy image of HOPG $(2 \mathrm{~nm} \times 2 \mathrm{~nm})$ after Fourier filtering.

$30 \sim 70 \mathrm{~nm}$, the blunt tips $\sim 900 \mathrm{~nm}$.

\subsubsection{Experimental procedures}

As stated above, two kinds of experiments are conducted: one is done with blunt tips to validate the thermal noise approach by measuring the Reynolds damping; the other is done with sharp tips to probe the dynamics in the layered liquid. The experimental procedures are identical, but the experimental parameters are slightly different.

The AFM is operated in force-distance mode. While the distance between cantilever tip and substrate is varied periodically at low speed, the deflection signal $z(t)$ is monitored at a sampling rate of $500 \mathrm{kHz}$ (i.e. over 6 times the cantilever's fundamental eigenfrequency) using a low pass filter with a bandwidth of $200 \mathrm{kHz}$ to prevent aliasing. To correct for the drift of the piezo stage, due to thermal expansion or creep, we use a fixed $(2 \sim 4 \mathrm{~nm})$ maximum deflection of the cantilever as set point for the highest position of the stage, i.e. as retraction threshold, after which the stage is retracted backwards over a fixed distance before next approach is started. From the variations in the approach distances we estimate that the drift during the measurements is always less than $160 \mathrm{pm} / \mathrm{s}$. Therefore we choose an approach speed of $1 \mathrm{~nm} / \mathrm{s}$ and a ramp size of $10 \mathrm{~nm}$ for the measurements with the 
Chapter 3. Atomic force microscopy of confined liquids using the thermal 48

bending fluctuations of the cantilever

sharp tips. During measurements with blunt tips, i.e. the Reynolds damping measurements, the retraction speed, distance and threshold are $8 \mathrm{~nm} / \mathrm{s}, 25$ $\mathrm{nm}$, and $4 \mathrm{~nm}$, respectively. In both cases, these parameters guarantee data acquisition time at acceptable drift.

In the case of sharp tips, the retraction threshold of $2 \mathrm{~nm}$ ensures hard contact between the tip and the sample, i.e. all OMCTS will be squeezed out. This is concluded from friction force images recorded at the same deflection set point, which corresponds to a load of $\sim 5 \mathrm{nN}$. As shown in Fig. 3.1(b), these data reveal a hexagonal lattice (lattice constant of $0.25 \mathrm{~nm}$ ) characteristic for HOPG.

\subsubsection{Model}

Although the single harmonic oscillator (SHO) model is not fully appropriate to describe the cantilever dynamics over a wide frequency range, see Section 3.4 and the Appendix for discussion of this issue, the deviation from the full solution is only a few percent within our fitting range, so this approach is more than sufficient. The SHO is driven by the Brownian force along with tip-substrate interactions:

$$
m^{*} \ddot{z}+\gamma_{c} \dot{z}+k_{c} z=F_{t s}+F_{B}(t)
$$

where $z$ is the tip position, $m^{*}$ the total effective mass (including the added mass originating from the motion of the surrounding liquid), $\gamma_{c}$ the viscous damping around the cantilever, $k_{c}$ the intrinsic cantilever stiffness, $F_{t s}$ the distance dependent tip-substrate interaction, and $F_{B}$ is the random force due to Brownian motion, characterized by $\left\langle F_{B}\right\rangle=0$ and $<F_{B}(s) F_{B}(s+t)>=2 \gamma k_{B} T \delta(t)$, where $k_{B} T$ is the thermal energy and $\delta(t)$ the Dirac delta function. According to the equipartition theorem, the average potential energy of the cantilever, $\frac{1}{2} k\left\langle z^{2}\right\rangle$, is equal to $\frac{1}{2} k_{B} T$, so for a cantilever stiffness of $2 \mathrm{~N} / \mathrm{m}$ the root-mean-square displacement of the unperturbed thermal motion is around $46 \mathrm{pm}$ at room temperature. This amplitude is much smaller than characteristic length scale of the variations in the tip-substrate interaction, which for OMCTS is about $0.9 \mathrm{~nm}$. Therefore, linearization of the tip-substrate force around the average tip displacement is justified, i.e. $F_{i n t}=F_{0}-k_{i n t} z-\gamma_{\text {int }} \dot{z}$, where $k_{i n t}$ is the interaction stiffness, $\gamma_{\text {int }}$ the interaction damping, and Eq. (3.1) can be rewritten as:

$$
m^{*} \ddot{z}+\gamma \dot{z}+k z=F_{B}(t)
$$

where $z$ is now the tip displacement with respect to its average position at distance $d, \gamma=\gamma_{c}+\gamma_{i n t}$ is the total damping coefficient and $k=k_{c}+k_{\text {int }}$ 
the total stiffness. Solving Eq. (3.2) in the frequency domain, we get:

$$
z[\omega]=\left(k-m^{* 2}+j \gamma \omega\right)^{-1} F_{B}[\omega]
$$

where $j=\sqrt{-1}$ is the imaginary unit. The PSD of the displacement $\mathrm{z}$ is defined as:

$$
P_{z z}(\omega)=\lim _{t_{s} \rightarrow \infty}\left(\frac{1}{t_{s}} z[\omega] z[\omega]^{*}\right)
$$

where $t_{s}$ is the sampling time. According to the Wiener-Khinchin theorem $[51,52]$ the PSD of the Brownian force $F_{B}$ is related to its ACF as $P_{F F}(\omega)=$ $2 \gamma k_{B} T$. Hence, $P_{z z}(\omega)$ can be rewritten as:

$$
P_{z z}(\omega)=P_{w}+\frac{P_{o}}{\left(1-\left(\frac{\omega}{\omega_{r}}\right)^{2}\right)^{2}+\left(\frac{\omega}{\omega_{r} Q}\right)^{2}}
$$

where $P_{w}$ accounts for the instrumental (white) noise; $\omega_{r}$ is the resonance frequency, $Q$ the quality factor and $P_{o}$ a scaling factor. Both $Q$ and $\omega_{r}$ depend on tip-substrate distance and are related to $k_{\text {int }}$ and $\gamma_{\text {int }}$ by:

$$
\begin{aligned}
& k=k_{i n t}+k_{c}=m^{*} \omega_{r}{ }^{2}, \\
& \gamma=\gamma_{i n t}+\gamma_{c}=m^{*} \omega_{r} / Q .
\end{aligned}
$$

The effective mass $m^{*}$ is estimated by substituting the intrinsic cantilever stiffness $k_{c}$ and the resonance frequency $\omega_{r}$, as measured in liquid away from the substrate, where the tip-substrate interaction can be neglected, into Eq. (3.2). As stated before the stiffness $k_{c}$ itself is obtained from the thermal calibration procedure in air.

Another way to determine the interaction parameters is to measure the ACF of the displacement signal $z(t)$, defined as:

$$
R_{z z}(t)=\frac{1}{t_{s}} \int_{0}^{t_{s}} z(s) z(s+t) d s .
$$

Since $R_{z z}(t)$ is equal to the inverse Fourier transform of the PSD, for $Q>0.5$ one can show that $[56]$ :

$$
R_{z z}(t)=\frac{k_{B} T}{m^{*} \omega_{r}{ }^{2}} \exp \left(\frac{-\omega_{r} t}{2 Q}\right)\left(\cos \omega_{1} t+\frac{\sin \omega_{1} t}{\sqrt{(2 Q)^{2}-1}}\right)+R_{0}
$$

where $\omega_{r}=\sqrt{k / m^{*}}, \omega_{1}=\omega_{r} \sqrt{\left(1-(2 Q)^{-2}\right)}$, and $R_{0}$ a constant to compensate for a possible background. By fitting Eq. (3.7) to the measured ACF one again obtains values for $Q$ and $\omega_{r}$. 
Chapter 3. Atomic force microscopy of confined liquids using the thermal

\subsection{Data analysis}

After we have recorded the tip displacement as a function of time during ramping, this time sequence $z\left(t_{n}\right)$ is split up into small time intervals, each of which contains $2^{16}$ data points. From the displacement versus time sequence in each interval the background, i.e. best fitting straight line, is subtracted before the time sequence is transformed into a power spectral density, Eq. (3.3), using a standard fast-Fourier-transform algorithm to calculate $z[\omega]$. Next, our model, Eq. (3.4) is fitted to the obtained $P_{z z}(\omega)$, revealing values for $k_{\text {int }}(d)$ and $\gamma_{\text {int }}(d)$ where $d$ is the average tip-substrate distance during the considered time interval. Alternatively, the ACF of the time sequence is calculated with Eq. (3.6) and our model function, Eq. (3.7), is fitted to this correlation, again resulting in best fit values for $k_{i n t}(d)$ and $\gamma_{i n t}(d)$.

Although this analysis is straightforward, one has to keep in mind two aspects. Firstly, the accuracy of the obtained values for $Q$ and $\omega_{r}$ depends strongly on $Q$ and the number of data points $N$ in the time sequence. For $N=$ $2^{16}$ and $Q>1.5$ we obtain $\Delta Q / Q<0.1$ and $\Delta \omega_{r} / \omega_{r}<0.01$ during fitting. The accuracy is further improved by averaging over several, typically 50 , approach curves. For $Q<1$ the system is overdamped and the uncertainty in both $Q$ and $\omega_{r}$ strongly increases with decreasing $Q$ value. In the case of sharp tips in our experiments, this occurs at $d<1.5 \mathrm{~nm}$. Hence, the method gives reliable results for distances larger than $1.5 \mathrm{~nm}$. Secondly, during the sampling of a time sequence the substrate is not stationary but travels $0.13 \mathrm{~nm}$; the tip-substrate distance changes between $0.07 \mathrm{~nm}$ and $0.24 \mathrm{~nm}$ depending on the tip-substrate interaction at that distance. In all cases, this variation is sufficiently small compared to the diameter of the OMCTS molecules, i.e. the length scale on which the tip-substrate interaction is expected to vary.

\subsection{Results}

We first discuss the validation of our method using the Reynolds damping measurements with blunt tips of radius $R_{t i p} \approx 900 \mathrm{~nm}$. The inset of Fig. 3.2(a) gives three exemplary power spectra with best fit curves at various distances indicated by the (colour matched) arrows in the main panel. The spectra are extracted from 25 approach curves, measured consecutively with the same tip. As the distance decreases, the peaks in the power spectra become broader, i.e. the fitted $Q$ values become smaller, and the resonance shifts towards lower frequencies. From the fitted $Q$ values we calculate the 


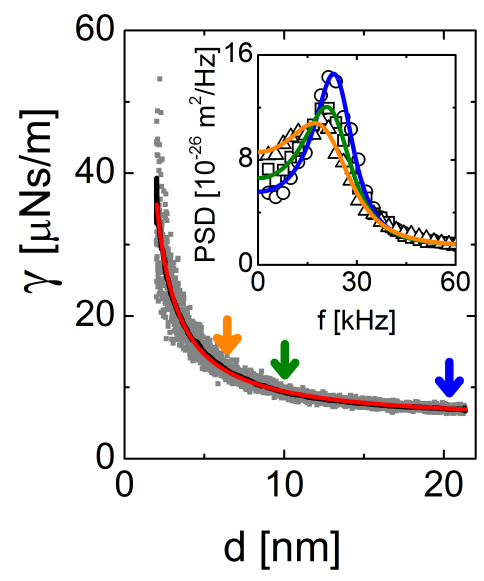

(a)

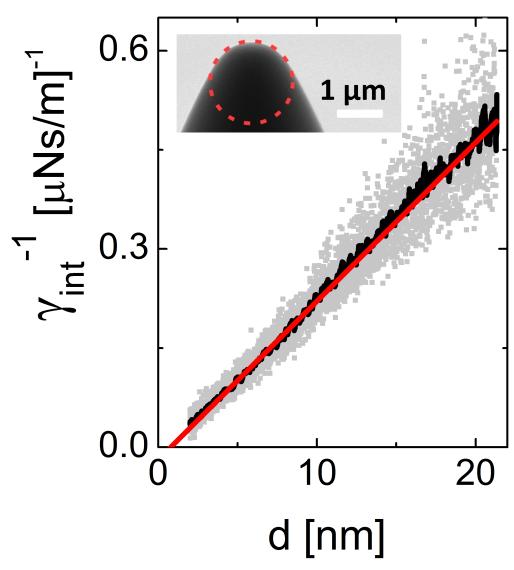

(b)

Figure 3.2: (a) Damping coefficient versus tip-substrate distance extracted from 25 approaches. Black line: averaged data, using a 25 points moving average. Red curve: best fit (Eq. 3.8) to the data for damping coefficient. The inset shows the measured (smoothed) PSD (symbols) at three different distances - denoted by the arrows in the main graph (solid lines are fits to our model function). (b) Inverse of Reynolds damping coefficient as a function of tip-substrate distance. Black line: averaged data, using a 25 points moving average. Red line: linear fit curve. The inset shows the SEM image of the tip used in the experiments $(R=900 \mathrm{~nm}$, red circle). Parameter values of cantilever: $k_{c}=1.91 \pm 0.06 \mathrm{~N} / \mathrm{m} ; f_{r}=\omega_{r} / 2 \pi=25.2 \pm 0.3 \mathrm{kHz}$ in liquid at $d \approx 21 \mathrm{~nm}\left(m^{*}=7.61 \times 10^{-11} \mathrm{~kg}\right)$.

damping coefficient using Eq. (3.5b). The total damping increases monotonically with decreasing tip-substrate distance. The expected hydrodynamic Reynolds damping due to the confined liquid under the tip is given by

$$
\gamma(d)=\gamma_{c}+6 \pi \eta \frac{R_{t i p}^{2}}{d-\Delta}
$$

where $\gamma_{c}$ is the damping experienced by the cantilever beam, and $\Delta$ is an offset to compensate for the error in zero separation. $\eta$ is the viscosity of the liquid.

To ensure bulk behavior we exclude data at distances less than $5 \mathrm{~nm}$ 
Chapter 3. Atomic force microscopy of confined liquids using the thermal

from the fit. This lower limit is obtained from the results of measurements with a sharp tip, see below. Fitting Eq. 3.8 to our data, we find a viscosity $\eta=2.7 \pm 0.2 \mathrm{mPa} \cdot \mathrm{s}$, in agreement with literature data: $2.2 \sim 2.5 \mathrm{mPa} \cdot \mathrm{s}$ $[24,57]$. For $\Delta$ we obtain a value of about $0.8 \mathrm{~nm}$, which indicates that an equivalent of one molecular layer remains rigidly stuck to one of the two the solid surfaces in qualitative agreement with earlier SFA measurements $[6,14]$. To examine the quality of the fit, the inverse of the Reynolds damping has been plotted versus tip-substrate distance in Fig. 3.2(b). The inverse damping indeed shows a linear behaviour, down to the smallest tip-substrate distances [58]. From this observation we conclude that the choice of the lower bound of $5 \mathrm{~nm}$ in our analysis is not critical and that slip is absent in our system (as expected for a complete wetting system).

We also validated the conservative tip-substrate forces by extracting the force gradient from the distance-dependent resonance frequency. For separations larger than $9 \mathrm{~nm}$, we find a monotonically increasing attraction that can be described by van der Waals interaction between the tip and graphite substrate across the liquid film (see Fig. S1 in Supplementary Information 3.A.2). At $d<6 \mathrm{~nm}$, the attraction becomes repulsive, with a weak oscillatory component superimposed. While the oscillation has the expected periodicity corresponding to molecular diameter, the features are not very pronounced, presumably due to the poorly defined geometry of the large tips on small scales.

Overall, these validation measurements demonstrate that our method does yield quantitatively correct values for the forces, including in particular dissipative forces down to a few nanometers tip-substrate separation. They provide a crucial link between well-established continuum physics and the nano-scale behaviour to be described below, which has proven difficult to achieve in many earlier studies of confined liquids.

In Figs. 3.3-3.5 we present the tip-substrate interactions measured with a sharp tip close to the substrate. The main panel of Fig. 3.3 shows the average deflection of the cantilever during approach up to the retraction threshold of 2 $\mathrm{nm}$. The insets show the measured PSDs (right) and ACFs (left) at three different distances indicated by the (colour matched) arrows in the main graph. The solid lines represent our model fits (Eqs. (3.4) and (3.7)) from which we extract the values of $\omega_{r}$ and $Q$. At distances corresponding to positive (negative) force gradients, as denoted by an olive (blue) arrow, the resonance frequency is shifted towards larger (smaller) frequencies. Correspondingly, the magnitude of the autocorrelation function is reduced (increased) and the position of the first zero crossing is shifted towards shorter (larger) times, in 


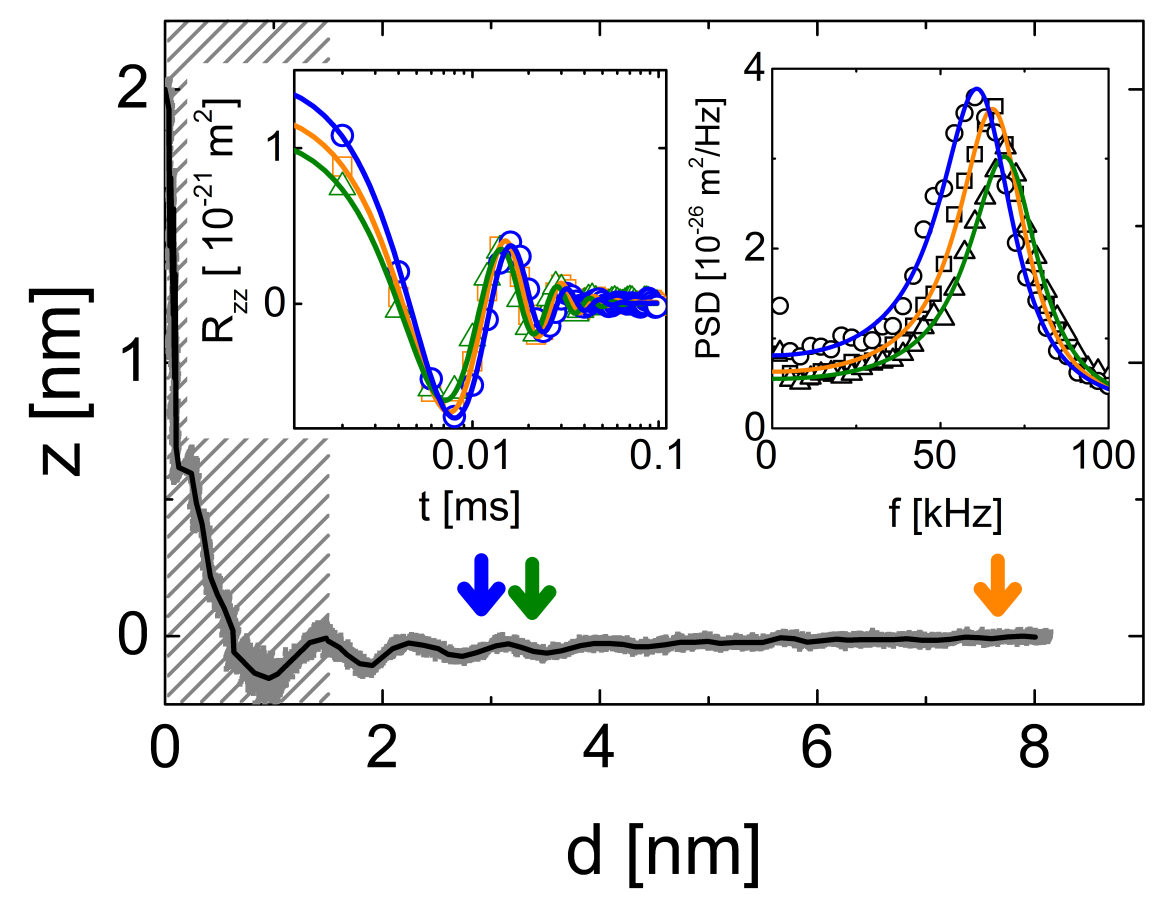

Figure 3.3: Measured cantilever deflection (grey) upon approach of the tip towards the surface at $1 \mathrm{~nm} / \mathrm{s}$ (black line, averaged data, using a $2^{16}$ point moving average). The inset shows the measured autocorrelations $R_{z z}$ and measured (smoothed) power spectrum density PSDs (symbols) at three different distances denoted by the arrows in the main graph (solid lines are fits to our model function). Data at $d<1.5 \mathrm{~nm}$ are shadowed, because they are not taken into account in further analysis, see Figs. 3.4 and 3.5. Cantilever parameters: $R_{t i p}=45 \mathrm{~nm}, k_{c}=2.71 \pm 0.08 \mathrm{~N} / \mathrm{m} ; f_{r}=67.5 \pm 0.2 \mathrm{kHz}$ and $Q=2.82 \pm 0.08$ at $d=8 \mathrm{~nm}\left(m^{*}=1.51 \times 10^{-11} \mathrm{~kg}\right)$. 
Chapter 3. Atomic force microscopy of confined liquids using the thermal

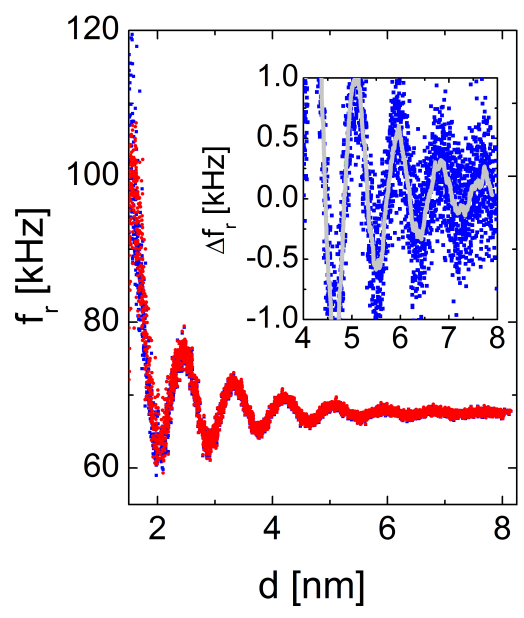

(a)

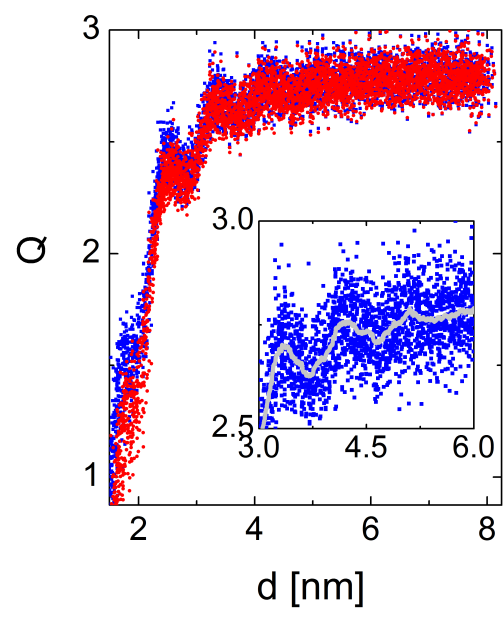

(b)

Figure 3.4: Fitted resonance frequency (a) and quality factor (b) versus tipsubstrate distance extracted from 97 approaches, blue squares by the PSD analysis, red dots by the ACF analysis. (a) The inset shows the resonance frequency shifts and also the averaged one extracted by the PSD analysis. (b) The inset shows the fitted quality factor and the averaged one by PSD analysis.

agreement with our expectations. For values of $d<1.5 \mathrm{~nm}$, the cantilever motion becomes overdamped and the fits to both the PSD and ACF no longer yield reliable values.

We note here that the PSDs shown in Fig. 3.2(a) and Fig. 3.3, display a small excess power at low frequencies, compared to the SHO fit. At the same time, we slightly shift the ACFs vertically to make them decay to zero for long times. We will explain the origin of these effects in the discussion section.

In Fig. 3.4, we plot the values for (the shift in) the resonance frequency $\omega_{r}$ and the quality factor $Q$ obtained from the analysis the PSD and the ACF as a function of $d$. The data represent an average of 97 approach curves. The individual curves were aligned by shifting the two peak positions at $\sim 2.4$ $\mathrm{nm}$ and $\sim 3.3 \mathrm{~nm}$ in the resonance frequency profile (see Fig. 3.4) on top of each other to compensate for residual drift. The offset per curve is typically 


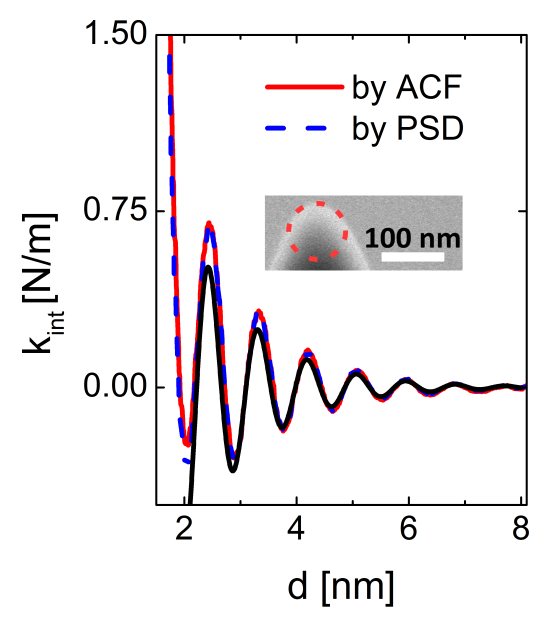

(a)

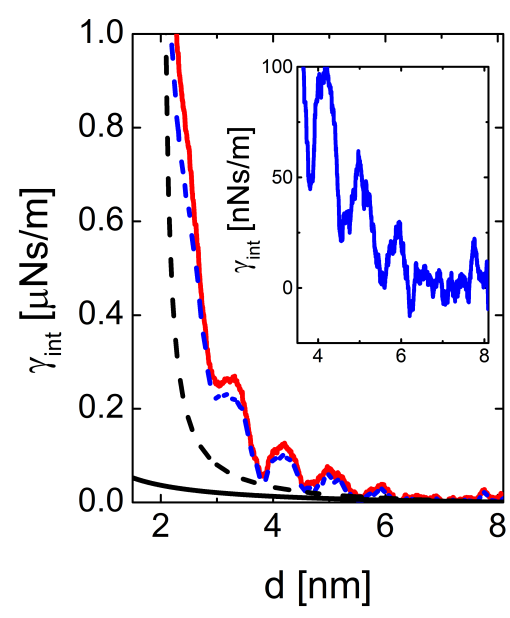

(b)

Figure 3.5: Interaction stiffness (a) and damping (b) versus distance. Blue lines: results from the PSD analysis, red lines: results from the ACF analysis. Both are smoothed using a 97 point moving average. (a) Solid black line: best fit of the oscillatory decaying stiffness profile. The inset shows an SEM image of the tip. (b) Solid black line: calculated Reynolds damping, using the reference bulk viscosity and the tip radius obtained from the SEM image. Dashed black line: obtained by shifting the black solid line $2 \mathrm{~nm}$ to the right. Both calculated lines are offset vertically so that they are aligned to experimental data at $8 \mathrm{~nm}$. The inset shows a zoom-in of the average damping coefficient obtained from the PSD analysis.

smaller than $0.1 \mathrm{~nm}$ (see Fig. S2 of the Supplementary Information 3.A.2). Figure 3.4 clearly shows that the results from ACF and PSD analysis are in perfect agreement. Both methods are thus equally viable and allow for detecting oscillatory solvation forces up to distances of at least $8 \mathrm{~nm}$, as the variation of the resonance frequency shows. The inset of Fig. 3.4(a) suggests that we can detect variations of the resonance frequency down to values of $\Delta f \approx 50 \mathrm{~Hz}$.

In Fig. 3.5, we show the interaction stiffness $k_{\text {int }}(d)$ and the interaction damping $\gamma_{i n t}(d)$ as extracted from data in Fig. 3.4 using Eq. (3.5) (assuming $\gamma_{\text {int }}=0$ at $d=8 \mathrm{~nm}$ ). The shape of the conservative solvation force gradient 
Chapter 3. Atomic force microscopy of confined liquids using the thermal

is empirically described $[6,8]$ :

$$
k_{i n t}(d)=K_{\text {int }} \exp \frac{-(d+\Delta d)}{\epsilon} \cos \left(\frac{2 \pi(d+\Delta d)}{\sigma}\right)
$$

Here $\sigma=0.88 \mathrm{~nm}$ is the periodicity determined directly from the oscillatory profile; the scaling factor $K_{\text {int }}$, the decay length $\epsilon$ and the offset $\Delta d$ are obtained from the best fit. Data at a distance less than $2 \mathrm{~nm}$ are excluded from the procedure, because the uncertainty in $f_{r}$ and $Q$ is too large. The black curve in Fig. 3.5(a) is the best fit profile with $K_{\text {int }}=3.6 \pm 0.1$ $\mathrm{N} / \mathrm{m}, \epsilon=1.39 \pm 0.02 \mathrm{~nm}$ and $\Delta d=0.194 \pm 0.001 \mathrm{~nm}$. To convert the measured interaction stiffness $k_{\text {int }}$ to a normalized interaction force $F / R$ we integrate the local oscillatory pressure $p(x)=-p_{\infty} \cos (2 \pi x / \sigma) \exp (-x / \epsilon)$ using the Derjaguin approximation [10], to obtain $F(d)=2 \pi R \int_{d}^{\infty} p(x) d x$ where $x(r)=d+r^{2} /(2 R)$. From this calculation we obtain an amplitude $F / R=9 \pm 3 \mathrm{mN} / \mathrm{m}$, where the tip radius $R$ is characterized by SEM imaging (see SEM image of the tip after use in Fig. 3.5(a)). The periodicity $\sigma$, the decay length $\epsilon$ and the normalized interaction force $F / R$ are in good agreement with earlier measurements using SFA or AFM $[8,24]$.

The interaction damping features clear oscillations superimposed onto an overall increasing background, as shown in Fig. 3.5(b). Local maxima and minima in the dissipation can be clearly distinguished between $d=2 \mathrm{~nm}$ and $6 \mathrm{~nm}$, i.e. over a much larger range than reported previously [24]. The location of the local maxima coincides with the maxima in the interaction stiffness in Fig. 3.5(a).

The absolute value of the dissipation is approximately 10 times higher than the expectation based on the prediction of Reynolds theory, i.e. continuum hydrodynamics in combination with the bulk viscosity and $\Delta=0$ in Eq. (3.8), which is shown as a solid line in Fig. 3.5(b). If we follow earlier suggestions $[9,14]$ to include a stagnant layer of finite thickness, the thus corrected continuum model comes closer to the experimental data, as exemplified by the dashed line in Fig. 3.5, which corresponds to $\Delta=2 \mathrm{~nm}$. Therefore, it is worth noting that for comparing the experimental data to the continuum model, it is critical to take into account the (non)existence of stagnant layers.

\subsection{Discussion}

Before we discuss our results, also in relation to earlier studies, we firstly consider the advantages and disadvantages of the thermal noise approach in 
more detail.

Approximating the cantilever as an SHO - Although in the SHO approach the cantilever is modeled as a point mass attached to a massless spring it is rather accurate in describing the lowest vibrational mode of a cantilever [59]. Because in the lowest oscillation mode all parts of the cantilever oscillate perfectly in phase with the tip. Hence, effective values for the mass $m^{*}$, damping $\gamma_{c}$ and stiffness $k_{c}$ of the cantilever, which can all be determined by calibration measurements, are sufficient to describe the cantilever dynamics. However, the cantilever bending depends also weakly on the external load on the tip. This causes a small systematic error. Yet, a decomposition of the cantilever into small segments for typical parameter values $(\mathrm{Q} \sim 3)$ shows that the relative error in the interaction parameters $k_{\text {int }}$ and $\gamma_{\text {int }}$ with respect to $k_{c}$ and $\gamma_{c}$, is never larger than typically $3 \%$ for $\left|k_{i n t} / k_{c}\right|<0.9$ and $\left|\gamma_{i n t} / \gamma_{c}\right|<0.8$ (see also Appendix). The experimental error in the interaction stiffness and damping coefficient, after averaging over typically 50 approach curves, is less than $1 \%$ and smaller than the systematic error but of the same order of magnitude. If needed, the deviations presented in the Appendix, can be used as correction factors.

Influence of noise and approach speed - The cantilever displacement recorded as a function time, has been analyzed using both its PSD (in the frequency domain) and its ACF (in the time), leading to identical results within experimental error. The obtained PSDs (see Figs. 3.2 and 3.3) show indeed no spurious resonance peaks as observed in the case of actively driven cantilevers. However, a small white noise background is observed, as well as an excess power at low frequencies $(<10 \mathrm{kHz})$. This low frequency excess noise power is distance-dependent and hence originates only partially from the electronic $1 / f$ noise. A second contribution can be attributed to the finite sampling time in combination with the approach speed of the substrate. During the sampling time for each data block ( $2^{16}$ points), the substrate is displaced over $0.13 \mathrm{~nm}$. This displacement leads to a variation of the tipsubstrate interaction and hence to a variation of the average tip position of at most $0.1 \mathrm{~nm}$ per acquisition, which affects the calculation of the PSD. This effect can be reduced by reducing the sampling time. Experimental tests show indeed a strong reduction of this low frequency excess noise power. However, at the same time the accuracy decreases. These findings were confirmed by numerical simulations. In the time domain the white noise contribution to the signal is only detected at zero time lag while the power excess at low frequencies manifests itself by an offset in the ACF. These findings were also confirmed by numerical simulation. In both approaches, the effects can be fully taken into account, without introducing any systematic error in the 
Chapter 3. Atomic force microscopy of confined liquids using the thermal

analysis.

Comparison with acoustic or magnetic driving - As mentioned above, we observe no spurious resonance in our PSDs, which can hardly be prevented in acoustic actuation [26,33]. Moreover, because the thermal noise covers a broad frequency range it is sufficient to determine the PSD or $\mathrm{ACF}$, circumventing the tedious phase determination as necessary in acoustic or magnetic driving. These spurious resonances and possible errors in the phase determination give rise to an erroneous coupling of the conservative and dissipative contributions in the analysis of the tip-substrate interactions.

Overall we can state that the thermal noise method is more sensitive than methods proposed so far as long as the quality factor is greater than 1 . The detection threshold for the resonance frequency shift is $50 \mathrm{~Hz}$ at $70 \mathrm{kHz}$, while variations in $Q$ of $0.5 \%$ are detectable after averaging over typically 50 approach curves (see Fig. 3.4). For $Q<1$, i.e. for small tip-substrate distances, the method cannot be used. However, by optimizing the cantilever geometry [60], the $Q=1$ condition is reached for smaller distances.

Reynolds damping measurements - Measuring the Reynolds damping in the distance range from 5 to $20 \mathrm{~nm}$ we were able to validate the method as can be concluded from Fig. 3.2. Indeed the expected $d^{-1}$ dependence was found as well as the right bulk viscosity of OMCTS at room temperature if we allow for a small offset in the apparent tip-substrate distance of $0.8 \mathrm{~nm}$. We attribute this offset to a combination of possible local irregularities on the tip surface $[61,62]$ and an uncertainty in our exact 'zero' tip-substrate distance (in contrast to the measurements with the sharp tip, we were unable to image the graphite lattice with these blunt tips). Thus, within the experimental accuracy no slip of the OMCTS on the HOPG substrate is observed in line with complete wetting of OMCTS on HOPG. Alternatively, the offset can be attributed to the existence of an immobile layer on the substrate causing a negative slip-length (the zero-velocity plane is shifted inwards). Negative slip-lengths have been reported in SFA studies on OMCTS confined between two mica sheets $[9,14]$ and can in general be caused by commensurability between the solid and the liquid [63]. Globally, OMCTS and graphite are incommensurate. Yet, it was recently shown in MD simulations that specific chemical interactions [64] or an interaction-induced geometric adjustment of the atoms in the liquid-molecule to the surface [65] can also result in a negative slip length. However, there has been no direct evidence of these effects occurring between OMCTS and graphite [66].

Solvation force and distance dependent dissipation - The results obtained with sharp tips for tip-substrate distances ranging from 2 to $8 \mathrm{~nm}$ show clearly the decaying oscillatory behavior of the stiffness due to the con- 
servative solvation force versus distance. The thermal noise method is significantly more sensitive than earlier measurements using acoustically driven cantilevers [24]. While those earlier measurements revealed excess dissipation only upon squeezing out the last three layers of OMCTS up to a maximum tip-substrate separation of approximately $d=2.4 \mathrm{~nm}$, the current measurements reveal an oscillatory structure in the dissipation up to $d=6 \mathrm{~nm}$ with a total of five local maxima. Yet, the thermal noise method cannot probe the liquid layers in immediate contact with the solid surface due to excessive damping for $d<1.5 \mathrm{~nm}$. According to Fig. 3.3, this implies that the local maxima in the dissipation shown in Fig. 3.5(b) correspond to film thicknesses of 3 to 7 molecular layers. The local maximum at the smallest tip-substrate separation shown in Fig. 3.5(b) (at $d \approx 2.2 \mathrm{~nm}$ ) thus corresponds to the peak at the largest tip-substrate separation in Refs. [24] and [54] at $d \approx 2.4 \mathrm{~nm}$. (The absolute zero of the tip-substrate separation in Ref. [24] is off by approximately $0.6 \mathrm{~nm}$.) This assignment is corroborated by the absolute value of $\gamma_{i n t}$, which is approximately $10^{-6} \mathrm{Ns} / \mathrm{m}$ in both experiments. Comparing the sensitivity of the two experiments reveals a gain of approximately one order of magnitude in the present experiments.

The MD simulations reported in Ref. [54] suggest that the sharp excess dissipation in upon squeezing out the layers in immediate contact with the solid walls is related to confinement-induced transitions between very well ordered (solid-like) films for film thicknesses of one, two, and perhaps three molecular layers and rather disordered configurations for intermediate halfinteger numbers of molecular layers. Whether or not that scenario also extends to the much larger tip-substrate separations studied here is not clear. At larger separations, thermal fluctuations as well as the tip geometry on a larger scale may lead to increased disorder [67-69] and thus prevent the crystalline arrangement found at smaller separations.

\subsection{Conclusion}

In summary, we measured the distance-dependent solvation forces and the dissipation of OMCTS in the confinement between a silicon tip and an HOPG substrate using atomic force microscopy. To obtain reliable results for the distance dependent dissipation in the confinement we employ the thermal noise approach, which provides a resolution of approximately $50 \mathrm{~Hz}$ in frequency shift and $10 \mathrm{nNs} / \mathrm{m}$ in damping coefficient as long as the quality factor is larger than unity, which is the case for tip-substrate distances larger than $2 \mathrm{~nm}$ in the present experiments. To validate the method the distance de- 
Chapter 3. Atomic force microscopy of confined liquids using the thermal 60

bending fluctuations of the cantilever

pendent Reynolds damping and bulk viscosity of OMCTS were successfully measured. Close to the substrate we were able to measure the interaction stiffness due to the solvation forces in agreement with earlier findings, while the damping also showed pronounced oscillations instead of monotonic behavior as a function of tip-substrate distance. From a technical perspective, we presented a method to perform small amplitude force microscopy without relying on a perfect spectral response, in both amplitude and phase, to the external actuation of the cantilever, just by analyzing the thermal noise of the cantilever, which also guarantees small amplitudes of typically $50 \mathrm{pm}$. Moreover, it was shown that the thermal noise can be evaluated equally well with the power spectral density as with the autocorrelation function.

\section{Acknowledgment}

This work has been supported by the Foundation for Fundamental Research of Matter (FOM), which is financially supported by the Netherlands Organization for Scientific Research (NWO). 


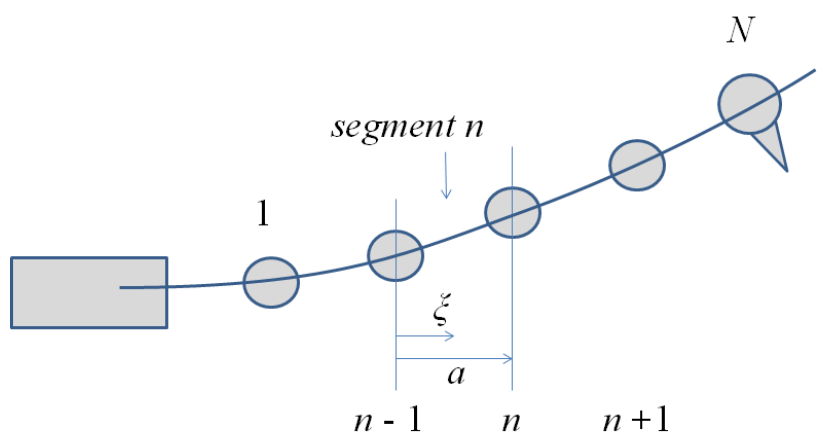

Figure A1: Sketch of the configuration considered.

\section{A Appendix}

\section{A.1 Thermal vibrations of a cantilever beam with tip- substrate interaction}

As stated in Section 3.2.3, we model the cantilever dynamics as a single harmonic oscillator (SHO) with only one resonance frequency. However, due to its distributed mass and friction, the cantilever will show several vibrational bending modes each with its own resonance frequency. In our experimental approach we only consider the frequency response around the lowest (and most prominent) resonance frequency. In this Appendix we will show that in this frequency regime the cantilever behavior deviates only slightly form SHO behavior, also in the presence of an interaction force at the tip of the cantilever. Moreover, we will quantify these deviations in terms of the interaction stiffness and interaction damping as defined in Section 3.2.3, by comparing the full frequency response with that of a best fitting SHO response.

We consider the cantilever as a chain of $N$ distributed masses connected by massless cantilever segments as depicted in Fig. A1. Bead $n$ with mass $m$ exerts a force $B_{n}$ on the cantilever which is equal to:

$$
B_{n}=f_{n}-m \ddot{u}_{n}-\zeta \dot{u}_{n}
$$

where $f_{n}$ is the external (in this case Brownian) force on bead $n, u_{n}$ its displacement and $\zeta$ its friction coefficient. If the tip interacts with the substrate we replace $f_{N}$ by $f_{N}-\left(k_{\text {int }} u_{N}+\left(\gamma_{\text {int }}+\zeta_{\text {tip }}\right) \dot{u}_{N}+m_{\text {tip }} \ddot{u}_{N}\right)$. 
Chapter 3. Atomic force microscopy of confined liquids using the thermal

Assuming $N$ beads on the cantilever, with spacing $a$, we consider a single segment $n$ situated between $m_{n-1}$ and $m_{n}$. Both the total force $F$ and torque $S$ should be zero on this segment:

$$
\begin{aligned}
F_{r}^{[n]}-F_{l}^{[n]} & =0 \\
S_{r}^{[n]}+a F_{r}^{[n]}-S_{l}^{[n]} & =0
\end{aligned}
$$

where $r$ stands for right and $l$ for left.

Since $F_{r}^{[n]}=B_{n}+F_{l}^{[n+1]}$ and $S_{r}^{[n]}=S_{l}^{[n+1]}$ we define:

$$
F_{n}=F_{r}^{[n]} \quad \text { and } \quad S_{n}=S_{r}^{[n]}
$$

and Eq. (a3) reduces to:

$$
S_{n}+a F_{n}=S_{n-1}
$$

The force $F_{n}$ is equal to the sum of all external forces $B_{m}$ acting on the right of segment $n$ :

$$
F_{n}=\sum_{m=n}^{N} B_{m}
$$

while the torque $S_{n}$ is equal to the sum of all external moments $(m-n) a B_{m}$ acting on the right of segment $n$ :

$$
S_{n}=a \sum_{m=n}^{N}(m-n) B_{m} .
$$

Solving the Force and torque balance on segment $n$ one obtains relations for the displacement $u$ and the slope $a d u / d x$ of mass $n$ and $n-1$ :

$$
\begin{aligned}
& u_{n-1}=u_{n}-u_{n}^{\prime}+\frac{S_{n} / a}{2 \kappa}+\frac{F_{n}}{6 \kappa} \\
& u_{n-1}^{\prime}=u_{n}^{\prime}-\frac{S_{n} / a}{\kappa}-\frac{F_{n}}{2 \kappa} \\
& S_{n-1}=S_{n}+a F_{n} \\
& F_{n-1}=F_{n}+B_{n-1}
\end{aligned}
$$

where $\kappa=E I / a^{3}$ and $B_{n}=f_{n}-q u_{n}$ with $q(\omega)=-m \omega^{2}+j \zeta \omega$. With Eqs. (a8-a11) we can calculate all $u_{n}$ and $u_{n}^{\prime}$ values in the frequency domain 
starting from a guess for $u_{N}$ and $u_{N}^{\prime}$ and we will end up with the linear relations:

$$
\begin{aligned}
& u_{0}=A_{0} u_{N}+A_{1} u_{N}^{\prime}+\sum_{n=1}^{N} \alpha_{n} f_{n}, \\
& u_{0}^{\prime}=A_{2} u_{N}+A_{3} u_{N}^{\prime}+\sum_{n=1}^{N} \beta_{n} f_{n} .
\end{aligned}
$$

By inversion of last two equations we can express $u_{N}$ and $u_{N}^{\prime}$ in $u_{0}, u_{0}^{\prime}$ and the forces $f_{n}$ according:

$$
\begin{array}{ll}
u_{N}=\frac{A_{3}}{[A]} u_{0}-\frac{A_{1}}{[A]} u_{0}^{\prime}+\sum_{n=1}^{N} G_{n} f_{n}, & G_{n}=\frac{\beta_{n} A_{1}-\alpha_{n} A_{3}}{[A]} \\
u_{N}^{\prime}=\frac{A_{0}}{[A]} u_{0}^{\prime}-\frac{A_{2}}{[A]} u_{0}+\sum_{n=1}^{N} H_{n} f_{n}, & H_{n}=\frac{\alpha_{n} A_{2}-\beta_{n} A_{0}}{[A]}
\end{array}
$$

with $[A]=A_{0} A_{3}-A_{1} A_{2}$. The coefficients $\alpha_{n}, \beta_{n}$ and $A_{n}$ can be calculated by evaluating Eqs. (a8-a11) while setting one of the values $\left(u_{N}, u_{N}^{\prime}, f_{1}, f_{2}, . ., f_{N}\right)$ equal to one and keeping all the other values zero. Since we consider the noise response we set both $u_{0}$ and $u_{0}^{\prime}$ to zero for all frequencies. Because the power spectral density (PSD) of the Brownian forces is constant, i.e. $P_{f}(\omega)=2 \zeta k_{B} T$, the power spectral density $P_{s}(\omega)$ of the signal $u_{N}^{\prime}(\omega) / a$ is given by (no interaction on the tip):

$$
P_{s}(\omega)=\frac{1}{a^{2}} \sum_{n=1}^{N}\left|H_{n}(\omega)\right|^{2} P_{f_{n}}(\omega)=\frac{2 k_{B} T}{L^{2}} N^{2} \sum_{n=1}^{N} \zeta_{n}\left|H_{n}(\omega)\right|^{2} .
$$

Taking into account the interaction force (and additional tip mass) on the tip in the expression for $f_{N}$, we replace it by $f_{N}-\left(k_{\text {int }}+j \omega \gamma_{\text {int }}-m_{\text {added }} \omega^{2}\right) u_{N}$. Hence, we obtain for our signal $u_{N}^{\prime}$ :

$$
\begin{aligned}
u_{N}^{\prime} & =\sum_{n=1}^{N} H_{n} f_{n}-H_{N}\left(k_{\mathrm{int}}+j \omega \gamma_{\mathrm{int}}\right) u_{N} \\
& =\sum_{n=1}^{N} H_{n}^{[\mathrm{int}]} f_{n}
\end{aligned}
$$

with

$$
H_{n}^{[\mathrm{int}]}=H_{n}-\frac{H_{N}\left(k_{\mathrm{int}}+j \omega \gamma_{\mathrm{int}}\right)}{1+G_{N}\left(k_{\mathrm{int}}+j \omega \gamma_{\mathrm{int}}\right)} G_{n}
$$


Chapter 3. Atomic force microscopy of confined liquids using the thermal 64 bending fluctuations of the cantilever

Because the interaction damping also modifies the random force on the tip: $P_{f_{N}}(\omega)=2 k_{B} T\left(\zeta_{N}+\gamma_{\text {int }}\right)$, the power spectral density of our signal becomes:

$$
P_{s}(\omega)=\frac{2 k_{B} T}{a^{2}}\left(\left(\zeta_{N}+\gamma_{\mathrm{int}}\right)\left|H_{N}^{[\mathrm{int}]}(\omega)\right|^{2}+\zeta \sum_{n=1}^{N-1}\left|H_{n}^{[\mathrm{int}]}(\omega)\right|^{2}\right)
$$

To evaluate Eq. (a18-a19) numerically, we define a reference frequency $\omega_{\#}=\sqrt{k_{c} / m_{\text {tot }}}$, where $k_{c}=3 E I / L^{3}$. Than we get, with $\nu=\omega / \omega_{\#}$, for the relevant variables:

$$
\begin{aligned}
q & =-m \omega_{\#}^{2} \nu^{2}+j \zeta \omega_{\#} \\
m & =\alpha m_{\mathrm{tot}} / N \\
m_{N} & =(1-\alpha+\alpha / N) m_{\mathrm{tot}} \\
\zeta & =\alpha \zeta_{\mathrm{tot}} / N \\
\zeta_{N} & =(1-\alpha+\alpha / N) \zeta_{\mathrm{tot}}
\end{aligned}
$$

where $(1-\alpha) m_{\text {tot }}$ is the mass of the tip. With $Q^{-1}=\zeta_{\text {tot }} / \sqrt{m_{\text {tot }} k_{c}}$ we arrive at:

$$
\begin{aligned}
q & =\frac{\alpha k_{c}}{N}\left(-\nu^{2}+j \nu / Q\right) \\
q_{N} & =\left(\frac{\alpha}{N}+1-\alpha\right) k_{c}\left(-\nu^{2}+j \nu / Q\right)
\end{aligned}
$$

and

$$
P_{s}(\nu)=\frac{2 k_{B} T}{L^{2}} N\left\{\alpha \zeta_{\text {tot }} \sum_{n=1}^{N}\left|H_{n}^{[\mathrm{int}]}\right|^{2}+\left((1-\alpha) N \zeta_{\text {tot }}+\gamma_{\text {int }}\right)\left|H_{N}^{[\mathrm{int}]}\right|^{2}\right\}
$$

where $H_{n}^{[\mathrm{int}]}$ is a function of the complex quantity $\left[k_{\text {int }}+j Q^{-1}\left(k_{c} / \zeta_{\text {tot }}\right) \gamma_{\text {int }} \nu\right]$, see Eq. (a18). We evaluated Eq. (a27) numerically varying $k_{\text {int }} / k_{c}$ and $\gamma_{\text {int }} / \zeta_{\text {tot }}$, and fitted the obtained $P_{s}(\nu)$ curves to the SHO model. This resulted in good fits with a low chi square per data point. From these fits the values for $k_{\text {int }} / k_{c}$ and $\gamma_{\text {int }} / \zeta_{\text {tot }}$ were recalculated and compared with their original values. The systematic errors $\Delta k_{\text {int }}=k_{\text {int }}^{\text {fit }}-k_{\text {int }}$ and $\Delta \gamma_{\text {int }}=$ $\gamma_{\text {int }}^{\text {fit }}-\gamma_{\text {int }}$ have been plotted in Figs. A2 and A3, where $k_{\text {int }}$ has been scaled on $k_{c}$ and $\gamma_{\text {int }}$ on $\zeta_{\text {tot }}$. 


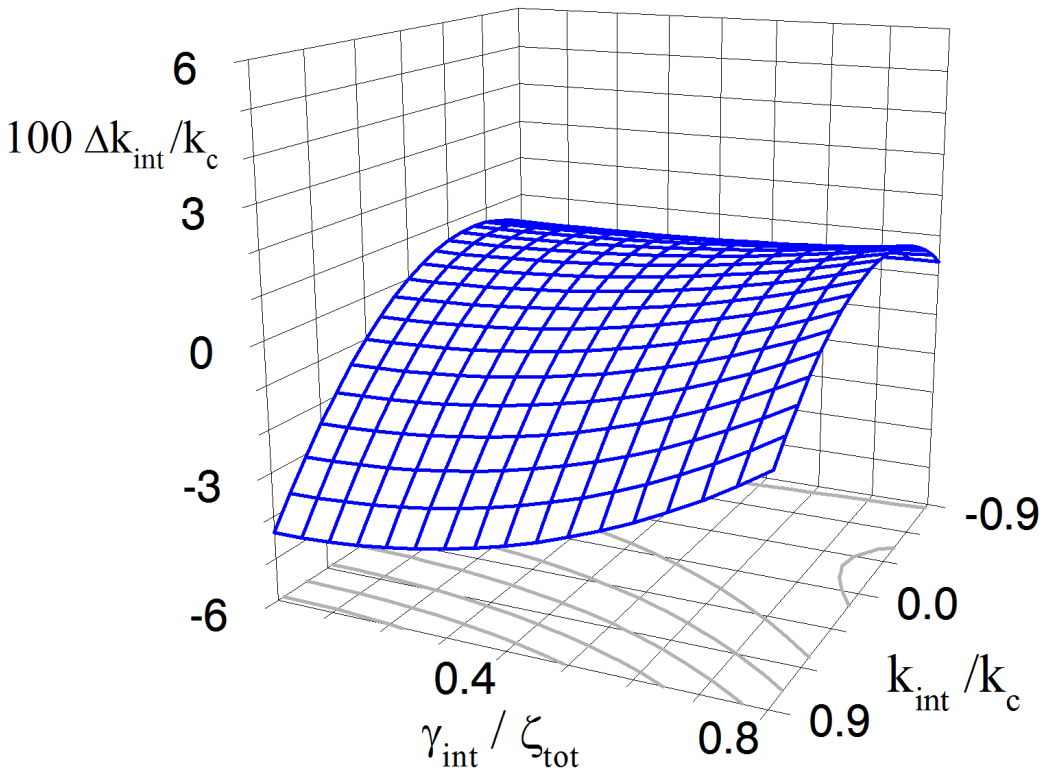

Figure A2 $\Delta k_{\text {int }}$ vs $\left(k_{\text {int }}, \gamma_{\text {int }}\right)$ for $N=32$ and $\alpha=1.0$. Note the vertical scale is blown up with a factor $100 . k_{\text {int }}$ is taken relative to $k_{c}=3 E I / L^{3}$. The systematic error in $k_{\text {int }}$ is in all cases less than $4 \%$ of $k_{c}$. 
Chapter 3. Atomic force microscopy of confined liquids using the thermal 66

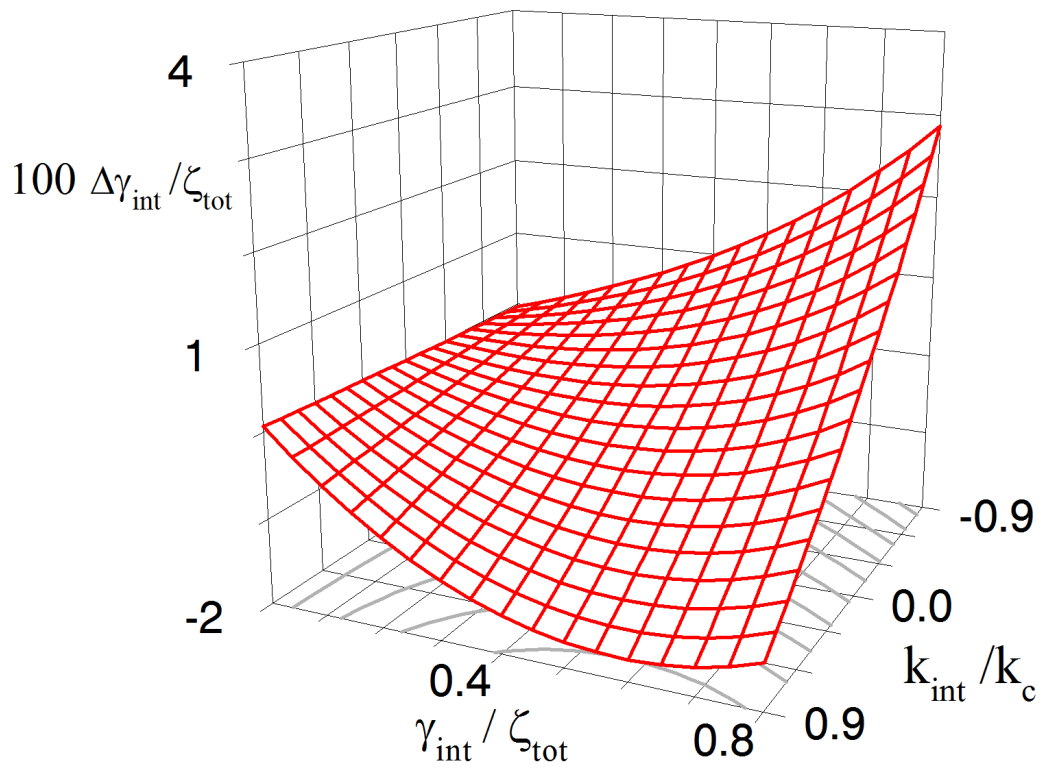

Figure A3 $\Delta \gamma_{\text {int }}$ vs $\left(k_{\text {int }}, \gamma_{\text {int }}\right)$ for $N=32$ and $\alpha=1.0$. Note the vertical scale is blown up with a factor $100 . \gamma_{\text {int }}$ is taken relative to $\zeta_{\text {tot }}$. The systematic error in $\gamma_{\text {int }}$ is in all cases less than $3 \%$ of $\zeta_{\text {tot }}$.

\section{A.2 Supplementary Information}

Fit the measured interaction stiffness to van der Waals interaction

The interaction stiffness is measured with a silicon tip $\left(R_{t i p}=900 \mathrm{~nm}\right.$, see the inset of Fig. 3.2(b)). The system is modeled as a graphite semi-finite wall and a spherical silicon particle with a native oxidized layer in the medium of OMCTS. The averaged data is fitted to van der Waals interaction as expressed in

$$
k_{\text {int }}(d)=-\frac{A_{\text {sio }}-R_{t i p}}{3 d^{3}}-\frac{\left(A_{s i}-A_{\text {sio }}\right) R_{\text {tip }}}{3(d+h)^{3}}+\Delta k
$$

where the silica layer thickness $h$ and the offset $\Delta k$ are parameters to fit. The offset is to account for the deviation from the assumption that at the 


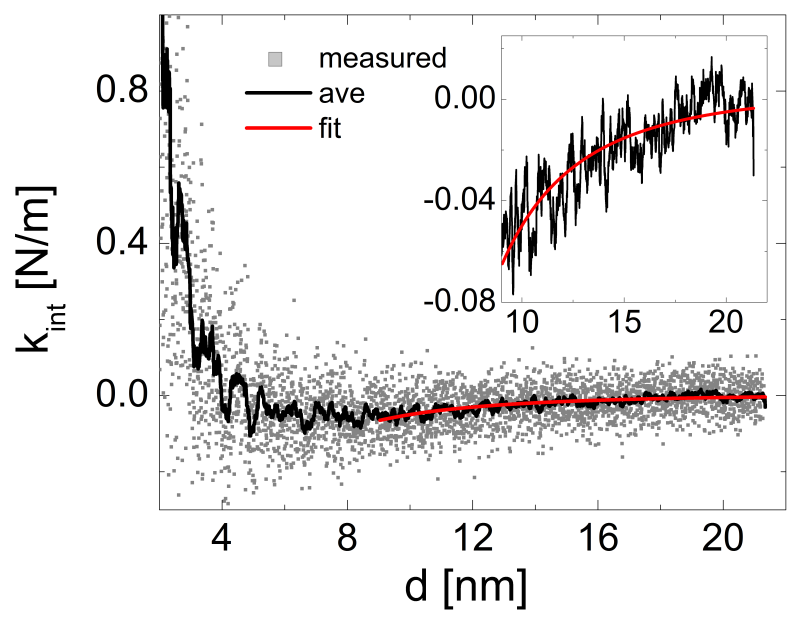

Figure S1: Measured interaction stiffness (measured data points in gray dots, smoothed in black line) and the fit of van der Waals interaction (red) from $d>9 \mathrm{~nm}$. The inset is a zoom-in of the smoothed data and fit curve.

furthest measured distance the interaction stiffness is negligible at $d=21 \mathrm{~nm}$ when we extract the interaction stiffness. The Hamaker constants of silicon and silica are $A_{s i}=3.49 \times 10^{-19} \mathrm{~J}$ and $A_{\text {sio }}=3.18 \times 10^{-20} \mathrm{~J}$ respectively. The averaged interaction stiffness from $d>9 \mathrm{~nm}$ is fitted by the red line in Fig. S1. The fitted $h$ is $2.89 \pm 0.06 \mathrm{~nm}$, which agrees with typical thickness of native silica on silicon. The fitted $\Delta k$ is very small, $0.0042 \pm 0.0003 \mathrm{~N} / \mathrm{m}$, i.e. $<0.22 \%$ of $k_{c}$, which justifies our assumption during extracting interaction stiffness - the interaction stiffness at $21 \mathrm{~nm}$ is zero.

\section{Distribution of offsets}

From one sharp tip, 97 approach curves are analyzed. As stated in the result section, the individual curves were aligned by shifting the two peak positions at $\sim 2.4 \mathrm{~nm}$ and $\sim 3.3 \mathrm{~nm}$ in the resonance frequency profile (see Fig. 3.4) on top of each other to compensate for residual drift. The offset distances are plotted in the histogram below. 
Chapter 3. Atomic force microscopy of confined liquids using the thermal 68 bending fluctuations of the cantilever

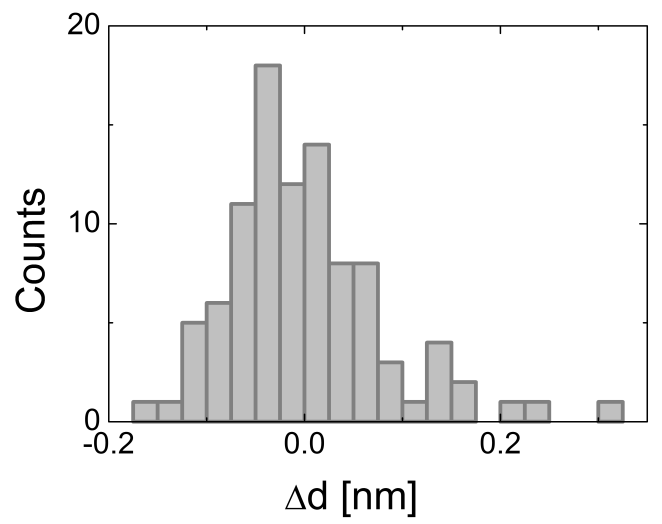

Figure S2: Histogram of offset distances for aligning extracted results from 97 approaches. 


\section{Bibliography}

[1] Bocquet L and Charlaix E 2010 Chem. Soc. Rev. 391073

[2] Eijkel J C T and van den Berg A 2005 Microfluid. Nanofluid. 1249

[3] Persson B N J and Mugele F 2004 J. Phys.: Condens. Matter 16 R295

[4] M. H. Müser,Urbakh M, and Robbins M O 2003 Adv. Chem. Phys. 126 187

[5] Reynolds O 1886 Phil. Trans. R. Soc. London 177157

[6] Chan D Y C and Horn R G 1985 J. Chem. Phys. 835311

[7] Mugele F and Salmeron M 2000 Phys. Rev. Lett. 845796

[8] Israelachvili J N 1991 Intermolecular and Surface Forces 2nd edition (Academic press, London)

[9] Horn R G and Israelachvili J N 1980 Chem. Phys. Lett. 71192

[10] O'Shea S J and Welland M E 1998 Langmuir 144186

[11] Antognozzi M, Humphris A D L, and Miles M J 2001 Appl. Phys. Lett. 78300

[12] Kageshima M, Jensenius H, Dienwiebel M, Nakayama Y, Tokumoto H, Jarvis S P, and Oosterkamp T H 2002 Appl. Surf. Sci. 188440

[13] Zhu Y and Granick S 2003 Langmuir 198148

[14] Becker T and Mugele F 2003 Phys. Rev. Lett. 91166104

[15] Ashby P D and Lieber C M 2004 J. Am. Chem. Soc. 12616973

[16] Maali A, Hurth C, Cohen-Bouhacina T, Couturier G, and Aimé J-P 2006 Appl. Phys. Lett. 88163504

[17] Maali A, Cohen-Bouhacina T, Couturier G, and Aimé J-P 2006 Phys. Rev. Lett. 96086105

[18] Patil S, Matei G, Oral A, and Hoffmann P M 2006 Langmuir 226485

[19] Kaggwa G B, Kilpatrick J I, Sader J E, and Jarvis S P 2008 Appl. Phys. Lett. 93011909

[20] Bureau L and Arvengas A 2008 Phys. Rev. E 78061501 
[21] Hofbauer W, Ho R J, Hairulnizam R, Gosvami N N, and O'Shea S J 2009 Phys. Rev. B 80134104

[22] Bureau L 2010 Phys. Rev. Lett. 104218302

[23] Khan S H, Matei G, Patil S, and Hoffmann P M 2010 Phys. Rev. Lett. 105106101

[24] de Beer S, van den Ende D, and Mugele F 2010 Nanotechnology 21 325703

[25] de Beer S, van den Ende D, and Mugele F 2011 J. Phys.: Condens. Matter 2311206

[26] Kiracofe D and Raman A 2011 Nanotechnology 22485502

[27] Labuda A , Kobayashi K, Suzuki K, Yamada H, and P Grütter 2013 Phys. Rev. Lett. 110066102

[28] Gee M L, McGuiggan P M, Israelachvili J N, and Homola A M $1990 \mathrm{~J}$. Chem. Phys. 931895

[29] Klein J and Kumacheva E 1995 Science 269816

[30] Schindler M 2010 Chem. Phys. 375327

[31] Labuda A and Grütter P 2012 Langmuir 285319

[32] Li T D and Riedo E 2008 Phys. Rev. Lett. 100106102

[33] Schäffer T E, Cleveland J P, Ohnesorge F, Walters D A, and Hansma P K 1996 J. Appl. Phys. 803622

[34] Asakawa H and Fukuma T 2009 Rev. Sci. Instrum. 80103703

[35] Carrasco C, Ares P, de Pablo P J, and Gómez-Herrero J 2008 Rev. Sci. Instrum. 79126106

[36] de Beer S, van den Ende D, and Mugele F 2008 Appl. Phys. Lett. 93 253106

[37] Revenko I and Proksch R 2000 J. Appl. Phys. 87526

[38] Xu X, Koslowski M, and Raman A 2012 J. Appl. Phys. 111054303

[39] Herruzo E T and Garcia R 2007 Appl. Phys. Lett. 91143113

[40] O'Shea S J 2006 Phys. Rev. Lett. 97179601

[41] Sader J E and Jarvis S P 2006 Phys. Rev. B 74195424

[42] Hutter J L and Bechhoefer J 1993 Rev. Sci. Instrum. 641868

[43] Willemsen O H, Kuipers L, van der Werf K O, de Grooth B G, and Greve J 2000 Langmuir 164339

[44] Cleveland J P, Schäffer T E, and Hansma P K 1995 Phys. Rev. B 52 $\mathrm{R} 8692$

[45] Benmouna F and Johannsmann D 2003 J. Phys.: Condens. Matter 15 3003 
[46] Roters A, Gelbert M, Schimmel M, Rühe J, and Johannsmann D 1997 Phys. Rev. E 563256

[47] von Sicard O, Gigler A M, Drobek T, and Stark R W 2009 Langmuir 252924

[48] Ulcinas A, Valdre G, Snitka V, Miles M J, Claesson P M, and M Antognozzi 2011 Langmuir 2710351

[49] Kubo R Rep. Prog. Phys. 29255

[50] Labuda A, Lysy M, and Grütter P 2012 Appl. Phys. Lett. 101113105

[51] Wiener N 1930 Acta Math. 55117

[52] Khintchine A 1934 Math. Ann. 109604

[53] Würger A 2011 J. Phys.: Condens. Matter 23505103

[54] de Beer S, den Otter W K, van den Ende D, Briels W J, and Mugele F 2012 Europhys. Lett. 9746001

[55] Borkent B M, de Beer S, Mugele F, and Lohse D 2010 Langmuir 26260

[56] Norrelykke S F and Flyvbjerg H 2011 Phys. Rev. E 83041103

[57] Klein J 2007 Phys. Rev. Lett. 98056101

[58] Cottin-Bizonne C, Cross B, Steinberger A, and Charlaix E 2005 Phys. Rev. Lett. 94056102

[59] E Sader J 1998 J. Appl. Phys. 8464

[60] Katan A J and Oosterkamp T H 2008 J. Phys. Chem. C 1129769

[61] O'Shea S J 2001 Jpn. J. Appl. Phys. 404309

[62] Khan S H and Hoffmann P M arXiv:1210.3540.

[63] P. A Thompson and M. O. Robbins. Phys. Rev. A, 41:6830, 1990.

[64] Kong L-T, Denniston C, and Müser M H 2010 Modelling Simul. Mater. Sci. Eng. 18034004

[65] Vadakkepatt A, Dong Y, Lichter S, and Martini A Phys. Rev. E 84 066311

[66] de Beer S, Wennink P, van der Weide-Grevelink M, and Mugele F 2010 Langmuir $\mathbf{2 6} 13245$

[67] Luan B Q and Robbins M O 2005 Nature 435929

[68] Mo Y F, Turner K T, and Szlufarska I 2009 Nature 4571116

[69] de Beer S, den Otter W K, van den Ende D, Briels W J, and Mugele F Tribol. Lett. 481 



\section{Chapter 4}

\section{Amplitude modulation Atomic Force Microscopy, is acoustic driving in liquid quantitatively reliable?}

Measuring quantitative tip-sample interaction forces in dynamic Atomic Force Microscopy in fluids is challenging because of the strong damping of the ambient viscous medium and the fluid-mediated driving forces. This holds in particular for the commonly used acoustic excitation of the cantilever oscillation. Here we present measurements of tip-sample interactions due to conservative DLVO and hydration forces and viscous dissipation forces in aqueous electrolytes using tips with radii varying from typical $20 \mathrm{~nm}$ for the DLVO and hydration forces, to $1 \mu \mathrm{m}$ for the viscous dissipation. The measurements are analyzed using a simple harmonic oscillator model, continuous beam theory with fluid-mediated excitation and thermal noise spectroscopy (TNS). In all cases consistent conservative forces, deviating less than $40 \%$ from each other, are obtained for all three approaches. The DLVO forces are even within $5 \%$ of the theoretical expectations for all approaches. Accurate measurements of dissipative forces within $15 \%$ of the predictions of macroscopic fluid dynamics require the use of TNS or continuous beam theory including fluid-mediated driving. Taking this into account, acoustic driving in liquid is quantitatively reliable. 


\subsection{Introduction}

In various disciplines of bioscience and nanoscience, one studies phenomena on a nano scale, and tries to reveal the microstructure of the sample at hand or to probe the strength of these structures. Atomic Force Microcopy (AFM) is a versatile tool for high-resolution imaging and nanoscopic force spectroscopy [1-3]. It can be operated in several modes, such as contact mode and non-contact mode, both used for imaging. To measure tip substrate interactions one usually applies amplitude modulation (AM) or frequency modulation (FM) in non-contact mode $[4,5]$. For this several actuation schemes have been developed, such as magnetic actuation $[6,7]$, acoustic or piezo excitation [8] and photo thermal excitation [9, 10]. Among them, photo thermal excitation is very promising, mainly because the force analysis is straight forward and reliable, but it is not yet widely available. Instead, amplitude modulation with acoustic excitation is still most widely used because it is easy to implement and it is relatively cheap. In this case the cantilever is acoustically driven by a piezo stack to which the cantilever base has been attached. For force spectroscopy, amplitude and phase of the tip deflection are measured. From this deflection the tip-sample interaction forces can be obtained, for instance by modeling the tip of the cantilever as a simple harmonic oscillator ( $\mathrm{SHO}$ ). This procedure is called force inversion. For application under vacuum or ambient air, the cantilever dynamics is well established. In contrast, when applied in liquid, the dynamics are more complicated and its analysis is still under development. In vacuum, the quality factor $Q$ of the oscillator is generally larger than 1000. In liquids, due to hydrodynamic loading, $Q$ is usually less than 10. As a result, the base motion amplitude becomes comparable to that of the cantilever tip oscillations $[7,11,12]$. So the tip position is no longer proportional to the measured deflection, but depends also on the base displacement. A complicating factor is the unknown phase and amplitude of the base motion with respect to the drive signal of the piezo. Under liquid the resonance peak in the piezo-response spectrum of the cantilever itself is often obscured by resonances due to the piezo, the surrounding fluid and the geometry, which causes the 'forest of peaks' often observed in acoustic driving $[13,14]$. See Fig. 4.1(b) for an example.

Recently, Raman and coworkers [14] showed that this forest of peaks is caused by the frequency dependence of the amplitude and phase of the base motion. This is in contradiction with the common assumption that the base motion is just proportional to the electric driving signal with a frequency 
(a)

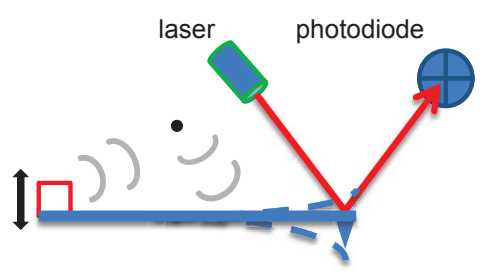

(b)

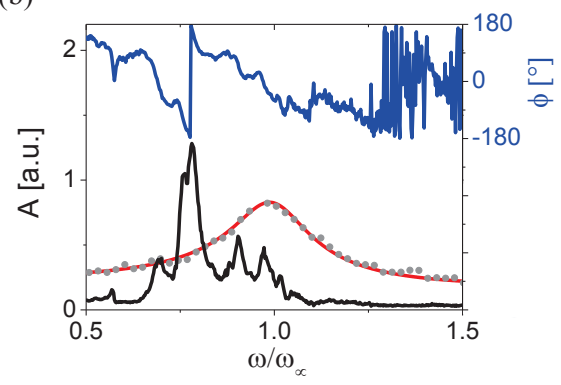

Figure 4.1: (a) Principle of AM-AFM force spectroscopy with piezo excitation and optical deflection detection. The piezo also excites the cantilever via the surrounding liquid. (b) Amplitude (black curve) and phase (red curve) of the response function of a cantilever and its thermal spectrum; circles indicate experimental values, the line represents the best fitting SHO curve.

independent proportionality constant. By considering the ratio of the cantilever deflection $u_{t}$ and its base motion $z_{b}$ one can correct for this omission. However, the calculated response $u_{t} / z_{b}$ is generally considerably smaller than the experimentally obtained response. Again Raman and coworkers offered an explanation: because the surrounding liquid is also excited by the piezo, the cantilever is driven via the liquid, too [7,14] (see Fig. 4.1(a) for an artists impression). They incorporated this secondary excitation into their analysis, which asks for a full continuous beam analysis of the vibrating cantilever [14]. As amplitude and phase of the base motion are not known, nor the excitation constant of the liquid mediated piezo driving, the measured amplitude and phase of the cantilever deflection cannot directly be related to the interaction forces. Besides a calibration of the resonance frequency and quality factor of the cantilever under liquid, also a calibration procedure is required to determine the just mentioned unknowns. All these aspects are often overlooked 

is acoustic driving in liquid quantitatively reliable?

when one uses the formulas of force inversion developed for AFM in vacuo. This leads sometimes to contradictory results, specially in the observed dissipation. For example, the forces measured in nano-confined liquids have been debated intensively during the past decade [15]. Such discrepancies were commonly attributed to differences in sample preparation and/or the geometry of the probe apex, but can be attributed as well to this secondary driving mechanism. Including the contribution of Raman and coworkers several models, with different levels of complexity, are proposed to describe the cantilever dynamics in AM-AFM. However, a quantitative validation of these approaches is still missing. This motivates us to compare them for a number of well characterized tip-substrate interactions with both the thermal noise spectroscopy method [16] and theoretical models of these interactions.

In a previous investigation, we explored thermal noise spectroscopy (TNS) and demonstrated its abilities when performing force inversion [16]. With TNS the cantilever position only fluctuates due to thermal agitation by the surrounding fluid. The interaction forces are extracted from the shift of the resonance peak and the change in peak width in the power spectral density (PSD) distribution due to these interactions. Hence, we eliminate the aforementioned effects (unknown base motion, fluid mediated piezo driving) from the force inversion procedure. But this approach has also some limitations. It is not applicable when the cantilever motion is overdamped, which is frequently encountered when the tip is in close vicinity of the substrate $(d \leq 1$ $\mathrm{nm}$ ). Moreover, since the driving force is stochastic, relatively long time sequences are required to get an accurate PSD. It typically takes a fraction of a second to obtain a statistically reliable spectrum, and several seconds for a full force distance curve. Hence, the technique is intrinsically slow. Thus, although TNS provides the arguably cleanest and most reliable force inversion procedure, it is not able to substitute force spectroscopy with active excitation in all cases.

In this investigation we focus on the validation of AM force spectroscopy using acoustic excitation, by measuring three different types of forces. The results obtained from AM measurements are directly compared to TNS data as obtained in the same experiment. The first experiment is the determination of the interaction forces [17] between a silica tip and a silica substrate in close proximity in an electrolyte environment. To quantify the accuracy of both approaches, these measurements are compared with theoretical calculations of the DLVO forces, taking into account charge regulation at the silica substrate $[18,19]$. We also investigate the hydrodynamic dissipation, when a colloidal probe approaches a silica substrate, because in case of measuring dissipative forces different researchers observe different behavior while they 
are rather unanimous about the conservative interactions [11,20]. Again we compare the results of these experiments with a theoretical calculation of the Reynolds damping. At last we measure the oscillatory hydration forces between the probe tip and an atomic smooth mica substrate. These hydration force measurements have been debated intensively during the past decade [21-25], but are quantitatively not well understood yet. In the force inversion analysis of these experiments, we consider both the SHO approach and the recently proposed continuous beam approach.

This chapter is organized as follows: first we review the force spectroscopy under study in Section 4.2. Next, we discuss in Section 4.3 the experimental details and the data analysis. In Section 4.4 we show the extracted forcedistance curves as obtained with the different methods and compare them with our theoretical calculations. In Section 4.5 we discuss our results and eventually, and in Section 4.6 we draw our conclusions.

\subsection{Principles of force spectroscopy}

In this section, we review the principles of AFM force spectroscopy using acoustic actuation, both from an SHO perspective $[11,12]$ and using a continuous beam approach with some recently proposed modifications [14]. We also briefly discuss TNS as developed in an earlier investigation [16].

\subsubsection{Small amplitude modulation with piezo excitation}

In the single harmonic oscillator ( $\mathrm{SHO}$ ) approximation an acoustically driven AFM cantilever, interacting with a substrate, is described by the equation of motion:

$$
m_{c} \ddot{z}=k_{c}\left(z_{b}-z\right)+F_{\text {drag }}+F_{\text {int }}
$$

where $z$ is the momentary displacement with respect to the average interaction distance $d, m_{c}$ the mass of the cantilever and $k_{c}$ its stiffness, while $z_{b}(t)$ is the displacement of the base of the cantilever. The drag force on a sphere with radius $a$ oscillating with frequency $\omega$ in a liquid with density $\rho$ and viscosity $\eta$, is given by $F_{\text {drag }}=-\gamma_{c} \dot{z}-m_{\text {add }} \ddot{z}$, where $\gamma_{c}=6 \pi \eta a+3 \pi a^{2}(2 \eta \rho \omega)^{1 / 2}$ is the effective damping of the cantilever and $m_{\text {add }}=\frac{2}{3} \pi a^{3} \rho+3 \pi a^{2}(2 \eta \rho / \omega)^{1 / 2}$ the added mass due to the surrounding liquid [26]. Moreover, the interaction force is modeled as $F_{\text {int }}=F_{0}(d)-k_{\text {int }} z-\gamma_{\text {int }} \dot{z}$ with $k_{\text {int }}$ the interaction stiffness and $\gamma_{\text {int }}$ the interaction damping. After substitution of both expressions for the forces in Eq. (4.1), this differential equation transforms in the 

is acoustic driving in liquid quantitatively reliable?

frequency domain into an algebraic equation:

$$
\left(k_{c}-G^{*}(\omega)+K^{*}(\omega)\right) \tilde{z}=k_{c} \tilde{z}_{b}
$$

where $\tilde{z}(\omega)$ is the Fourier transform of $z(t), G^{*}(\omega)=\omega^{2} m_{\mathrm{eff}}-j \omega \gamma_{c}$ and $K^{*}(\omega)=k_{\text {int }}+j \omega \gamma_{\text {int }}$ with $m_{\text {eff }}=m+m_{\text {add }}$ and $j$ the imaginary unit $\left(j^{2}=-1\right)$. However, according to Kiracofe and Raman [14] the piezo drives the cantilever not only via the cantilever holder but also indirectly via the surrounding liquid which is not stagnant anymore. Therefore they propose to rewrite the interaction $\gamma_{c} \dot{z}+m_{\text {add }} \ddot{z}$ with the liquid as $\gamma_{c}\left(\dot{z}-v_{f}\right)+m_{\text {add }}\left(\ddot{z}-\dot{v}_{f}\right)$. Here $v_{f}(t)=\int_{0}^{\infty}-B_{f}\left(t^{\prime}\right) \dot{z}_{b}\left(t-t^{\prime}\right) d t^{\prime}$ is the background fluid velocity at the tip. It depends linearly on the base position history. Implementing these changes Eq. (4.2) becomes:

$$
\left(k_{c}-G^{*}+K^{*}\right)\left(\tilde{u}_{t}+\tilde{z}_{b}\right)=\left(k_{c}+G^{*} B_{f}^{*}-m_{c} \omega^{2} B_{f}^{*}\right) \tilde{z}_{b}
$$

where $B_{f}^{*}(\omega)$ is the Fourier transform of $B_{f}(t)$. We replaced $\tilde{z}$ by $\tilde{u}_{t}+\tilde{z}_{b}$, because in an AFM experiment not the position $\tilde{z}$ but the deflection $\tilde{u}_{t}=$ $A e^{j \phi}=\tilde{z}-\tilde{z}_{b}$ is measured. The term $\omega^{2} m_{c} B_{f}^{*}$ will be neglected, because $m_{c} \ll m_{\text {eff }}$ for frequencies near the resonance frequency of the cantilever. Thus:

$$
\frac{\tilde{u}_{t}}{\tilde{z}_{b}}=\frac{\left(1+B_{f}^{*}\right) G^{*}-K^{*}}{k_{c}-G^{*}+K^{*}}
$$

Away from the substrate, where $K^{*}=0$, we obtain:

$$
\frac{\tilde{u}_{t}^{\infty}}{\tilde{z}_{b}}=\frac{\left(1+B_{f}^{*}\right) G^{*}}{k_{c}-G^{*}}
$$

The coefficients $m_{\mathrm{eff}}, k_{c}, \gamma_{c}$ are obtained by measuring the power spectral density in air and under liquid, away from the substrate in a separate procedure called thermal calibration. So we can determine the interaction parameters $k_{\text {int }}=K^{\prime}$ and $\gamma_{\text {int }}=K^{\prime \prime} / \omega$ using Eq. (4.4) and (4.5) without explicit knowledge about $\tilde{z}_{b}$ :

$$
K^{*}=\frac{C\left(1+B_{f}^{*}\right) G^{*}\left(k_{c}-G^{*}\right)(1-\hat{A})}{C\left(1+B_{f}^{*}\right) G^{*} \hat{A}+k_{c}-G^{*}}
$$

where $C=C_{\text {sho }}=1$ and $\hat{A}=\tilde{u}_{t} / \tilde{u}_{t}^{\infty}=\left(A / A_{\infty}\right) e^{j\left(\phi-\phi_{\infty}\right)}$. If fluid mediated driving is not taken into account, i.e $B_{f}^{*}=0$, this approach is equivalent to the treatment in ref. [11] and [12]. If $\tilde{u}_{t} \ll \tilde{z}_{b}$, one can neglect $\tilde{z}_{b}$ in the left 


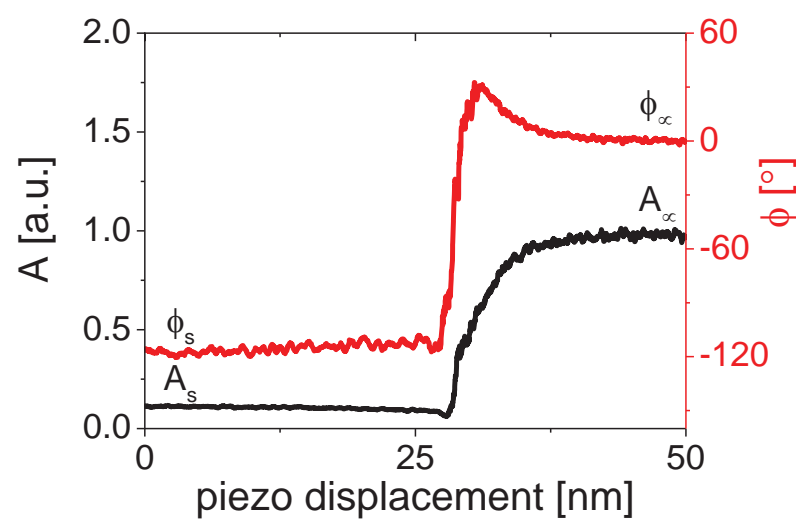

Figure 4.2: AM approach curves for calibration purposes. At large piezo distance $A_{\infty}$ and $\phi_{\infty}$ are determined while for small piezo distances when the tip is in hard contact with the substrate, $A_{s}$ and $\phi_{s}$ are determined.

hand side of Eq. (4.3) and Eq. (4.6) transforms to $K^{*}=\left(k_{c}-G^{*}\right)(1-\hat{A}) / \hat{A}$. In this form it is mostly used for analyzing acoustic driving in air or in vacuo.

In general, to determine $K^{*}$ we first need to fix a value for $B_{f}^{*}$. A reference measurement away from the substrate is not enough anymore to determine the unknown parameters $\tilde{z}_{b}$ and $B_{f}^{*}$. Kiracofe and Raman [14] propose to use also the deflection measurement when the tip is in hard contact with the substrate. To obtain $B_{f}^{*}$ from this measurement, one must keep in mind that the damping does not take place only at the tip but along the whole cantilever. So one has to consider the cantilever shape oscillations $\tilde{z}(x, \omega)$, where $x$ is the coordinate along the beam. These shape oscillations can be decomposed in several normal modes each with its characteristic shape $\psi_{n}(x)$ and resonance frequency $\omega_{n}$. If the cantilever is driven near the resonance frequency of the lowest mode, contributions from the higher modes can be neglected and again Eq. (4.6) can be derived but in this case with $C=C_{\text {cbm }}$, given by:

$$
C_{\mathrm{cbm}}=\psi_{1}(L)\left\langle\psi_{1}\right\rangle /\left\langle\psi_{1}^{2}\right\rangle \simeq 1.57
$$

Here the average has been defined as $\langle f\rangle=L^{-1} \int_{0}^{L} f(x) d x$. This modification of the constant $C$ takes into account the friction and mass distributed along the cantilever beam (see S 1 of the Suppl. Info). To obtain an expression for the constant $B_{f}^{*}$ we follow Raman and coworkers and consider the shape function $\chi_{1}(x)$ with resonance frequency $\bar{\omega}_{1}$ in case the tip is in hard contact 


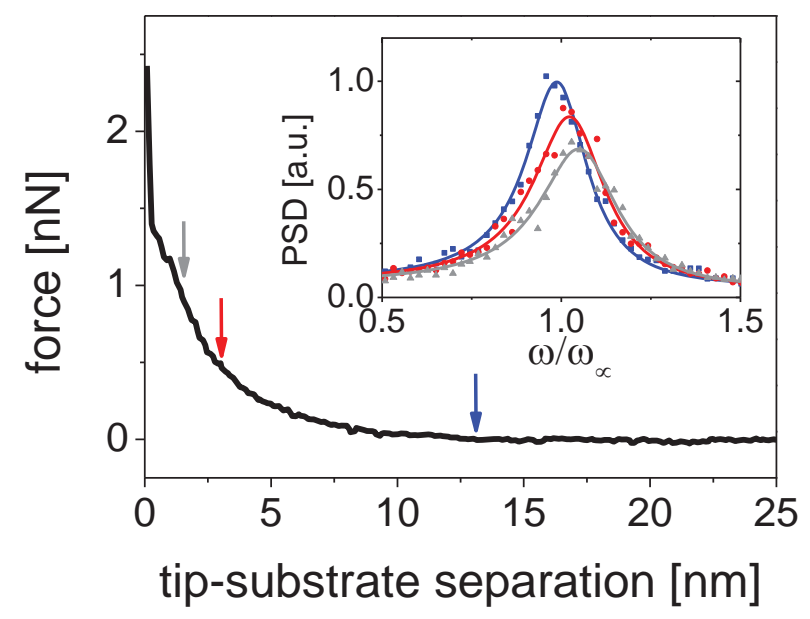

Figure 4.3: A typical approach curve as used in TNS. The inset shows the three spectra generated from the fluctuating deflection signal (symbols) at 3 distances along the force curve, identified by arrows with a matching color, as well as their fits to Eq. (4.9) (lines).

with the substrate. If we define $\tilde{u}_{t}^{s}=A_{s} e^{j \phi_{s}}$ as the tip deflection when it is in hard contact with the substrate, $\bar{k}_{c}=k_{c}\left(\bar{\omega}_{1} / \omega_{1}\right)^{2}, c_{1}=\left\langle\psi_{1}\right\rangle /\left\langle\psi_{1}^{2}\right\rangle$, $\bar{c}_{1}=\left\langle\chi_{1}\right\rangle /\left\langle\chi_{1}^{2}\right\rangle$ and $g_{1}=\left\langle g \chi_{1}\right\rangle /\left\langle\chi_{1}^{2}\right\rangle$ where $g(x)=1-\frac{3}{2}(x / L)^{2}+\frac{1}{2}(x / L)^{3}$ (see Suppl. Info), we obtain for $B_{f}^{*}$ :

$$
B_{f}^{*}=-\frac{\left(k_{c}-G^{*}\right)\left(G^{*} g_{1}+\lambda\left(\bar{k}_{c}-G^{*}\right)\right)-c_{1} G^{*}\left(\bar{k}_{c}-G^{*}\right) \hat{A}_{s}}{\bar{c}_{1} G^{*}\left(k_{c}-G^{*}\right)-c_{1} G^{*}\left(\bar{k}_{c}-G^{*}\right) \hat{A}_{s}}
$$

where $\hat{A}_{s}=\left(A_{s} / A_{\infty}\right) e^{j\left(\phi_{s}-\phi_{\infty}\right)}$ and $\lambda=-\frac{3}{2}$. Although our implementation of this procedure differs in detail from the one given by Kiracofe and Raman (compare our Suppl. Info with theirs), we have checked that the $K^{*}$ values resulting from both implementations are identical.

\subsubsection{Thermal noise spectroscopy}

When using thermal noise spectroscopy (TNS), the cantilever is not driven macroscopically but undergoes thermal vibrational fluctuations. As described 
in Ref. [16], the power spectral density (PSD) of these fluctuations,

$$
P(\omega)=P_{w}+\frac{P_{0}}{\left(1-\left(\omega / \omega_{0}\right)^{2}\right)^{2}+\omega^{2} /\left(Q \omega_{0}\right)^{2}}
$$

is measured for several tip-substrate distances. Here $P_{w}$ accounts for the instrumental (white) noise and $P_{0}$ is a scaling factor. From these PSD's the quality factor $Q$ and the resonance frequency $\omega_{0}$ are obtained by fitting Eq. (4.9) to the data. The interaction parameters $k_{\text {int }}$ and $\gamma_{\text {int }}$ are determined from the changes in $Q$ and $\omega_{0}$ :

$$
k_{\mathrm{int}}=k_{c}\left(\frac{\omega_{0}}{\omega_{\infty}}\right)^{2}-k_{c} \quad \text { and } \quad \gamma_{\mathrm{int}}=\frac{k_{c} \omega_{0}}{Q \omega_{\infty}^{2}}-\gamma_{c}
$$

In Fig. 4.3 an approach curve, i.e. deflection versus mean distance from the substrate, has been given. The inset shows three spectra, measured at different distances as indicated by the arrows with corresponding color in the main figure, together with the best fits of Eq. (4.9).

\subsection{Procedures and analysis}

As mentioned in the introduction, we investigate three different phenomena to test the force inversion procedures for both conservative and dissipative forces. We measure the interaction and damping forces between a silica tip and silica substrate in an electrolyte environment and on a slightly shorter range the hydration forces between a silica tip and an atomic smooth mica substrate. To compare the dissipative force with the macroscopic hydrodynamic Reynolds damping we use a colloidal silica probe while measuring the approach curves towards a silica surface. In all three cases we use both AM and TNS.

\subsubsection{Materials and procedures}

Materials All experiments are performed with an aqueous salt solution of $\mathrm{NaCl}$ with varying strength and $\mathrm{pH}$ (by adding $\mathrm{HCl}$ or $\mathrm{NaOH}$ ). For the DLVO and hydrodynamic dissipation measurements, we use a $10 \mathrm{mM}$ concentration of $\mathrm{NaCl}$, resulting in a Debye length of approximately $3 \mathrm{~nm}$. To increase the surface charge on the silica surfaces - and so the DLVO interactions between tip and substrate - we enhanced the deprotonation of the silanol groups by adding $\mathrm{NaOH}$ resulting in a $\mathrm{pH}$ of 9 . On the other hand, to suppress the 

is acoustic driving in liquid quantitatively reliable?

electrostatic interaction in the dissipation experiments, we reduce the $\mathrm{pH}$ to 5 by adding $\mathrm{HCl}$. For the hydration force measurements a freshly cleaved mica substrate and a $400 \mathrm{mM}$ solution are used. A high salt concentration has in this case two advantages. First, the hydration oscillations are more pronounced, which leads to better reproducibility [21]. Second, the electrostatic interactions are effectively screened and will not dominate the oscillating hydration forces because the Debye length is less than $0.5 \mathrm{~nm}$.

Procedures For the DLVO and hydration force measurements a Bruker Multimode AFM is used with a Nanoscope V controller, E scanner and a fluid cell made from glass. During the measurements, the fluid cell is sealed to minimize evaporation. For the measurements with a colloidal probe a Bruker Icon AFM is used because it is more convenient for handling different liquids. The sample preparation and experimental settings are similar for all three cases. Prior to the measurements, the silica substrate and cantilevers are both rinsed in isopropanol and ethanol before they are cleaned in a plasma chamber (Harrick Plasma) for several minutes. The DLVO measurements are done with initially sharp tips obtained from Mikromasch (NSC36/Cr-Au BS, nominal $R \sim 10 \mathrm{~nm}$ ). To avoid variations of the tip curvature during measurement, we flatten the cantilever tip after it is immersed in the liquid by imaging the substrate in contact mode with a constant load of about $50 \mathrm{nN}$ during approximately 10 minutes. Next, we calibrate the cantilever parameters $Q_{\infty}=4.10, \omega_{\infty} / 2 \pi=42.25 \mathrm{kHz}$ and $k_{c}=2.3 \mathrm{~N} / \mathrm{m}$ by measuring the thermal spectrum [27] at a tip-substrate separation of about $30 \mathrm{~nm}$. The actual AM measurements are done at a driving frequency, $\omega / 2 \pi=41.00 \mathrm{kHz}$, just below the initial resonance frequency and with an amplitude $A_{\infty}=0.21$ $\mathrm{nm}$. We first measure amplitude and phase response as a function of the piezo displacement down to distances where the tip is in hard contact with the substrate. The approach curves have been plotted in Fig. 4.2. They are used to determine $A_{s}$ and $\phi_{s}$. Additionally we capture 58 approach curves at an approach speed of $100 \mathrm{~nm} / \mathrm{sec}$ (amplitude, phase and static deflection versus piezo displacement) avoiding hard contact in order not to damage the tip, using a set-point for motion reversal at an amplitude $A=0.6 A_{\infty}$. The averaged $A(d)$ and $\phi(d)$ curves (where $d$ is the tip-substrate distance) have been plotted in Fig. 4.4(a). They are used for the force inversion calculations.

After the AM measurements thermal noise spectra are taken as a function of piezo displacement. To obtain a full force distance curve, the substrate approaches the tip at a constant speed of $5 \mathrm{~nm} / \mathrm{s}$ while the thermally fluctuating deflection of the cantilever is captured at a rate of $500 \mathrm{kHz}$ using a low-pass filter with a cut-off frequency of $200 \mathrm{kHz}$. This is roughly four times the can- 
tilever resonance frequency $\omega_{\infty}$ of about $50 \mathrm{kHz}$. The signal that drives the substrate up and down is simultaneously monitored. The moment to reverse the motion from approach to retraction is determined by the set-point of the cantilever load, which is $2.5 \mathrm{nN}$. Defining this point as $d=\delta$, where $\delta$ is a constant close to zero $\mathrm{nm}$, we correct during every approach-retraction cycle for piezo drift which is about $0.1 \mathrm{~nm} / \mathrm{s}$. To obtain sufficient accuracy we capture several approach curves, like in the AM measurements. The exact number depends on the specific experiment and ranges typically from 10 to 100.

The hydrodynamic dissipation measurements (AM and TNS) are performed using colloidal probe cantilevers (Team Nanotec LRCH/Cr-Au). The approach speed is set to $100 \mathrm{~nm} / \mathrm{s}$ in case of AM and reduced to $3 \mathrm{~nm} / \mathrm{s}$ when TNS is applied. For the hydration force measurements we use the same cantilevers as for the DLVO case, without blunting them before measurement, but the settings are slightly different. It is known that the conservative part of hydration forces is quasi-periodic at tip-substrate distances close to the diameter of a water molecule, i.e $0.3 \mathrm{~nm}$ [21]. To probe this periodicity properly with AM, the amplitude of the cantilever oscillation is set to $0.12 \mathrm{~nm}$. Because this reduces the signal to noise ratio considerably, longer integration times are required. So we reduce the approach speed in this case to $1 \mathrm{~nm} / \mathrm{s}$. For TNS the approach speed is set to $0.3 \mathrm{~nm} / \mathrm{s}$. We have summarized all the experimental settings in Table S2 of the Suppl. Info.

\subsubsection{Data analysis}

To analyze the AM data, we use both the conventional SHO model and the continuous beam approach including the liquid mediated excitation contribution. When we use the SHO model, we determine $\hat{A}$ from the measured $A(d) e^{j \phi(d)}$ and $A_{\infty} e^{j \phi_{\infty}}$ values and calculate $K^{*}(d)$ using Eq. (4.6) with $C_{\text {sho }}=1$ and $B_{f}^{*}=0$. Similarly, with the continuous beam approach, we first calculate $\hat{A}_{s}$ from the measured $A_{s} e^{j \phi_{s}}$ and $A_{\infty} e^{j \phi_{\infty}}$ values and apply Eq. (4.8) to calculate $B_{f}^{*}$. Then we determine $\hat{A}$ and calculate $K^{*}(d)$ again with Eq. (4.6) but now with $C_{\mathrm{cbm}}=1.57$, see Eq. (4.7). For the DLVO force measurements, we find $B_{f}^{*}=(0.577+1.157 \mathrm{j})$, indicating that excitation via the fluid is significant. In case of the hydration force measurements, performed with the same instrument, same cantilever and same liquid cell, we obtain $B_{f}^{*}=(1.382+0.014 \mathrm{j})$, which is of the same order of magnitude but has a different phase angle. This can be due to a slightly different mounting of the cantilever in the cell. For the hydrodynamic dissipation measurements, 

is acoustic driving in liquid quantitatively reliable?

performed with the Icon instrument, we get $B_{f}^{*}=(0.566-0.142 \mathrm{j})$. Because $C_{\mathrm{cbm}}\left|1+B_{f}^{*}\right| \sim 3$ we expect to find a clear discrepancy between the results obtained with the SHO model and the continuous beam approach. This will be further discussed in Section 4.4.

In the TNS analysis, the recorded displacement sequences during each approach are divided into intervals of $2^{15}$ points [16]. At the given approach rate, the substrate travels less than $0.4 \mathrm{~nm}$ during each interval. Performing hydration force measurements, it is even less, $\sim 0.02 \mathrm{~nm}$. Consequently, each interval can be associated with a single tip-substrate distance $d$ with a resolution of $0.2(0.01) \mathrm{nm}$. To correct the time sequences for the (also interaction dependent) average deflection of the cantilever, the best fitting straight line to the sequence is substracted from that sequence, before it is transformed into a PSD. Next Eq. (4.9) is fitted to the experimentally obtained PSD from which $\omega_{0}, Q$, and using Eq. (4.10) also $k_{\text {int }}$ and $\gamma_{\text {int }}$ are determined. In the fitting process, the frequency data below $0.15 \omega_{0}$ are excluded to avoid contributions from the $1 / f$ noise. Also the high frequency data (above $2 \omega_{0}$ ) are omitted because these mix up with contributions from the first overtone. Figure 4.4(b) shows the $\omega_{0}$ and $Q$ versus distance curves obtained from the DLVO force measurements. They are the average of nine consecutive approach curves.

To compare the results of the AM and TNS method with each other (and with theoretical calculations), the tip-substrate distance should be known accurately. This distance is defined by the piezo position relative to the substrate plus the cantilever deflection. To determine the piezo position relative to the substrate, we analyze the approach curve when the tip is in hard contact with the substrate (see Figs. S1 and S2 of the Suppl. Info). For deflections above $10 \mathrm{~nm}$ we fit a straight line to the measured deflection versus piezo position curve (static deflection curve). Extrapolating this line to deflection zero gives us the piezo position at which a non-deflected tip would just touch the substrate. At this point the actual tip-substrate distance is equal to the cantilever deflection (point $P$ in Fig. S1 of the Suppl. Info). Simultaneously with each AM and TNS approach curve we also measure the static deflection curve. As these static deflection curves should match with each other, we can determine the point $P$ also for cases where no hard contact was established, see the black and red curves in Fig. S1 of the suppl. info. So we can determine the tip-substrate distance $\delta$ of the turning point in the approach curves for all cases. For the DLVO force measurements the TNS set point of $2.5 \mathrm{nN}$ (i.e. a deflection of $1.1 \mathrm{~nm}$ ) corresponds to a tip-substrate distance at the turning point of $\delta \simeq 0.2 \mathrm{~nm}$ (the end point of the black noisy 

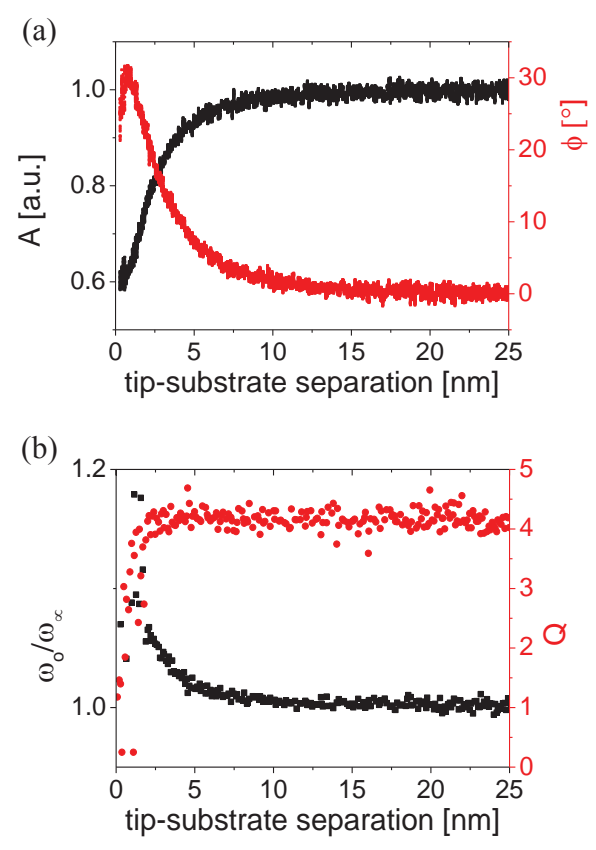

Figure 4.4: AM and TNS measurements in $10 \mathrm{mM} \mathrm{NaCl}$ on silica substrate. (a) Amplitude and phase, the data are averaged over 52 approaches. The amplitude is normalized with respect to $A_{\infty}$. (b) $\omega_{0} / \omega_{\infty}$ and $Q$ as obtained from the power spectra of the cantilever's thermal motion. The data are extracted from 9 approaches. Experimental parameters: Mikromasch NSC36/Cr-Au $\mathrm{BS}, k_{c}=2.30 \mathrm{~N} / \mathrm{m}, \gamma_{c}=2.06 \mu \mathrm{Ns} / \mathrm{m}, \omega_{\infty} / 2 \pi=42.25 \mathrm{kHz}, \omega / 2 \pi=41.00$ $\mathrm{kHz}, A_{\infty}=0.205 \mathrm{~nm}$. 

is acoustic driving in liquid quantitatively reliable?

curve in Fig. S1 of the Suppl. Info). The amplitude reduction set point of $A / A_{\infty}=60 \%$ in $\mathrm{AM}$ corresponds to $\delta \simeq 0.7 \mathrm{~nm}$ (the end point of the red noisy curve in Fig. S1). For the hydrodynamic dissipation measurements we used a TNS set point of $20 \mathrm{nN}$, corresponding to $\delta \simeq 0.5 \mathrm{~nm}$, while for AM we used an amplitude reduction set point of $A / A_{\infty}=25 \%$. This corresponds to $\delta \simeq 3.7 \mathrm{~nm}$. Measuring the hydration forces, we use another criterion. The profile of $k_{\text {int }}(d)$ against piezo displacement shows clear maxima and minima. These are used to align both the $k_{\text {int }}(d)$ and $\gamma_{\text {int }}(d)$ curves such that the average of the two largest local minima in $k_{\text {int }}(d)$ of all curves coincides. In this way we not only optimize the accuracy of the tip-substrate distance, but also prevent smearing out of the $k_{\text {int }}(d)$ and $\gamma_{\text {int }}(d)$ curves when averaging over a large number of curves.

\subsection{Results}

In this section, we present the results of the three experiments and, when appropriate, compare them with theoretical predictions. The results from the AM analysis using the simple harmonic oscillator model are referred to as SHO, those of the continuous beam approach as CBM.

\subsubsection{DLVO and squeeze-out force with sharp tips}

Figure 4.4 shows the primary results from the DLVO force experiment. Both the $A(d), \phi(d)$ and the $\omega_{0}(d)$ curves show a characteristic transition near a tip-substrate distance of $5 \mathrm{~nm}$, reflecting the range of the electrostatic interactions which is given by the Debye length, i.e. $3 \mathrm{~nm}$ for a $10 \mathrm{mM}$ salt solution. The resulting force gradient (interaction stiffness) and dissipation have been shown in Fig 4.5. We observe a good agreement between the interaction stiffness results obtained with TNS, CBM and SHO. All data points agree with each other within $0.03 \mathrm{~N} / \mathrm{m}$. As an additional check, we also determine the interaction stiffness from the static deflection versus distance curves, by taking the derivative of the static deflection curve with respect to distance, multiplied by the intrinsic stiffness of the cantilever $k_{c}$, the gray curve in Fig. 4.5. As can be observed from the figure, the obtained values match well with the interaction stiffness that was measured using AM or TNS. However, the interaction damping obtained from the SHO analysis deviates from the other results, and reveals even an unrealistic negative interaction damping coefficient. 


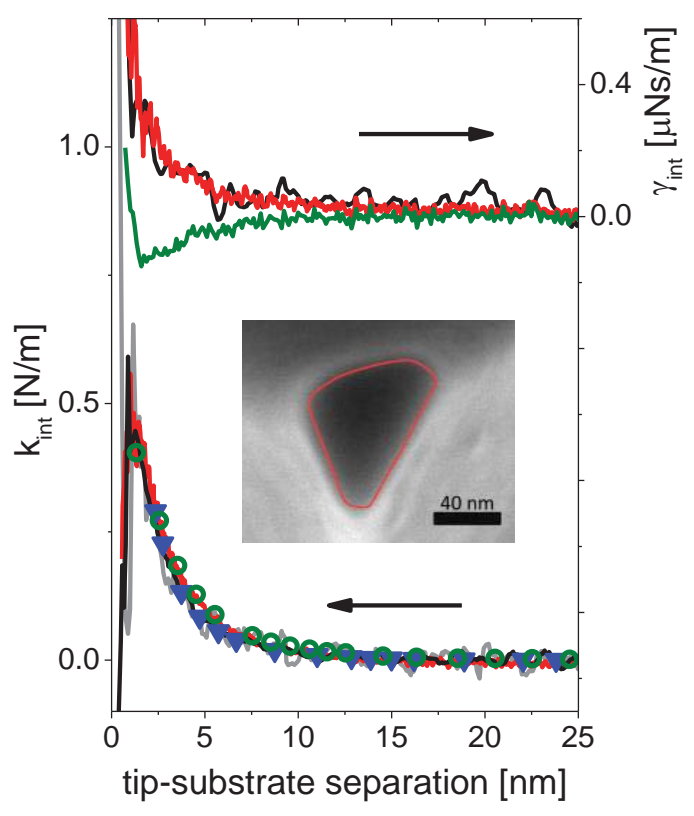

Figure 4.5: Interaction stiffness and damping coefficient versus distance as obtained from AM using the SHO model (green line and circles), using the CBM approach (red), from TNS (black) and from static deflection (gray). The blue triangles represent the calculated interaction stiffness based on the DLVO theory, Eq (4.11). The inset shows a SEM image of the contact area (within the red contour) of the tip after use. Experimental settings are the same as in Fig. 4.4. 

is acoustic driving in liquid quantitatively reliable?

DLVO calculation The conservative DLVO force $F$ between tip and substrate results from three contributions, i.e. the osmotic, electrostatic and van der Waals force:

$$
F=S\left\{k_{B} T \sum_{i} n_{i}^{\infty}\left(e^{-\frac{Z_{i} e \phi}{k_{B} T}}-1\right)-\frac{\varepsilon_{0} \varepsilon_{r}}{2}\left(\frac{\partial \phi}{\partial x}\right)^{2}-\frac{A_{H}}{6 \pi d^{3}}\right\}
$$

Here $S$ is the interaction area of the tip which was flattened as described in Section 4.3.1. The first term within the braces represents the osmotic contribution with $\phi$ the electric potential, $e$ the elementary charge and $k_{B} T$ the thermal energy. $Z_{i}$ is the valency and $n_{i}^{\infty}$ the bulk concentration of ions of species $i$. In our experiment four ionic species are present, as there are cations and anions from dissolved $\mathrm{NaCl}$ as well as hydroxide and hydrogen ions due to auto hydrolysis. The second term represents the electrostatic contribution with $\varepsilon_{0}$ the electric permittivity of vacuum and $\varepsilon_{r}$ the relative dielectric constant of water. In the third term, $A_{H}$ represents the Hamaker constant and $d$ the tip-substrate separation. Calculation of the osmotic and electrostatic contribution requires the solution of the electric potential between the tip and the substrate. This potential is governed by the Poisson-Boltzmann equation:

$$
\varepsilon_{0} \varepsilon_{r} \frac{\partial^{2} \phi}{\partial x^{2}}=-\sum_{i} e Z_{i} n_{i}^{\infty} e^{-\frac{Z_{i} e \phi}{k_{B} T}}
$$

Last equation is conventionally solved by assuming either constant charge or constant potential on tip and substrate. These assumptions are justified for large tip-substrate separations (where tip and substrate only weekly interact with each other), but usually fail in the regime of small tip-substrate distances. In this regime $\left(\kappa d<10\right.$, where $\kappa=\left(e^{2} \sum Z_{i}^{2} n_{i}^{\infty} / \varepsilon_{0} \varepsilon_{r} k_{B} T\right)^{1 / 2}$ is the reciprocal Debye length) the local charge density and potential vary with separation distance $d$ to compensate the confinement-induced modification of the surface chemistry. Hence, the boundary conditions for Eq. (4.12) are controlled by charge regulation $[19,28,29]$ :

$$
\sigma_{s}=\mathcal{F}_{\mathrm{cr}}\left(\phi_{s} ; n_{i}^{\infty}, \Gamma, p K_{H}, p K_{N a}, C_{S}\right)
$$

where $\sigma_{s}$ is the surface charge density of the substrates and $\phi_{s}$ the surface potential. Moreover, $\Gamma=\left\{\mathrm{S}^{-}\right\}+\{\mathrm{SH}\}+\{\mathrm{SNa}\}$ is the total site density of surface groups, $p K_{H}$ the equilibrium constant for protonation-deprotonation of the Silica tip and substrate, $\sim S H \rightleftharpoons \sim S^{-}+H^{+}, p K_{N a}$ the equilibrium constant for cation adsorption-desorption, $\sim S N a \rightleftharpoons \sim S^{-}+N a^{+}$and $C_{S}$ the capacity of the Stern layer with thickness $d_{S}$. The exact dependence of $\sigma_{s}$ on $\phi_{s}$ has 
been given in the Suppl. Info, see also [19]. As $\left[\mathrm{Na}^{+}\right],\left[\mathrm{Cl}^{-}\right],\left[\mathrm{H}^{+}\right],\left[\mathrm{OH}^{-}\right]$ are known and $\Gamma, p K_{H}, p K_{N a}, C_{S}$ can be obtained from literature, only the interaction area $S$ is unknown. Therefore we use $S$ as fitting parameter, when we fit the calculated $-\partial_{d} F(d)$ curve to the experimental $k_{\text {int }}$ values obtained with TNS. Data at separations $d<2.5 \mathrm{~nm}$ are omitted from this procedure, because the DLVO theory, as it is continuous in nature, is inadequate to describe the interactions at these small distances [17]. An example of such interactions is the hydration force we study in Fig. 4.7 (see Section 4.4.3).

The calculated interaction stiffness $k_{\text {int }}=-\partial_{d} F(d)$ has been plotted in Fig. 4.5, too. It matches very convincingly with both the AM and TNS results. The fitted tip area $S$ is $1900 \mathrm{~nm}^{2}$. This is rather close to the area obtained from the SEM image of the tip after use, which is approximately $2000 \pm 100 \mathrm{~nm}^{2}$, see the inset of Fig. 4.5. This agreement with commonly accepted theory demonstrates the validity of both approaches at least for the conservative part of the tip-substrate interaction.

\subsubsection{Hydrodynamic dissipation with a colloidal probe}

In Fig. 4.6 the results from the colloidal probe force spectroscopy have been shown. In this case the agreement between the TNS and AM results is still reasonable but less convincing than in the previous case. For the interaction damping $\gamma_{\text {int }}$ we observe a fair agreement between the CBM and TNS results although they deviate slightly for $d<6 \mathrm{~nm}$. In this range the $\mathrm{SHO}$ results for the damping coefficient are systematically smaller than those from the other two approaches. The TNS results for the interaction stiffness $k_{\text {int }}$ agree nicely with the stiffness calculated from the static deflection curve, which is captured simultaneously with the AM amplitude and phase curves. However, the AM results deviate significantly from both. The discrepancy starts at about $d=8 \mathrm{~nm}$, and it increases to about $50 \%$ at $4 \mathrm{~nm}$. Note that also in this case $\mathrm{SHO}$ and CBM give the same results for the conservative part of the interaction. The discrepancy between the TNS and CBM analysis is probably caused by the accuracy with which the $B_{f}^{*}$ coefficient can be determined (see Section 4.2.1). To determine $B_{f}^{*}$ one needs to measure $A_{s} / A_{\infty}$ (see Fig. S2 of the Suppl. Info). However, in this case $A_{s} / A_{\infty}$ does not show a plateau for large cantilever deflections, but increases with decreasing piezo distance with a slope of $-1 \% / \mathrm{nm}$. So the estimate for $A_{s} / A_{\infty}$ is inaccurate. Specially the obtained values for $\gamma_{\text {int }}$ are sensitive to variations in $A_{s}$ (see Fig. S3 of the Suppl. Info).

For a colloidal probe near a flat substrate, the expected hydrodynamic 


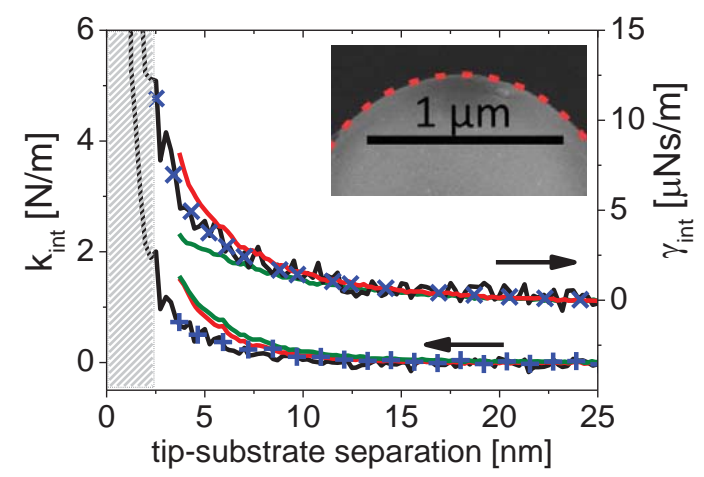

Figure 4.6: Interaction stiffness and damping versus distance obtained with a colloidal probe cantilever in a $10 \mathrm{mM} \mathrm{NaCl}$ solution on silica. The AM results (CBM: red, SHO: green) are the average of 10 approach curves, TNS results (black) of 3 approach curves. The interaction stiffness derived from static deflection is given by the blue + symbols. The blue $\mathbf{x}$ symbols represent the calculated damping coefficient. The inset shows a SEM of the tip after use. The dashed red line indicates the curvature of the tip apex, $R=800 \mathrm{~nm}$. Experimental parameters: Team Nanotec LRCH $/ \mathrm{Cr}-\mathrm{Au} \mathrm{BS}, k_{c}=4.13 \mathrm{~N} / \mathrm{m}$, $\gamma_{c}=5.52 \mu \mathrm{Ns} / \mathrm{m}, \omega_{\infty} / 2 \pi=35.60 \mathrm{kHz}, Q_{\infty}=3.50, \omega / 2 \pi=34.00 \mathrm{kHz}$, $A_{\infty}=0.38 \mathrm{~nm}$. 
Reynolds damping for a Newtonian liquid with viscosity $\eta$ under no-slip conditions is given by:

$$
\gamma_{\text {int }}(d)=6 \pi \frac{\eta R_{\text {tip }}^{2}}{d-\Delta}-\gamma_{\text {offset }}
$$

Since for practical reasons $\gamma_{\text {int }}$ is defined to be zero for $d \geq d_{c}=25 \mathrm{~nm}$, $\gamma_{\text {offset }}=6 \pi \eta R_{\text {tip }}^{2} /\left(d_{c}-\Delta\right) . \Delta$ is an offset to compensate for the uncertainty in the tip-substrate separation. We fit Eq. (4.14) to the measured interaction damping as obtained from the TNS analysis for separations larger than $2.4 \mathrm{~nm}$, using $\eta R_{\text {tip }}^{2}$ and $\Delta$ as fitting parameters. We obtain for $\eta R_{\text {tip }}^{2}=$ $(1.06 \pm 0.05) \times 10^{-15} \mathrm{Ns}$. The radius obtained from the SEM image of the tip after use is $0.8 \pm 0.1 \mu \mathrm{m}$, resulting in a viscosity $\eta=1.7 \pm 0.4 \mathrm{mPa}$, which is almost twice the viscosity of pure water at $T=25^{\circ} \mathrm{C}, \eta=0.89 \mathrm{mPa}$. As both the tip and the substrate are negatively charged this discrepancy can be caused by the electroviscous effect, which indeed under certain circumstances can enhance the viscosity by at least $50 \%$ [30]. In a future publication we will address this issue in more detail. For the offset in the tip-sample distance we find $\Delta=0.8 \mathrm{~nm}$. Last value suggests that there is a stagnant hydration layer present at both the tip and the substrate with a thickness of about $0.3 \mathrm{~nm}$, as discussed by [31]. The remaining $0.2 \mathrm{~nm}$ is of the order of the accuracy with which the tip-substrate distance can be determined.

We have masked the results in Fig. 4.6 for distances below $2.5 \mathrm{~nm}$ because in this range the TNS data become inaccurate due to the low quality factor. The agreement between the measured and the theoretical Reynolds damping demonstrates that the TNS approach is not only valid for the conservative part but also for the dissipative part of the tip-substrate interaction. The $\mathrm{CBM}$ approach is in this case less accurate, leading to relative errors up to $15 \%$.

\subsubsection{Oscillatory hydration force}

As a final test case, we study oscillatory hydration forces between a silica tip and mica in aqueous $0.4 \mathrm{M} \mathrm{NaCl}$ solution. Oscillatory hydration forces are less ideal as a validation case in the sense that there is no generally accepted theory to compare to. At the same time, this makes them a particularly interesting test case because of challenging open scientific questions. While the conservative part of the force oscillations has been well established since the early measurements with the Surface Forces Apparatus (SFA) in the 1980s $[17,31]$, the behavior of the dissipation in confined liquids has been a 


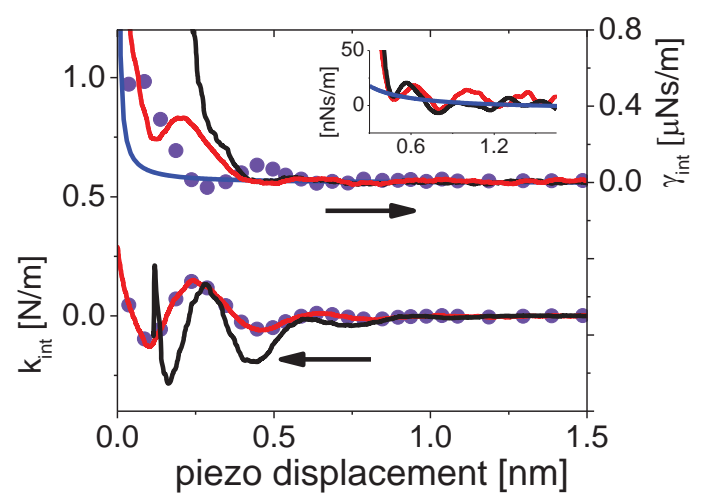

Figure 4.7: Interaction stiffness and damping coefficient versus distance of an AFM tip in $400 \mathrm{mM} \mathrm{NaCl}$ on mica. The AM results (CBM: red curves, SHO: blue dots) are averaged over 81 approaches, TNS results (black) over 30 approaches. The blue line represents the Reynolds damping for a tip radius of $20 \mathrm{~nm}$. The cantilever calibration as described in Fig. 4.4 is carried out before recording the amplitude-phase-distance curves. The inset shows the oscillations in the damping, visible in both TNS and CBM. Experimental parameters: Mikromasch NSC36/Cr-Au BS, $k_{c}=1.00 \mathrm{~N} / \mathrm{m}, \gamma_{c}=1.66 \mu \mathrm{Ns} / \mathrm{m}$, $\omega_{\infty} / 2 \pi=29.11 \mathrm{kHz}, Q_{\infty}=3.25, \omega / 2 \pi=29.40 \mathrm{kHz}, A_{\infty}=0.12 \mathrm{~nm}$.

subject of vivid debates ever since $[6,8,15,20,25,32]$. With the advent of dynamic AFM measurements that enable stabilization of arbitrary tip-sample distances (no mechanical jump-to-contact instabilities) the focus of the attention has shifted towards the specific question whether the strong modulation of the conservative forces and the density of the fluids is reflected in a non-monotonic dependence of the dissipation on the tip-sample distance [32]. Figure 4.7 shows the stiffness and the dissipative part of the hydration forces as a function of tip-sample distance. All three methods, the TNS and the SHO and CBM analysis of the actively driven AM-AFM measurements display oscillations of the stiffness at separations below $1 \mathrm{~nm}$. The distance between adjacent peaks is $0.3 \pm 0.03 \mathrm{~nm}$, which is typical for the thickness of a layer of water as reported before. The SHO and CBM analysis of the driven measurements overlap perfectly, in line with the observations for the conservative DLVO forces in Figs. 4.5 and 4.6. Both SHO and CBM measurements display a somewhat smaller amplitude though, than the TNS measurement. This is not surprising because we linearize the tip-sample interaction in the 
course of our data analysis (see section 4.2.1). In earlier measurements and numerical calculations for the reference case of Octamethylcyclotetrasiloxane (OMCTS), we quantified how the measured strength of oscillatory solvation forces decreases with increasing cantilever oscillation amplitude [11]. It was demonstrated there that the actual strength is only recovered for cantilever amplitudes below approx. 10-15\% of the diameter of the molecule in question. For water, would require a drive amplitude below $50 \mathrm{pm}$, i.e. less than half the value $0.12 \mathrm{~nm}$ used in our present measurements. Extrapolating from the results in ref. [11], which assume that the solvation forces can be described as an exponentially decaying cosine function, we find that the intrinsic amplitude of the oscillatory forces should be approximately 2.5 times stronger than displayed in Fig. 4.7. The result is rather close in amplitude $(\approx 30 \%$ larger) compared to the TNS. Yet, the stiffness obtained by TNS displays a slight negative background qualitatively consistent with the static deflection, which is absent in the AM-AFM data. Upon integration, this would lead to a net attractive force (see Suppl. Info). At this stage, we do not have an explanation for this deviation. (It is tempting to apply existing force inversion formulas for AM-AFM for arbitrary oscillation amplitudes [33]. Applying this method indeed increases the amplitude of the oscillations in the recovered solvation forces. Yet, we refrain from showing these data because this approach, which is based on the Sader-Jarvis formalism, has been reported to fail for dissipative forces, in particular in liquid environment and in the presence of steep gradients of the conservative force [33,34].) For the dissipative forces, the SHO analysis strongly deviates from the result obtained by both TNS and CBM, similar to the case of the viscous dissipation with slightly blunted tips reported in Fig. 4.5. TNS becomes unreliable below approx. $0.25 \mathrm{~nm}$, when the cantilever motion becomes overdamped. For larger separations, however, TNS and CBM agree and both consistently reveal a non-monotonic behavior of $\gamma_{\text {int }}$ with local minima of the dissipation around $0.45 \mathrm{~nm}$ and $0.8 \mathrm{~nm}$ and consistent a local maximum around $0.6 \mathrm{~nm}$ (see inset Fig. 4.7). Figure 4.7 also shows that the expected viscous dissipation based on the bulk viscosity of water for an assumed tip radius of $20 \mathrm{~nm}$ is consistent with the damping coefficient extracted from the experiments for tip-sample distances beyond $\approx 0.6 \mathrm{~nm}$. It is worthwhile to note that the amount of energy dissipated in this flow is rather small. For the present system, we can provide an upper limit of the power dissipated between tip and sample by $P \approx \gamma_{\text {int }}(\omega A)^{2} \approx 4 \times 10^{-18} \mathrm{~W}$. For each oscillation cycle with a period of $\tau=30 \mu \mathrm{s}$, this corresponds to an amount of dissipated energy of $1 \times 10^{-22} \mathrm{~J} \approx 0.03 k_{B} T$. This very small number, which is roughly consistent with the reports by Labuda et al. [25], corresponds to a fraction of the latent 

is acoustic driving in liquid quantitatively reliable?

\begin{tabular}{|c|c|c|c|c|c|c|}
\hline & \multicolumn{4}{|c|}{ DLVO and Hydr. squeeze-out } & \multicolumn{2}{|c|}{$\begin{array}{c}\text { Osc. Hydration } \\
R_{\text {tip }}=20 \mathrm{~nm}\end{array}$} \\
\hline$k_{c}[\mathrm{~N} / \mathrm{m}]$ & \multicolumn{2}{|c|}{2.30} & \multicolumn{2}{|c|}{4.13} & \multicolumn{2}{|c|}{1.00} \\
\hline$k_{\text {int }}^{\max }[\mathrm{N} / \mathrm{m}]{ }^{\dagger}$ & \multicolumn{2}{|c|}{0.5} & \multicolumn{2}{|c|}{2.0} & \multicolumn{2}{|c|}{0.5} \\
\hline$\gamma_{c}[\mu \mathrm{Ns} / \mathrm{m}]$ & \multicolumn{2}{|c|}{2.06} & \multicolumn{2}{|c|}{5.52} & \multicolumn{2}{|c|}{1.66} \\
\hline$\left.\gamma_{\text {int }}^{\max }[\mu \mathrm{Ns} / \mathrm{m}]\right]^{\dagger}$ & \multicolumn{2}{|c|}{0.4} & \multicolumn{2}{|c|}{10.0} & \multicolumn{2}{|c|}{0.5} \\
\hline & $\Delta k_{\text {int }}$ & $\Delta \gamma_{\text {int }}$ & $\Delta k_{\text {int }}$ & $\Delta \gamma_{\text {int }}$ & $\Delta k_{\text {int }}$ & $\Delta \gamma_{\text {int }}$ \\
\hline & $\overline{k_{\text {int }}^{\max }}$ & $\overline{\gamma_{\text {int }}^{\max }}$ & $\overline{k_{\text {int }}^{\max }}$ & $\overline{\gamma_{\text {int }}^{\max }}$ & $\overline{k_{\text {int }}^{\max }}$ & $\overline{\gamma_{\text {int }}^{\max }}$ \\
\hline Static Defl. & \pm 0.05 & - & \pm 0.02 & - & $\pm 0.10^{\sharp}$ & - \\
\hline Theory $\ddagger$ & \pm 0.05 & - & - & \pm 0.01 & - & - \\
\hline $\mathrm{CBM}$ & \pm 0.05 & \pm 0.15 & +0.40 & +0.08 & $\pm 0.30^{\sharp}$ & \pm 0.13 \\
\hline $\mathrm{SHO}$ & \pm 0.05 & -1.25 & +0.40 & -0.10 & $\pm 0.30^{\sharp}$ & \pm 1.00 \\
\hline
\end{tabular}

Table 4.1: Relative deviations with respect to TNS for the three different types of interactions. Indicated are the largest deviations, scaled on $k_{c}$ and $\gamma_{c}$, respectively. $(+)$ the measured values are systematically larger than the corresponding TNS values; $(-)$ they are systematically smaller; $( \pm)$ the values scatter around the corresponding TNS values. ( $\dagger$ ) $k_{\text {int }}^{\max }$ and $\gamma_{\text {int }}^{\max }$ are an estimate of the maximum values in the considered distance range. ( $\ddagger)$ These values were obtained after fitting the theoretical model to the TNS measurements, see text. $(\sharp)$ Values are based on the amplitude of the spatial oscillation.

heat of melting per water molecule in the bulk $\Delta H_{b}=6 \mathrm{~kJ} /$ mole $\approx 10^{-20} \mathrm{~J}$. This is consistent with the idea that the confined water layers remain highly mobile and that the AFM measurement does not drive the system very far out of equilibrium.

\subsection{Discussion}

To summarize our findings we collected all results in Table 4.1. As $k_{\text {int }}$ and $\gamma_{\text {int }}$ strongly vary with tip-substrate distance and can become very small for larger distances, we scale the deviations $\Delta k_{\mathrm{int}}$ and $\Delta \gamma_{\mathrm{int}}$ on the maxima $k_{\mathrm{int}}^{\max }$ and $\gamma_{\text {int }}^{\max }$, respectively. We observe that the static deflection measurements are in good agreement with the corresponding TNS results. Also the the- 
oretical calculations are in good agreement with the TNS data; the values obtained for the fit parameters are in both cases realistic, the fitted tip area matches nicely with the value obtained from the SEM pictures, while also the viscosity enhancement due to electroviscous dissipation seems realistic.

Moreover, the dissipative forces obtained with SHO deviate significantly from those obtained from CBM or TNS. But in all experiments carried out in this study, the conservative forces, which were extracted using the SHO model, agree quantitatively with the values extracted using the continuous beam model. While the latter is definitely superior in describing the details of the actual motion of the cantilever, this observation implies that the effect of the fluid-mediated excitation is implicitly corrected for by the calibration procedure described in $[11,35]$, even if the actual base motion of the cantilever is not properly described as noted by Kiracofe and Raman [14]. This observation is quite surprising and we do not have a simple physical explanation for it. It is also at variance with the report in [14], but it is consistent with our earlier findings [11]; the observed agreement is clear, both with the TNS measurements and with the static deflection curves as well as with the theoretical predictions. From a practical perspective, using the simpler SHO model calibration procedures of refs. $[11,35]$ has the additional advantage that it does not require hard mechanical contact between tip and sample. While this crucial step of the calibration procedure in the continuous beam model can obviously be carried out at the end of each experiment, it still implies that the tip can be damaged. Ex situ characterization of the AFM tip by high resolution scanning electron microscopy then no longer provides the actual geometry during the measurements. At last we conclude from Table 4.1 that all data obtained with the CBM approach are within $40 \%$ accuracy in agreement with the corresponding TNS data.

While the main focus of our experiments was to provide an experimental validation of the AM-AFM method proposed in ref. [14] using DLVO and hydrodynamic squeeze-out forces, it is also worthwhile to comment on the non-monotonic variations of the damping in the presence of oscillatory solvation forces in Fig. 4.7, which is at variance with the conclusions in ref. [25]. Taking a step back, it is clear that early AM-AFM measurements [32] also suffered from similar cross-talk between conservative and dissipative force channels, as pointed out by Kaggwa et al. [20] and analyzed in detail by de Beer et al. [11]. On the other hand, the early FM-AFM measurements with OMCTS [20], neglected that a very similar coupling occurs in FM-AFM, too, as demonstrated recently by Labuda et al. [25]. Moreover, the measurements in [20] suffered from excessive drive amplitudes compared to the size of the molecules and the use of force inversion formulas that have been reported 

is acoustic driving in liquid quantitatively reliable?

to be questionable for the reconstruction of dissipative forces in the presence of strongly varying conservative forces $[33,34]$ and to the best of our knowledge have not been validated for these conditions. As a consequence, Kaggwa et al. concluded that even for OMCTS, the dissipation increases monotonically with decreasing distance, which is in contrast to our more recent TNS measurements with OMCTS [16] and AM-AFM [11] that both do show non-monotonic dissipation. For water, the situation is similar. The present AM-AFM and TNS measurements as well as the off-resonance AMAFM measurements by Khan et al. [36] show non-monotonic dissipation. The FM-AFM measurements by Labuda et al. [25] do display oscillations in the raw dissipation signal of their FM-AFM measurements, too, but they attribute them exclusively to cross-talk between conservative and dissipative interactions. At the same time, the absolute order of magnitude of the dissipated energy is the same. At this stage, we do not see any convincing explanation for the differences between our data and ref. [25]. It should be noted, though, that beyond the largely technical debates on measurement and analysis procedures, evidence is growing that the question whether the damping of tip-sample interaction forces in AFM measurements of confined liquids is per se oscillatory or not may not be entirely well-posed. To explain the atomic resolution contrast on one hand and the small but measurable increase in dissipation at distances of $0.2-1 \mathrm{~nm}$, Labuda et al. [25] invoke a specific tip geometry consisting of an atomically small cluster that is responsible for the high resolution and a substantially blunter part of the tip with a rather large tip radius $(50 \mathrm{~nm})$. In fact, the tip was intentionally crashed into the surface prior to the measurement in order to create the small cluster that was need for high resolution imaging. Very plausibly they argue along with earlier theoretical suggestions $[37,38]$ that the few water molecules involved in mediating the interaction between the cluster and the surface cannot dissipate enough energy to generate a measurable signal. The larger blunter part is therefore invoke to explain the measured increase in dissipation. Whether such an increase in dissipation is oscillatory or not depends on the resulting local tip geometry, as has been confirmed by molecular dynamics simulations with simple Lennard Jones systems [39]. Only blunt but smooth tips - in the extreme case the two surfaces of an SFA - probe the intrinsic properties of a confined liquid. Sharp tips, however, probe an intrinsically three-dimensional system. From this perspective, a conclusion of the deviations between the present measurements and ref. [25] could be that the uncertainties arising from the unknown details of the tip geometry are actually more important than the differences between measurement techniques of carefully executed TNS, AM-AFM, and FM-AFM measurements. 
In this study we did not consider frequency modulation AFM force spectroscopy (FM-AFM) with piezo excitation under liquid. In AM-AFM, the effect of the spurious peaks in the transfer function, which are caused by cell geometry and the driving piezo as demonstrated by Kiracofe and Raman [14] can be reduced to a single complex number. This number depends on the excitation frequency. Yet, as long as the latter is kept constant and as long as it is guaranteed that the spurious peaks do no change during the measurements (e.g. due to evaporation of fluid), this contribution is canceled out by the calibration measurement of the free amplitude. For FM-AFM, the excitation frequency varies by definition as the forces between tip and sample change. Hence, the frequency-dependent correction factor varies with tip-sample distance. As a consequence one has to determine the transfer function of the driving piezo itself. This is possible, as demonstrated by Labuda et al. [10]. Yet, the resulting force inversion procedure is rather complex and we are not aware of any validation study that quantifies the reliability of FM-AFM as we do in this case for AM-AFM.

\subsection{Conclusion}

From our experimental comparison of amplitude modulation (AM) with acoustic excitation in liquid - with and without fluid mediated driving - with thermal noise spectroscopy (TNS), we conclude that conservative forces can be measured consistently within at least $40 \%$ accuracy for all three approaches. If the drive amplitude is sufficiently small to justify linearization of the tipsample interaction, one can restrict oneself to the more straightforward small amplitude analysis, in other cases the approach of de Beer et al. [11] or Katan et al. [33] should be used. Weak interaction stiffnesses that decay or increase monotonically with tip-substrate distance, like that of DLVO forces, can be measured using sharp tips (interaction area $\sim 10^{-3} \mu \mathrm{m}^{2}$ ) even within $5 \%$ accuracy with all investigated approaches. Accurate measurement of dissipative forces (within $10 \%$ of the predictions of macroscopic fluid dynamics) requires thermal noise spectroscopy or the use of continuous beam theory, including the fluid mediated driving contribution. Moreover, the AM approach is faster than TNS, because TNS demands a much larger amount of data points to obtain reliable statistics. Hence, we have established that both TNS and AM-AFM provide a reliable method for force spectroscopy under liquid. Conservative forces can be determined by AM-AFM using the more straightforward and widely used simple harmonic oscillator approach with the same accuracy as when the continuous beam approach with fluid 

is acoustic driving in liquid quantitatively reliable?

mediated driving is used. For dissipative forces one can use TNS or AMAFM provided that the fluid mediated driving of the cantilever is taken into account.

\section{Acknowledgement}

We thank Dr. Kiracofe for sharing his matlab code with us. This work has been supported by the Foundation for Fundamental Research of Matter (FOM), which is financially supported by the Netherlands Organization for Scientific Research (NWO).

\section{A Appendix}

\section{A.1 Dynamics of a cantilever driven by a piezo under liquid}

Here we follow the approach of Kiracofe and Raman [1] and Xu and Raman [2] to analyze the behavior of an acoustically driven cantilever under liquid. The partial differential equation describing the time dependent shape of a vibrating cantilever under liquid reads:

$$
E I \partial_{x}^{4} z(x, t)+\rho A \partial_{t}^{2} z(x, t)=f_{h}(x, t)
$$

where

$$
f_{h}(x, t)=\int_{0}^{\infty}-\Gamma\left(t^{\prime}\right)\left(\ddot{z}\left(x, t-t^{\prime}\right)-\ddot{z}_{f}\left(x, t-t^{\prime}\right)\right) d t^{\prime}
$$

is the hydrodynamic force per unit length on the cantilever at position $x$. The fluid velocity, caused by the piezo disturbance at that position, is indicated with $v_{f}(x, t)$. This disturbance is assumed to depend linearly on the piezo displacement at the base of the cantilever:

$$
v_{f}(x, t)=\int_{0}^{\infty}-B_{f}\left(x, t^{\prime}\right) \dot{z}_{b}\left(t-t^{\prime}\right) d t^{\prime}
$$

where $B_{f}$ describes the coupling. Translating these three equations to the frequency domain yields:

$$
E I \partial_{x}^{4} \tilde{z}(x, \omega)-\omega^{2} \rho A \tilde{z}(x, \omega)=\tilde{f}_{h}(x, \omega)
$$

with

$$
\tilde{f}_{h}(x, \omega)=\omega^{2} \Gamma^{*}(\omega)\left\{\tilde{z}(x, \omega)-\tilde{z}_{f}(x, \omega)\right\}
$$


and

$$
\tilde{z}_{f}(x, \omega)=-B_{f}^{*}(x, \omega) \tilde{z}_{b}(\omega)
$$

Note that $B_{f}^{*}(x, \omega)$ relates the fluid velocity along the cantilever at position $x$ with the displacement of the driving piezo. This dependence on $x$ has been neglected. Moreover, $B_{f}^{*}(\omega)$ corresponds with $-A_{\text {fluid }}(\omega)$ as defined in [1]. Combining last three equations we get:

$$
E I \partial_{x}^{4} \tilde{z}(x, \omega)-\omega^{2} \rho A \tilde{z}(x, \omega)=\omega^{2} \Gamma^{*}(\omega)\left(\tilde{z}(x, \omega)+B_{f}^{*}(\omega) \tilde{z}_{b}(\omega)\right)
$$

To solve this equation they assume that the cantilever shape can be written as a linear combination of the shape profiles belonging to the different modes in free vibration:

$$
\begin{aligned}
\tilde{z}(x, \omega) & =\tilde{w}(x, \omega)+\tilde{z}_{b}(\omega) \\
\tilde{w}(x, \omega) & =\sum_{n} \tilde{u}_{n}(\omega) \psi_{n}(x)
\end{aligned}
$$

where $\psi(x)$ is normalized such that $L \psi^{\prime}(L)=1$. This is different from Kiracofe et al. but makes more sense because the measured signal will be proportional to $L \tilde{w}^{\prime}(L)=\sum \tilde{u}_{n} L \psi_{n}^{\prime}(L)$ in stead of $\tilde{w}(L)=\sum \tilde{u}_{n} \psi_{n}(L)$. Moreover, $\psi_{n}^{\prime \prime \prime \prime}(x)=q_{n}^{4} \psi_{n}(x)$ with

$$
\left(q_{n} L\right)^{4}=\omega_{n}^{2} \frac{L\left(\rho A+\Gamma_{n}^{\prime}\right)}{E I / L^{3}}
$$

To fulfill the boundary conditions for free vibration:

$$
\psi(0)=0, \quad \psi^{\prime}(0)=0, \quad \psi^{\prime \prime}(L)=0 \quad \text { and } \quad \psi^{\prime \prime \prime}(L)=0
$$

one obtains for $q_{n} L$ the condition:

$$
\cos q_{n} L \cosh q_{n} L+1=0
$$

The shape mode functions are given by:

$$
\psi_{n}(x)=S_{n}\left(\cosh q_{n} x-\cos q_{n} x\right)-C_{n}\left(\sinh q_{n} x-\sin q_{n} x\right)
$$

where

$$
S_{n}=\frac{\sinh q_{n} L+\sin q_{n} L}{2 q_{n} L \sinh q_{n} L \sin q_{n} L}, \quad C_{n}=\frac{\cosh q_{n} L+\cos q_{n} L}{2 q_{n} L \sinh q_{n} L \sin q_{n} L}
$$


The values for $q_{n} L$ for the first 4 modes have been listed in Table S1, together with the corresponding resonance frequencies scaled on the reference frequency:

$$
\omega_{\mathrm{ref}}=\sqrt{\frac{E I / L^{3}}{L\left(\rho A+\Gamma_{n}^{\prime}\right)}}
$$

For higher modes the $q_{n} L$ values are just given by $q_{n} L=\left(n-\frac{1}{2}\right) \pi$.

\begin{tabular}{l|l|l}
$\mathrm{n}$ & $q_{n} L / \pi$ & $\nu_{n}$ \\
\hline 1 & 0.596863 & 003.5160 \\
2 & 1.494176 & 022.0344 \\
3 & 2.500247 & 061.6972 \\
4 & 3.499989 & 120.9019
\end{tabular}

Table S1: The numerical values for $q_{n}$ and $\nu_{n}=\omega_{n} / \omega_{\text {ref }}$ for the first 4 modes.

Substituting Eqs. (a5) and (a6) into Eq. (a4) we get:

$$
\sum_{n}\left(E I q_{n}^{4}-\omega^{2}\left(\rho A+\Gamma^{*}\right)\right) \tilde{u}_{n} \psi_{n}(x)=\omega^{2}\left(\rho A+\Gamma^{*}\left[1+B_{f}^{*}\right]\right) \tilde{z}_{b}
$$

Using orthogonality of the mode functions, Eq. (a11) is left and right multiplied with $\psi_{m}(x)$ and integrated over the length $L$ of the cantilever:

$$
\left(E I q_{m}^{4}-\omega^{2}\left(\rho A+\Gamma^{*}\right)\right) \tilde{u}_{m}\left\langle\psi_{m}^{2}\right\rangle=\omega^{2} \tilde{z}_{b}\left(\rho A+\Gamma^{*}\left[1+B_{f}^{*}\right]\right)\left\langle\psi_{m}\right\rangle
$$

where the average is defined by $\langle f\rangle=L^{-1} \int_{0}^{L} f(x) d x$. Defining $c_{m}=$ $\left\langle\psi_{m}\right\rangle /\left\langle\psi_{m}^{2}\right\rangle$ we arrive at:

$$
\frac{\tilde{u}_{m}}{\tilde{z}_{b}}=\frac{c_{m} \omega^{2} L\left(\rho A+\Gamma^{*}+\Gamma^{*} B_{f}^{*}\right)}{E I / L^{3}\left(q_{m} L\right)^{4}-\omega^{2} L\left(\rho A+\Gamma^{*}\right)}
$$

If we measure close to the first resonance peak of the cantilever, i.e. $\tilde{w}=$ $\sum_{n} \tilde{u}_{n} \psi_{n}(L) \simeq \tilde{u}_{1} \psi_{1}(L)$, so $L \tilde{w}^{\prime} \simeq \tilde{u}_{1} L \psi_{1}^{\prime}(L)=\tilde{u}_{1}$, we can rewrite Eq. (a13) as:

$$
\begin{aligned}
\frac{A_{\infty} e^{j \phi_{\infty}}}{A_{b} e^{j \phi_{b}}} & =c_{1} \frac{\omega^{2} m_{\mathrm{eff}}-j \omega \gamma_{c}+\omega^{2} m_{\mathrm{add}} B_{f}^{*}-j \omega \gamma_{c} B_{f}^{*}}{k_{c}-\omega^{2} m_{\mathrm{eff}}+j \omega \gamma_{c}} \\
& =c_{1} \frac{G^{*}\left(1+B_{f}^{*}\right)-\omega^{2} m_{c} B_{f}^{*}}{k_{c}-G^{*}}
\end{aligned}
$$


where $k_{c}=E I / L^{3}\left(q_{1} L\right)^{4}$ is the cantilever stiffness, $m_{c}=L \rho A$ its mass, $m_{\mathrm{add}}=L \Gamma^{\prime}$ the added mass, $m_{\mathrm{eff}}=m_{c}+m_{\mathrm{add}}$, and $\gamma_{c}=-\omega L \Gamma^{\prime \prime}$ the cantilever background damping. Moreover, $G^{*}=\omega^{2} m_{\mathrm{eff}}-j \omega \gamma_{c}$ ). Taking also in account that $\rho A \ll \Gamma^{\prime}$, Eq. (a14) reduces to:

$$
\frac{A_{\infty} e^{j \phi_{\infty}}}{A_{b} e^{j \phi_{b}}}=c_{1} \frac{G^{*}\left(1+B_{f}^{*}\right)}{k_{c}-G^{*}}
$$

Following the same line of thought we will derive an expression for $A_{s} e^{j \phi_{s}} / A_{b} e^{j \phi_{b}}$ in case the tip is in hard contact with the substrate. Now the cantilever deflection is described as:

$$
\tilde{w}(x, \omega)=\sum_{n} \tilde{u}_{n}(\omega) \chi_{n}(x)
$$

with boundary conditions $\chi(0)=\chi^{\prime}(0)=\chi^{\prime \prime}(L)=\chi(L)=0$. To obey these conditions the values of $\bar{q}_{n} L$ are in this case given by:

$$
\tan \bar{q}_{n} L=\tanh \bar{q}_{n} L
$$

or in good approximation:

$$
\bar{q}_{0} L=0, \quad \bar{q}_{n} L=\left(n+\frac{1}{4}\right) \pi \quad(n \geq 1)
$$

The shape functions $\chi_{n}(x)$ are identical to the $\psi_{n}(x)$, only the values for $q_{n} L$ and $\bar{q}_{n} L$ are different. The reason for this similar form of $\psi$ and $\chi$ is because both obey the boundary conditions $\chi(0)=\chi^{\prime}(0)=\chi^{\prime \prime}(L)=0$. They differ in the 4 th boundary condition resulting in different expressions $q_{n} L$ for each mode. To solve Eq. (a4):

$$
E I \partial_{x}^{4} \tilde{z}(x, \omega)-\omega^{2} \rho A \tilde{z}(x, \omega)=\omega^{2} \Gamma^{*}(\omega)\left\{\tilde{z}(x, \omega)+B_{f}^{*}(\omega) \tilde{z}_{b}(\omega)\right\}
$$

for the hard contact case, the cantilever shape can be written as a linear combination of the shape profiles belonging to the different modes in vibration in hard contact:

$$
\begin{gathered}
\tilde{z}(x, \omega)=\tilde{w}(x, \omega)+g(x) \tilde{z}_{b}(\omega) \\
\tilde{w}(x, \omega)=\sum_{n} \tilde{u}_{n}(\omega) \chi_{n}(x)
\end{gathered}
$$

where $g(x)$ is a function with $g(0)=1$ and $g^{\prime}(0)=g(L)=g^{\prime \prime}(L)=0$, just to match the boundary conditions for $\chi(x)$ with those for $\tilde{z}(x, \omega)$. The function 

is acoustic driving in liquid quantitatively reliable?

$g(x)=1-\frac{1}{2}(x / L)^{2}(3-x / L)$ fulfills these requirements. Moreover its 4 th derivative is zero. Substituting Eqs. (a19) and (a20) into Eq. (a4) we get:

$$
\sum_{n}\left(E I \bar{q}_{n}^{4}-\omega^{2}\left(\rho A+\Gamma^{*}\right)\right) \tilde{u}_{n} \chi_{n}(x)=\omega^{2}\left[\left(\rho A+\Gamma^{*}\right) g(x)+B_{f}^{*} \Gamma^{*}\right] \tilde{z}_{b}
$$

Using orthogonality of the mode functions once more, Eq.(a21) is left and right multiplied with $\chi_{m}(x)$ and integrated over the length $L$ of the cantilever:

$$
\left(E I \bar{q}_{m}^{4}-\omega^{2}\left(\rho A+\Gamma^{*}\right)\right) \tilde{u}_{m}\left\langle\chi_{m}^{2}\right\rangle=\omega^{2}\left[\left(\rho A+\Gamma^{*}\right)\left\langle g \chi_{m}\right\rangle+B_{f}^{*} \Gamma^{*}\left\langle\chi_{m}\right\rangle\right] \tilde{z}_{b}
$$

Defining $\bar{c}_{m}=\left\langle\chi_{m}\right\rangle /\left\langle\chi_{m}^{2}\right\rangle$ and $g_{m}=\left\langle g \chi_{m}\right\rangle /\left\langle\chi_{m}^{2}\right\rangle$, we arrive at:

$$
\frac{\tilde{u}_{m}}{\tilde{z}_{b}}=\frac{\omega^{2} L\left(g_{m}\left(\rho A+\Gamma^{*}\right)+\bar{c}_{m} \Gamma^{*} B_{f}^{*}\right)}{E I / L^{3}\left(\bar{q}_{m} L\right)^{4}-\omega^{2} L\left(\rho A+\Gamma^{*}\right)}
$$

The signal measured at the detector is given by:

$$
L \tilde{z}^{\prime}(L)=\sum_{n} \tilde{u}_{n} L \chi_{n}^{\prime}(L)+L g^{\prime}(L) \tilde{z}_{b}
$$

Because $L \chi_{n}^{\prime}(L)=1$ by normalization and $L g^{\prime}(L)=\lambda=-\frac{3}{2}$, we obtain:

$$
\frac{L \tilde{z}^{\prime}(L)}{\tilde{z}_{b}}=\frac{\sum_{n} \tilde{u}_{n}}{\tilde{z}_{b}}+\lambda
$$

As we are driving at a frequency below the first resonance frequency, again Kiracofe et al. assume that $\sum_{n} \tilde{u}_{n} \simeq \tilde{u}_{1}$ and the final result reads:

$$
\frac{L \tilde{z}^{\prime}(L)}{\tilde{z}_{b}}=\frac{\omega^{2} L\left(g_{1}\left(\rho A+\Gamma^{*}\right)+\bar{c}_{1} \Gamma^{*} B_{f}^{*}\right)}{E I / L^{3}\left(\bar{q}_{1} L\right)^{4}-\omega^{2} L\left(\rho A+\Gamma^{*}\right)}+\lambda
$$

or:

$$
\frac{A_{s} e^{j \phi_{s}}}{A_{b} e^{j \phi_{b}}}=\frac{G^{*}\left(g_{1}+\bar{c}_{1} B_{f}^{*}\right)}{\bar{k}_{c}-G^{*}}+\lambda
$$

where $\bar{k}_{c}=E I / L^{3}\left(\bar{q}_{1} L\right)^{4}$.

Finally, to obtain an expression for $\left(A e^{j \phi}\right) /\left(A_{b} e^{j \phi_{b}}\right)$ Kiracofe et al. state (see Eq. (39) of suppl. info in [1]):

$$
\left[k_{c}-\omega^{2} m_{\mathrm{eff}}+j \omega \gamma_{c}+K^{*}\right] \tilde{u}_{1}=\left[c_{1}\left(\omega^{2} m_{\mathrm{eff}}-j \omega \gamma_{c}\right)\left(1+B_{f}^{*}\right)-K^{*} / \psi_{1}(L)\right] \tilde{z}_{b}
$$


where $K^{*}=k_{\text {int }}+j \omega \gamma_{\text {int }}$, and we arrive at:

$$
\frac{A e^{j \phi}}{A_{b} e^{j \phi_{b}}}=\frac{c_{1} G^{*}\left(1+B_{f}^{*}\right)-K^{*} / \psi_{1}(L)}{k_{c}-G^{*}+K^{*}}
$$

Summarizing our main results, we have obtained the following expressions:

$$
\begin{aligned}
& \hat{A}=\frac{A e^{j \phi}}{A_{\infty} e^{j \phi_{\infty}}}=\frac{\left(c_{1} G^{*}\left(1+B_{f}^{*}\right)-K^{*} / \psi_{1}(L)\right)\left(k_{c}-G^{*}\right)}{c_{1} G^{*}\left(1+B_{f}^{*}\right)\left(k_{c}-G^{*}+K^{*}\right)} \\
& \hat{A}_{s}=\frac{A_{s} e^{j \phi_{s}}}{A_{\infty} e^{j \phi_{\infty}}}=\frac{k_{c}-G^{*}}{\bar{k}_{c}-G^{*}} \frac{G^{*}\left(g_{1}+\bar{c}_{1} B_{f}^{*}\right)+\lambda\left(\bar{k}_{c}-G^{*}\right)}{c_{1} G^{*}\left(1+B_{f}^{*}\right)}
\end{aligned}
$$

from which the unknowns $B_{f}^{*}, A_{b} e^{j \phi_{b}}$ and $K^{*}$ can be determined using the experimentally obtained $A e^{j \phi}, A_{\infty} e^{j \phi_{\infty}}$ and $A_{s} e^{j \phi_{s}}$. The other quantities are known from calculation.

First we determine $B_{f}^{*}$ :

$$
B_{f}^{*}=\frac{c_{1} G^{*}\left(\bar{k}_{c}-G^{*}\right) \hat{A}_{s}-\left(k_{c}-G^{*}\right)\left(G^{*} g_{1}+\lambda\left(\bar{k}_{c}-G^{*}\right)\right)}{\bar{c}_{1} G^{*}\left(k_{c}-G^{*}\right)-c_{1} G^{*}\left(\bar{k}_{c}-G^{*}\right) \hat{A}_{s}}
$$

Next we determine $K^{*}$ from Eqs. (a26) and (a27):

$$
K^{*}=\frac{G^{*}\left(k_{c}-G^{*}\right)\left(1+B_{f}^{*}\right)(1-\hat{A})}{G^{*}\left(1+B_{f}^{*}\right) \hat{A}+\left(k_{c}-G^{*}\right) /\left(c_{1} \psi_{1}(L)\right)}
$$

Eqs. (a28) and (a29) are our final result. From numerical calculations, we get the values for the four coefficients, $c_{1}=2.1556, \psi_{1}(L)=0.7265$, $g_{1}=-2.9128$, and $\bar{c}_{1}=-4.9133$.

\section{A.2 Supplementary Information}

\section{Experimental settings, calibration of tip-substrate dis- tance}

In Table S2 the experimental settings are given for all three types of measurements: DLVO force, hydrodynamic dissipation, and hydration force, which are done with both AMS and TNS.

Figures S1 and S2 show how the tip-substrate distance is determine from the static deflection curves. 
Chapter 4. Amplitude modulation Atomic Force Microscopy, is acoustic driving in liquid quantitatively reliable?

\begin{tabular}{|c|c|c|c|c|c|c|}
\hline Type of interaction & \multicolumn{2}{|c|}{ DLVO } & \multicolumn{2}{|c|}{ Hydro. diss. } & \multicolumn{2}{|c|}{ Hydration } \\
\hline Method & AMS & TNS & AMS & TNS & AMS & TNS \\
\hline Approach rate $(\mathrm{nm} / \mathrm{s})$ & 100 & 5 & 100 & 3 & 1 & 0.3 \\
\hline Retract rate $(\mathrm{nm} / \mathrm{s})$ & 100 & 100 & 100 & 60 & 1 & 6 \\
\hline Ramp distance (nm) & 50 & 50 & 100 & 30 & 5 & 3 \\
\hline Number of approaches & 52 & 9 & 10 & 3 & 81 & 30 \\
\hline Amplitude* (pm) & 205 & 43 & 380 & 32 & 120 & 65 \\
\hline
\end{tabular}

Table S2: Experimental settings of all three measurements with AMS and TNS. * for TNS Amplitude means: rms value.

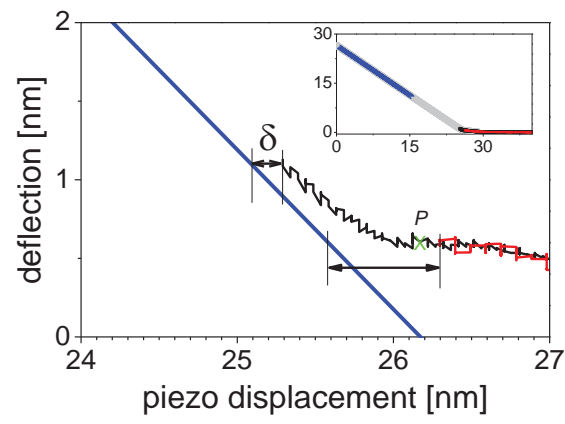

Figure S1: Determination of the tip-substrate separation from the measured static deflection against piezo displacement. The data is from the DLVO force measurement. The straight line (blue) is the linear fit of deflection against piezo displacement (for static deflection from $10 \mathrm{~nm}$ to $26 \mathrm{~nm}$, as shown in the inset). Then the tip-substrate separation is the horizontal distance between each data point and the linear fit line. For TNS approaches (black), the minimum separation $\delta$, shown as a back double arrow, is $0.20 \mathrm{~nm}$, for AMS (red) $0.68 \mathrm{~nm}$. 


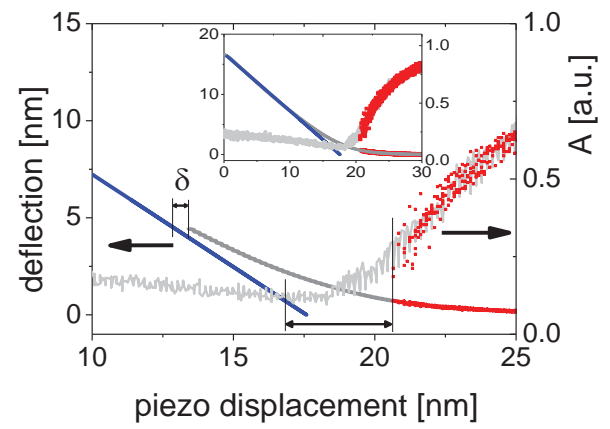

Figure S2: Determination of the tip-substrate separation for the measurement of hydrodynamic dissipation. The straight line (blue) is the linear fit of deflection against piezo displacement (for static deflection from $6 \mathrm{~nm}$ to $16 \mathrm{~nm}$, as shown in the inset). The amplitude response measured simultaneously are plotted in gray. For TNS approaches (dark grey), the minimum separation $\delta$, shown as a double arrow, is $0.50 \mathrm{~nm}$, for AMS (red) $3.70 \mathrm{~nm}$.

\section{Charge regulation and DLVO force calculation}

For the formulation of charge regulation (CR) boundary on silica surface, we adopt the Stern layer model of electric double layer with consideration of two surface reactions [3]: (1) deprotonation of surface silanol group $\mathrm{SiOH} \leftrightharpoons \mathrm{SiO}^{-}+H^{+}$with $p K_{H}$ on 0 plane; (2) adsorption of electrolyte cations $\mathrm{SiONa} \leftrightharpoons \mathrm{SiO}^{-}+\mathrm{Na}^{+}$with $p K_{N a}$ on s plane (or Stern plane). In addition, for the conservation of silanol group on silica surface, one can write $\left\{\mathrm{SiO}^{-}\right\}+\{\mathrm{SiOH}\}+\{\mathrm{SiONa}\}=\Gamma$, where represents the surface site density and is the site density of silanol group originally present on silica surface. Then the site densities of three surface species can be solved from the follow matrix equation

$$
\left(\begin{array}{ccc}
1 & 1 & 1 \\
-\left[H^{+}\right]_{0} & K_{H} & 0 \\
-\left[N a^{+}\right]_{s} & 0 & K_{N a}
\end{array}\right)\left(\begin{array}{c}
\left\{S_{i O}-\right\} \\
\{S i O H\} \\
\{S i O N a\}
\end{array}\right)=\left(\begin{array}{c}
\Gamma \\
0 \\
0
\end{array}\right)
$$

where $K_{H}=10^{-p K_{H}}$ and $K_{N a}=10^{-p K_{N a}}$ are equilibrium constants of the aforementioned two surface reactions. It should be noted that solutions of three surface species involve the volume proton concentration at 0 plane $\left(\left[\mathrm{H}^{+}\right]_{0}\right)$ and the volume cation concentration at the s plane $\left(\left[\mathrm{Na}^{+}\right]_{s}\right)$ 

is acoustic driving in liquid quantitatively reliable?

which can be evaluated according to the Boltzmann distribution as $\left[\mathrm{H}^{+}\right]_{0}=$ $\left[\mathrm{H}^{+}\right]_{\infty} e^{-e \psi_{0} / k_{B} T}$ and $\left[\mathrm{Na}^{+}\right]_{s}=\left[N a^{+}\right]_{\infty} e^{-e \psi_{s} / k_{B} T}$, respectively. The subscripts 0, s and $\infty$ denote surface of silica, Stern plane and the bulk solution (far from the solid surface), respectively. The surface charge is neutralized by the charge inside diffuse part of electric double layer, or the diffuse layer charge $\sigma_{d}$, which implies $\sigma_{d}=-e\left\{\mathrm{SiO}^{-}\right\}$.

To formulate a CR boundary condition, we express the surface charge density, $\sigma_{d}$, in terms of $\psi_{s}$, the potential at the s plane. Therefore we relate $\psi_{0}$ with the surface charge $\sigma_{0}$ as $\psi_{0}-\psi_{s}=\sigma_{0} / C_{s}$

(where $C_{s}$ denotes the capacitance of the Stern layer) while the charge density at the 0 plane $\sigma_{0}=-e\left\{S_{i O}^{-}\right\}-e\{S i O N a\}$. With this we solve the matrix equiation given above, obtaining the CR boundary condition $\sigma_{d}=\mathcal{F}_{c r}\left(\psi_{s},[N a C l]_{\infty}, p H, \Gamma, C_{s}, p K_{H}, p K_{N a}\right)$, where $p H=-\log _{10}\left[H^{+}\right]_{\infty}$. Table S3 summarizes the parameters used in the DLVO force calculation.

\begin{tabular}{r|l}
\hline Deprotonation $\mathrm{pK}_{\mathrm{H}}$ & $6.90[3]$ \\
Cation adsorption $\mathrm{pK}_{\mathrm{Na}}$ & $1.65[3]$ \\
Stern layer capacitance $C_{S}$ & $2.9 \mathrm{~F} / \mathrm{m}^{2}[4]$ \\
Site density of silanol group $\Gamma$ & $8 / \mathrm{nm}^{2}[4]$ \\
Hamaker constant $A_{H}$ & $0.65 \times 10^{-20}[5,6]$ \\
Stern layer thickness $d_{S}$ & $0.4 \mathrm{~nm}[7,8]$ \\
pH of the solution & 9 \\
Interaction area $S$ & fitting parameter \\
\hline
\end{tabular}

Table S3: Silica parameters used in the DLVO force calculation with charge regulation

\section{Influence of uncertainty in $A_{s} / A_{\infty}$}

From the hydrodynamic dissipation measurement with a colloidal probe, the AM calibration approaches are shown in Fig. S2. The amplitude does not reach a plateau when the cantilever is in contact with the substrate, which is unlike the phase. This disallows us to determine the exact value of the amplitude on surface, $A_{s}$. We test three values for $A_{s} / A_{\infty}$, which are 0.118 (the minimum from calibration approaches), 0.158 (used for the results in the main text) and 0.263 (above this, we get unrealistically negative dissipation). The results are shown in Fig. S3. 


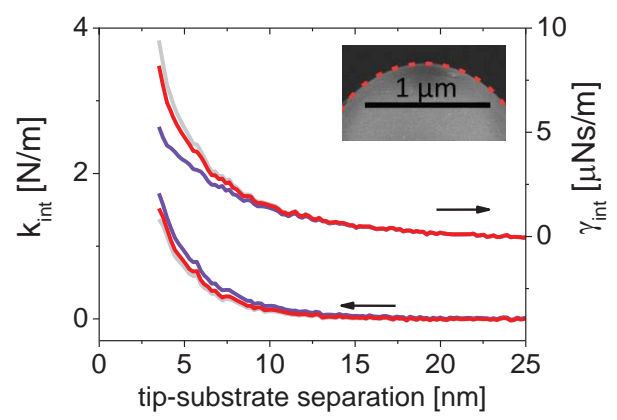

Figure S3: Results from force inversion with different values for $A_{s} / A_{\infty}$, 0.118 (gray), 0.158 (red), and 0.263 (purple).

\section{Comparison between the static deflection and TNS in the hydration force measurement}

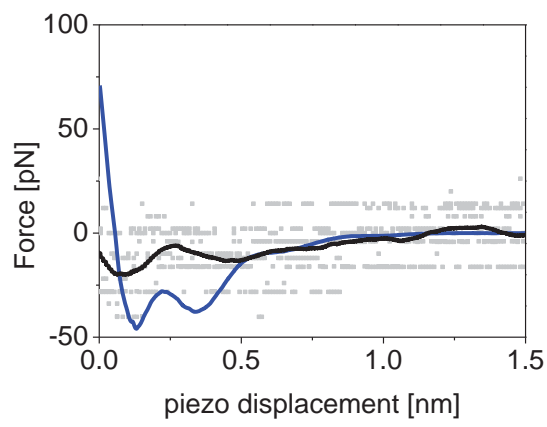

Figure S4: Forces obtained from the static deflection (raw data in grey, average in black) and TNS (in blue) in the hydration force measurement.

1. D. Kiracofe and A. Raman; Nanotechnology 22 (2011) 485502

2. X. Xu and A. Raman; J. Appl. Phys. 102 (2007) 034303

3. C. Zhao, D. Ebeling, I. Siretanu, D. van den Ende and F. Mugele; (2015) to be submitted

4. T. Hiemstra, W. H Van Riemsdijk and G. H Bolt; J. Colloid Interface Sci. 133 (1989) 91 
Chapter 4. Amplitude modulation Atomic Force Microscopy, is acoustic driving in liquid quantitatively reliable?

5. D. Ebeling, D. Van Den Ende and F. Mugele; Nanotechnology 22 (2011) 305706

6. M. Dishon, O. Zohar and U. Sivan; Langmuir 25 (2009) 2831

7. T. Hiemstra, H. Yong and W. H Van Riemsdijk; Langmuir 15 (1999) 5942

8. J. Newman and K. E Thomas-Alyea; Electrochemical Systems (2004) (John Wiley \& Sons) 


\section{Bibliography}

[1] O'Shea S J and Welland M E 1998 Langmuir 144186

[2] Ebeling D, van den Ende D and Mugele F 2011 Nanotechnology 22 305706

[3] Siretanu I, Ebeling D, Andersson M P, Stipp S L S, Philipse A, Cohen Stuart M, van den Ende D, and Mugele F 2014 Sci. Rep. 44956

[4] Garcia R and Herruzo E T 2012 Nature Nanotechnology 7217

[5] Sader J E and Jarvis S P 2006 Phys. Rev. B 74195424

[6] de Beer S, van den Ende D and Mugele F 2011 J. Phys.: Condens. Matter 2311206

[7] Xu X and Raman A 2007 J. Appl. Phys. 102034303

[8] Patil S, Matei G, Oral A and Hoffmann P M 2006 Langmuir 226485

[9] Umeda N, Ishizaki S, and Uwai H 1991 J. Vac. Sci. Technol. B 91318

[10] Labuda A, Kobayashi K, Kiracofe D, Suzuki K, Grutter P H and Yamada H 2011 Aip Advances 1022136

[11] de Beer S, van den Ende D and Mugele F 2010 Nanotechnology 21 325703

[12] Maali A, Hurth C, Cohen-Bouhacina T, Couturier G and Aimé J P 2006 Appl. Phys. Lett. 8808615

[13] Putman C A J, Van der Werf K O, De Grooth B G, Van Hulst N F and Greve J 1983 Phys. Rev. Lett. 51 1336-1339

[14] Kiracofe D and Raman A 2011 Nanotechnology 22485502

[15] de Beer S, den Otter W K, van den Ende D, Briels W J and Mugele F 2012 Euro Phys. Lett. 9746001

[16] Liu F, de Beer S, van den Ende D and Mugele F 2013 Phys. Rev. E 87 062406

[17] Israelachvili J 1991 Intermolecular and Surface Forces 2nd ed (Academic press, London) 
[18] Iler R K 1979 The Chemistry of Silica (John Wiley and sons, New York)

[19] Zhao C, Ebeling D, Siretanu I, van den Ende D, and Mugele F 2015 Nanoscale Doi: 10.1039/C5NR05261K

[20] Kaggwa G B, Kilpatrick J I, Sader J E and Jarvis S P 2008 Appl. Phys. Lett. 93011909

[21] Kilpatrick J I, Loh S H and Jarvis S P 2013 J. Am. Chem. Soc. 135 2628

[22] Herruzo E T, Asakawa H, Fukuma T and Garcia R 2013 Nanoscale 7 2678

[23] Fukuma T, Higgins M J and Jarvis S P 2007 J. Chem. Phys. 923603

[24] Argyris D, Phan A, Striolo A, and Ashby P D 2013 J. Phys. Chem. C 11710433

[25] Labuda A, Kobayashi K, Suzuki K, Yamada H and Grütter P 2013 Phys.Rev. Lett. 110066102

[26] Landau L D, Lifshitz E M 1987 Fluid Mechanics (Pergamom Press, Oxford)

[27] Butt H J and Jaschke M 1995 Nanotechnology 61

[28] Mugele F, Bera B, Cavalli A, Siretanu I, Maestro A, Duits MHG, CohenStuart M, van den Ende D, Stocker I, and Collins I 2015 Sci. Rep. 5 10519

[29] Zhmud B V, Meurk A and Bergstrom L 2000 Colloids and Surfaces A: Physicochem. Eng. Aspects 1643

[30] Rice C L, Whitehead R 1965 J. Phys. Chem. 694017

[31] Israelachvili J N 1986 J. Colloid and Interface Sci. 110263

[32] Maali A, Cohen-Bouhacina T, Couturier G and Aimé J-P 2006 Phys. Rev. Lett 96086105

[33] Katan A J, van Es M H and Oosterkamp T H 2009 Nanotechnology 20 2678

[34] Katan A J $2008 \mathrm{PhD}$ thesis, University of Leiden

[35] de Beer S, van den Ende D and Mugele F 2008 Appl. Phys. Lett. 93 253106

[36] Khan S H, Matei G, Patil S and Hoffmann P M 2010 Phys. Rev. Lett 105106101

[37] Watkins M, Berkowitzb M L and Shlugerac A L 2011 Phys. Chem. Chem. Phys. 1312584

[38] Leng Y, Cummings P T 2006 J. Chem. Phys. 124074711

[39] de Beer S, den Otter W K, van den Ende D, Briels W J and Mugele F 2012 Tribol. Lett. 481 


\section{Chapter 5}

\section{Viscous dissipation in overlapping electric double layers}

We investigate the enhancement of the hydrodynamic dissipation in a flow through the overlapping electric double layers, which is between tip and substrate in Atomic Force Microscopy. We observe that the dissipation enhancement is correlated with the surface charge at tip and substrate, and it is maximal for a tip-substrate distance of about the Debye length. We compare our findings with a simple lubrication model, where the excess ion distribution in the electrolyte film between tip and substrate is considered to be trapped and modeled as body force, acting on the flow and therefore causing an enhancement of the dissipation. This model describes our experimental findings qualitatively. The order of magnitude is also quantitatively in agreement with the experiments, but the calculated length scale at which maximum enhancement occurs is much larger than the observed length scale. For large distances, the enhancement scales approximately with the inverse of the ionic strength as expected. However, near close contact, the behavior is not understood. Considering also viscosity enhancement due to the strong local electric fields in the double layer does not solve this issue. The fact that the issue remains unresolved may be due to the poorly understood effects of ionic transport and hydration in the Stern part of the electric double layer. 


\subsection{Introduction}

Most solid surfaces become charged upon contact with aqueous solutions. Examples include mineral surfaces, polymers, metal oxides, and biological materials [1-4]. The surface charge is screened by the counter ions present in the electrolyte that are attracted towards the surface. The resulting ionic distribution is called the electric double layer (EDL), a combination of a compact Stern layer and a diffuse layer [5]. In various disciplines of science and technology, including electrochemistry, [6] colloid science [5] and nanofluidic applications [7], the understanding of the phenomena occurring in the EDL is very important. In particular, understanding the coupling between electrostatics and hydrodynamics is key to interpreting various measurements, including streaming potential, zeta potential, and viscosity of suspensions. Great efforts have been made to understand the properties of the EDL, including EDL mediated interfacial forces and in particular dynamic effects such as enhanced viscous dissipation in the EDL. Nevertheless, the coupling between hydrodynamics and electrostatics in the EDL remains unresolved.

The charge distribution in the diffuse layer is conventionally described by the Poisson-Boltzmann (PB) equation. The PB theory, as a mean-field approach, is a convenient tool and it has been widely applied for about a century. One of the most prevailing applications is the prediction of the EDL force. The EDL force and the van der Waals force constitute the wellknown DLVO force, named after the pioneers Derjaguin, Landau, Verwey and Overbeek [1].

DLVO theory is arguably the most successful theory in soft matter interfacial science. In colloid science, the DLVO forces are able to explain, often even quantitatively, various phenomena like the stability, aggregation and adsorption behavior of colloidal suspensions [5]. At present, the atomic force microscope and the surface force apparatus [1] are widely used to probe the DLVO force between two charged substrates in an electrolyte environment. The force-distance curves are measured with Angstrom distance resolution and pico-Newton force resolution. The surface charge density is extracted by fitting the force-distance curves to the DLVO theory. In most cases, the theory predicts the measured forces very well [8-10]. However, it fails at small separations of a few molecular layers. In this regime, the discrete nature of the molecules comes into the picture and therefore non-DLVO forces, for instance solvation forces (e.g. hydration force for water), are more dominant [1].

It is not surprising that continuum theories break down near a substrate. Molecular dynamics (MD) simulations and density functional theory (DFT), 
have shed light on the complexity of the interface, which is reflected in the position dependent density, viscosity, and electric permittivity [11]. It also leads to ion specific effects $[12,13]$. It is of vast interest to know how to connect the non-continuum effects near the substrate to the bulk phenomena and to what extent continuum theories are applicable [11,14]. A good example is the electroviscous effect, an unresolved issue in colloid science [15], that has been revisited recently in the context of nanofluidics [16].

Consider a channel with charged walls that is filled with electrolyte. When a hydrostatic pressure gradient is applied, the flow will carry the mobile screening charges in the diffuse part of the EDL downstream. As a result, a streaming current (and potential) is produced. This energy conversion scheme enables the conversion of pressure and fluid flow to electrical energy. It is attractive because it seems environmental friendly. Therefore, it has already been a 'hot' topic in nanofluidics research for a decade [16-21]. In the classical picture, the pressure-driven streaming current is balanced by an electric conduction current. In analogy with a battery in a closed circuit, the battery produces current/potential, but inside the battery the net current must be zero. The conduction ions will drag the solvent with it, which is called electro-osmosis. The opposite electro-osmotic flow effectively reduces the flow rate and leads to an increase of the apparent viscosity of the solution. This phenomenon is known as the electro-viscous effect [22].

The electro-viscous effect was originally introduced in colloid science when researchers tried to explain the increment in the viscosity of suspensions of charged particles [23]. The screening charges around the particle affect the flow field and thereby enhance the dissipation. Therefore, the viscosity of a diluted suspension containing charged particles, is strongly underestimated by the well-known Einstein relation for hard sphere suspensions [15]. Due to the complex coupling between electrostatics and hydrodynamics when considering the flow of a suspension of charged colloids, adequate theory to describe the limited experimental data is still lacking [15]. In nanofluidic channels, the degree of complexity is largely reduced, mainly because the laminar flow profile is straightforward. Nevertheless, discrepancies between theory in the $\mathrm{PB}$ framework and the experiments are frequently reported [16, 24-26].

There are several hurdles to take before one can get a sound quantitative picture of electro-viscous dissipation, as there is the determination of i) the surface charge (or potential), ii) the hydrodynamic boundary conditions, iii) the mobility of the ions and iv) the ionic charge distribution in the electrolyte near the charged surfaces. There exists an intricate interplay between these parameters, as will be shown below.

First of all, we consider the surface charge (or potential). It is an im- 
portant parameter for predicting among others the EDL force, but it is not easily accessible. Conventionally, it is measured via titration or electrokinetic techniques [5], including electrophoresis [27]. In electrophoresis, the well-known Smoluchowski theory, still in the framework of the PB theory, is widely applied for determination of $\zeta$ potentials at the shear plane [27]. The shear plane is conceptually between the hydrodynamic stagnant layer and the hydrodynamic mobile layer occurring in the flow over a charged surface caused by an external electric field. Although the concept of a shear plane is widely applied in the colloid community, [28] there is no definitive way to locate it. The measured $\zeta$ potentials for supposedly the same interfaces often vary considerably, depending on the choice of measuring technique or theory [29].

Secondly, the hydrodynamic boundary conditions are an important factor. It has been shown theoretically that for a slip boundary, in the framework of the PB theory, the electro-viscous effect is larger compared to the case of no-slip boundary [30]. It is because for a slip boundary the shear near the boundary is strongly reduced, and therefore the overall viscous dissipation decreases, while the streaming potential is amplified [31].

Thirdly, the dynamic properties of the ions at charged interfaces has not been resolved. The concept of a dynamic Stern layer has been proposed for remedying the discrepancies between the $\zeta$ potentials measured in different studies [27]. It assumes that the ions between the substrate and the shear plane are mobile. But it is not clear to what extent their mobility differs from bulk mobility. Note that the majority of the counter ions reside below the shear plane. The ion densities below the shear plane are appreciably higher than in the diffuse layer. Hence, ion-ion correlations and ion-crowding effects may come into play [32].

Fourthly, the properties of water near the substrate are modified by the ion distribution and the electric field strength at the substrate, which are not captured by a continuum theory. It is well-established that the water molecules, due to their strong dipole moment, are aligned along the electric field [33]. As a result, the permittivity of the water goes down, which is called permittivity saturation [34]. Moreover, because the rotational orientation of water molecules gets trapped in a strong electric field, the resistance to shear flow increases with increasing field strength and therefore the viscosity is increased, $\eta=\eta_{0}\left(1+f_{v e} E^{2}\right)$, where $\eta_{0}$ is the bulk viscosity of water, $f_{v e}$ is the visco-electric coefficient and $E$ is the electric field strength. This enhancement is known as the visco-electric effect. It has been tested for various polar organic solvents with different molecular dipole moments [35]. Based on the extrapolation from these data, Lyklema and Overbeek estimated the 
visco-electric coefficient $f_{v e}$ for water as $10^{-15}(\mathrm{~m} / \mathrm{V})^{2}$. Although it is highly desirable to measure $f_{v e}$ directly, because of its relevance in electrokinetic transport phenomena [34], to the best of our knowledge, there are no definitive measurements of $f_{v e}$ after the pioneering work by Hunter [36].

The electroviscous effect was investigated by measuring the pressure drop across a nano/micro channel filled with electrolyte solution. A discrepancy has been found between theoretical prediction and observation, e.g. the measured enhancement is often larger than predictions based on the PB theory $[16,37]$. Several groups studied the electro-viscous effect by monitoring the spontaneous filling of nanocapillaries by an electrolyte solution due to capillary pressure [24-26]. From the filling speed, the apparent viscosity is extracted, using the classical Washburn formula [38]. From the limited number of studies, controversial findings have been reported. Tas and van den Berg et al. reported an increase by $20 \sim 30 \%$ of apparent viscosity [24]. Later they reported an even larger enhancement, which was partially attributed to the possible existence of a solidified water layer on the channel walls [25]. Another group reported an enhancement by $1 \%$ at most in the apparent viscosity for all practical cases [26]. There are two caveats with this method. The filling process is affected by unpredictable pinning and depinning at the contact line due to surface roughness and heterogeneity [39]. Moreover, the emergence of trapped air bubbles can partially block the flow. Nanochannels are often fabricated from silica by chemically etching. It causes a surface roughness, which depends on the etching time [16]. To fabricate distinct and well-defined nanochannels is still a major challenge in nanofluidics [40].

The just described complexity of electrolyte flow over charged substrates motivated us to explore alternative routes to investigate the electro-hydrodynamics. Here we propose a new approach: nanoscopic AFM force spectroscopy. We use an AFM to study viscous dissipation in overlapping EDLs, e.g. dissipation in a thin film of electrolytes confined between two charged surfaces. The two charged surfaces, on one side the sample substrate and on the other side the AFM tip, consist both of silica. The physical and chemical properties of silica surfaces are well documented. A silica surface is hydrophilic, preventing water to slip over it [41]. The (negative) surface charge of silica is controlled by its surface chemistry and can be regulated by varying the $\mathrm{pH}$ and concentration of electrolyte in the bulk.

An AFM based study has the following advantages. First of all, the system is well controlled. The surface roughness of the substrate, thermally grown silica, is on the order of an Angstrom. Secondly, the conservative DLVO force and the dissipation can be measured simultaneously as a func- 


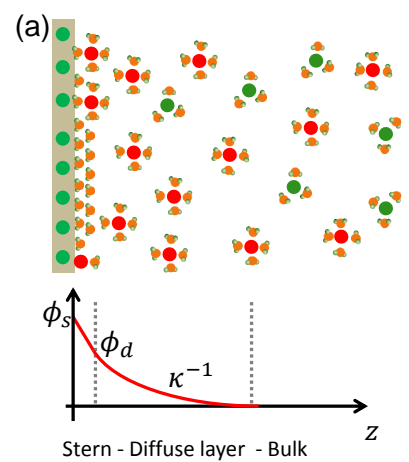

(b)

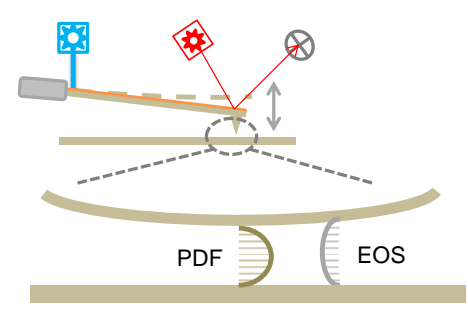

Figure 5.1: (a) A sketch of the electric double layer. (b) Schematic presentation of the experimental set-up. The cantilever is oscillated photo-thermally by a blue laser spot with a periodically varying intensity. The deflection of the cantilever is detected by reflecting the red laser onto a four quadrant detector. The electrolyte is confined between the tip of an AFM cantilever and the silica substrate. The oscillating tip generates two types of flows: a pressure driven flow (PDF) and an oppositely directed electro-osmotic flow (EOS).

tion of tip-substrate separation by ramping the oscillating AFM tip up and down to the substrate. From the electrostatic force-distance curves, we are able to evaluate the distance-dependent surface charge by analyzing the ionic concentration profiles and the charge-potential relation at both substrates. It enables us to evaluate the viscous dissipation in relation to the relevant electro-chemical parameters that characterize the overlapping double layers.

We observe a strong correlation between surface charge and enhanced dissipation. Using the surface charge and potential distribution as obtained from our DLVO analysis, we consider two contributions to the electro-viscous effect: the trapping of ions in the electrolyte film and the viscosity enhancement due to the locally very high electric field strength. The measured dissipation enhancement is qualitatively in agreement with the prediction of the simple model, based on PB theory and considering the excess charge trapped in the electrolyte film. Quantitative differences can possibly be attributed, amongst others, to surface conduction, reduced ion diffusivity or electric field enhanced viscosity. 


\subsection{Methods}

We perform measurements with two AFM probes of different tip geometry, a colloidal probe and a nanoscale tip. The nanoscale tip is flattened by rubbing against the substrate of the sample (see Chapter 4 for details), to enhance both the stability of the tip and the strength of the interaction forces. The measurement procedures are only different in minor details. Below we illustrate the methods with the measurements conducted with a colloid probe.

\subsubsection{AFM force spectroscopy with photo-thermal ex- citation}

Using amplitude modulation (AM) AFM we probe the amplitude and phase response of the cantilever tip. We drive the bending of the cantilever by locally heating it with a laser beam, using the so-called photothermal drive principle [42]. The laser intensity $I$ varies harmonically as $I=I_{0}(1+$ $\exp (j \omega t))$ where $\omega$ is the driving frequency and $I_{0}$ the amplitude of the oscillating intensity. Due to the oscillating temperature field over the cantilever, resulting from this heating process, the relation between force $F$ and tip displacement $z$ is not simply given by $F=k_{c} z$, where $k_{c}$ is the effective stiffness of the cantilever, but by:

$$
F=k_{c}\left(z-z_{T}\right)
$$

where $z_{T}$ is the zero-load-deflection due to the temperature enhancement along the beam caused by the laser radiation with intensity $I$. According to linear response theory, $z_{T}$ can be written, for small thermal variations, as $z_{T}(t)=\int_{0}^{\infty} A_{T}\left(t^{\prime}\right) I\left(t-t^{\prime}\right) d t^{\prime}$ or in the frequency domain as $z_{T}(\omega)=A_{T}^{\star}(\omega) I_{0}$. The amplitude and phase of the tip displacement, $z(\omega)=A e^{j \phi}$, are measured as a function of the tip-substrate distance $h$, to probe the influence of the tip substrate interaction on the oscillation behavior of the cantilever tip. To analyze the measured response in terms of the tip substrate interaction, we model the motion of the cantilever tip as a simple harmonic oscillator (SHO) [43-47]:

$$
m^{*} \ddot{z}+\gamma_{c} \dot{z}+k_{c} z=k_{c} z_{T}(t)+F_{t s}(h+z, \dot{z})
$$

where $m^{*}=k_{c} / \omega_{0}^{2}$ the effective mass of the cantilever including the added mass due to the surrounding liquid, $\omega_{0}$ the resonance frequency, $\gamma_{c}=k_{c} /\left(\omega_{0} Q\right)$ its damping coefficient, $Q$ the quality factor of the oscillator and $F_{t s}$ the tip-sample interaction force. When the amplitude of the tip oscillation is 
sufficiently small, compared to the characteristic interaction length, the tipsample interaction force can be linearized as:

$$
F_{t s}(h+z, \dot{z})=F_{t s}(h, 0)-k_{i n t} z-\gamma_{i n t} \dot{z},
$$

where $k_{\text {int }}=-\partial F_{t s} / \partial h$ is the interaction stiffness, $\gamma_{\text {int }}$ the interaction damping coefficient and $F_{t s}(h, 0)$ the equilibrium force at distance $h$. In this study, the linearization is justified because the oscillation amplitude is less than 1 $\mathrm{nm}$, which is much smaller than the characteristic length of the interaction force, as can be judged from the amplitude and phase curves in Fig. 5.3. Substituting Eq. (5.3) into Eq. (5.2), we can rewrite Eq. (5.2) in the frequency domain as:

$$
\left(k_{c}-m^{\star} \omega^{2}+j \gamma_{c} \omega\right) z(\omega)=k_{c} I_{0} A_{T}^{\star}-\left(k_{\mathrm{int}}+j \gamma_{\mathrm{int}} \omega\right) z(\omega)
$$

Writing $z(\omega)=A e^{j \phi}$ and $A_{T}^{\star}=A_{T} e^{j \phi_{T}}$ the transfer function becomes:

$$
\frac{A e^{j \phi}}{I_{0}}=\frac{k_{c} A_{T} e^{j \phi_{T}}}{k_{c}-m^{\star} \omega^{2}+j \gamma_{c} \omega+k_{\mathrm{int}}+j \gamma_{\mathrm{int}} \omega}
$$

\section{Calibration and force inversion}

Before we can use Eq. (5.5) to determine $k_{\text {int }}$ and $\gamma_{\text {int }}$ from $A e^{j \phi}$, we have to calibrate the cantilever parameters $k_{c}, \gamma_{c}$ and $m^{\star}$. The stiffness $k_{c}$ is obtained from the thermal noise spectrum of the cantilever [48] when the cantilever is not in contact with the substrate, i.e. $h>1 \mu \mathrm{m}$. The mass $m^{\star}$ and the damping coefficient $\gamma_{c}$ are obtained from the resonance frequency $\omega_{0}$ and the quality $Q$ of the oscillator under liquid. The hydrodynamic loading on the cantilever beam varies with tip-substrate separation, but it is constant over the distances $150 \mathrm{~nm}<h<1 \mu \mathrm{m}$ [49]. In this range $h$ is much smaller than the height of the tip cone. Therefore, $\omega_{0}$ and $Q$ are calibrated using the thermal noise spectrum measured at a distance of $140 \mathrm{~nm}$ from the surface. At this separation, the tip-substrate interaction is supposed to be negligible.

In our case (tip $R=1080 \mathrm{~nm}$, water viscosity $0.8 \mathrm{mPa} \cdot \mathrm{s}$ at the measurement temperature $30^{\circ} \mathrm{C}$ ), the damping under the tip is approximately 100 $\mathrm{nNs} / \mathrm{m}$ at $140 \mathrm{~nm}$ (see Eq. (5.19)), which is much smaller than the damping of the cantilever beam, typically around $5 \%$ of $\gamma_{c}$. Nevertheless, for the sake of accuracy, in the following analysis the minor damping $\gamma_{\text {int }}$ at the largest separation is taken into account.

The resonance frequency $\omega_{0}$ and the quality factor $Q$ are obtained by fitting the power spectral density of the SHO to the measured thermal spectral 
density. Figure 5.2 shows a typical noise spectrum together with the photothermal response function, both measured at a distance of $140 \mathrm{~nm}$. We can observe a slight difference between both spectra. This difference is caused by the thermal driving coefficient $A_{T}^{\star}$ in Eq. (5.5) which is a function of the driving frequency $\omega$.

Once we have determined the cantilever parameters we can use Eq. (5.5) to derive the force inversion equations. Because the thermal driving coefficient $A_{T}^{\star}$ is not known, we also measure $A e^{j \phi}$ at a distance of $140 \mathrm{~nm}$ from the substrate, where both $k_{\text {int }}$ and $\gamma_{\text {int }}$ are zero. We denote this value as $A_{\infty} e^{j \phi_{\infty}}$. Considering the ratio $A / A_{\infty} e^{j\left(\phi-\phi_{\infty}\right)}$ we obtain with Eq. (5.5):

$$
\frac{A e^{j \phi}}{A_{\infty} e^{j \phi_{\infty}}}=\frac{k_{c}-m^{\star} \omega^{2}+j \gamma_{c} \omega}{k_{c}-m^{\star} \omega^{2}+j \gamma_{c} \omega+k_{\text {int }}+j \gamma_{\text {int }} \omega}
$$

from which we obtain the force inversion formulas $[10,45]$ :

$$
k_{\text {int }}=k_{c}\left[1-\left(\frac{\omega}{\omega_{0}}\right)^{2}\right] \frac{A_{\infty} \cos \left(\phi-\phi_{\infty}\right)-A}{A}+\gamma_{c} \omega \frac{A_{\infty} \sin \left(\phi-\phi_{\infty}\right)}{A}
$$

and

$$
\gamma_{\mathrm{int}}=\gamma_{c} \frac{A_{\infty} \cos \left(\phi-\phi_{\infty}\right)-A}{A}-k_{c}\left[1-\left(\frac{\omega}{\omega_{0}}\right)^{2}\right] \frac{A_{\infty} \sin \left(\phi-\phi_{\infty}\right)}{\omega A}
$$

\subsubsection{Materials}

For the AFM experiments, we used a silicon wafer with a thermally grown silica layer of $90 \mathrm{~nm}$ thickness on top. The sample is firmly glued, using epoxy, to a stainless puck, which is magnetically clamped to the piezo stage of the AFM. The RMS roughness of the surface is approximately $0.2 \mathrm{~nm}$ for a $1 \mu m \times 1 \mu m$ area. Prior to use, the substrate is rinsed with consecutively isopropanol, ethanol and ultrapure water (MilliQ Inc.) in a sonication bath for 10 min. After drying with a jet of $\mathrm{N}_{2}$, it is exposed to a plasma of residual air (Harrick Plasma) for $30 \mathrm{~min}$. The cantilevers are cleaned in a similar manner without using a sonication bath. The solutions are prepared by dissolving $\mathrm{NaCl}$ (Sigma Aldrich) in ultrapure water. The $\mathrm{pH}$ of the electrolyte solution is controlled by adding $\mathrm{NaOH}$ to the solution. The resulting $\mathrm{pH}$ value is measured using a probe (HI 1053, HANNA Instruments). For our measurements we used 5 different solutions, aiming for: $\mathrm{pH} 5.7 / 1 \mathrm{mM}, \mathrm{pH}$ 9.3/0.1 mM, pH 9.3/1 mM, pH 9.3/10 mM, and for reference purposes $\mathrm{pH}$ $5.7 / 100 \mathrm{mM}$. To obtain the desired concentrations, first a stock solution of 1 $\mathrm{M} \mathrm{NaCl}$ was used. From the stock solution, $10 \mathrm{ml}$ amounts of 100, 10, 1 and 
$0.1 \mathrm{mM} \mathrm{NaCl}$ were obtained by repeated dilution. The $\mathrm{pH}$ of these diluted solutions was 5.7. To set the $\mathrm{pH}$ to $9.3,10 \mu \mathrm{l}$ of $0.1 \mathrm{M} \mathrm{NaOH}$ is added to $10 \mathrm{ml} \mathrm{NaCl}$ solutions. As we will show below, the lowest concentration turns out to be 0.2 in stead of $0.1 \mathrm{mM}$, most probably due to slight contamination of the sample for instance due to non-perfect fluid exchange in the measuring cell.

\subsubsection{Instrumentation and experimental procedures}

The measurements are performed using an Asylum ES AFM equipped with photo-thermal excitation (Blue drive), a sealed fluid cell and a temperature control unit. We use two types of AFM cantilevers, either with a colloidal probe or a small tip with a flattened contact surface. The cantilever of the colloidal probe is a rectangular beam with a cone-shaped silicon tip (Team nanotec, LRCH, $R=1080 \mathrm{~nm}$ ). The beam is a bi-layer of typically $1.5 \mu \mathrm{m}$ thick silicon with a $50 \mathrm{~nm}$ thick coating of gold. Length and width of the beam are about 150 and $15 \mu \mathrm{m}$, respectively. The total tip cone height is typically $15 \mu \mathrm{m}$ and the full-cone angle $45^{\circ}$. The cantilever of the small tip is a rectangular beam with dimensions $130 \times 35 \times 2 \mu \mathrm{m}^{3}$, coated with a $50 \mathrm{~nm}$ gold layer (Mikromasch, HQ:NSC35/Cr-Au BS, $R=8 \mathrm{~nm}$ ). The cone height is approximately $15 \mu \mathrm{m}$. To avoid variations of the tip curvature during the measurements, we flatten the cantilever tip after it is immersed in the liquid by imaging the substrate in contact mode with a constant load of about 50 $\mathrm{nN}$ over approximately 10 minutes. The resulting radius of the contact area is $40 \mathrm{~nm}$, as determined from a SEM image taken after the experiments were performed.

The AFM cantilever is completely immersed in a droplet of electrolyte that is sandwiched between the substrate and the top of the cell. The volume of the droplet is $0.2 \mathrm{~mL}$. The electrolyte is injected and removed via a pair of plastic syringes (free of lubricants). To exchange the electrolytes, a new solution is injected via the inlet while the mixed solution is sucked out via the outlet of the fluid cell. The exchange stops after injecting $4 \mathrm{~mL}$, which is assumed to be sufficient to remove all the original liquid. After exchange of electrolyte, we wait approximately $10 \mathrm{~min}$ to equilibrate the system. The AFM scanner is placed in a chamber with the temperature set to $30{ }^{\circ} \mathrm{C}$.

For the actual measurement of the tip-sample interactions, the cantilever is driven at a fixed frequency ( $\omega \approx 0.7 \omega_{0}$ for the colloidal probe, $\omega \approx 0.97 \omega_{0}$ for the small tip) by an intensity-modulated blue laser diode that is focused on the gold coated topside of the cantilever close to its base. This direct 

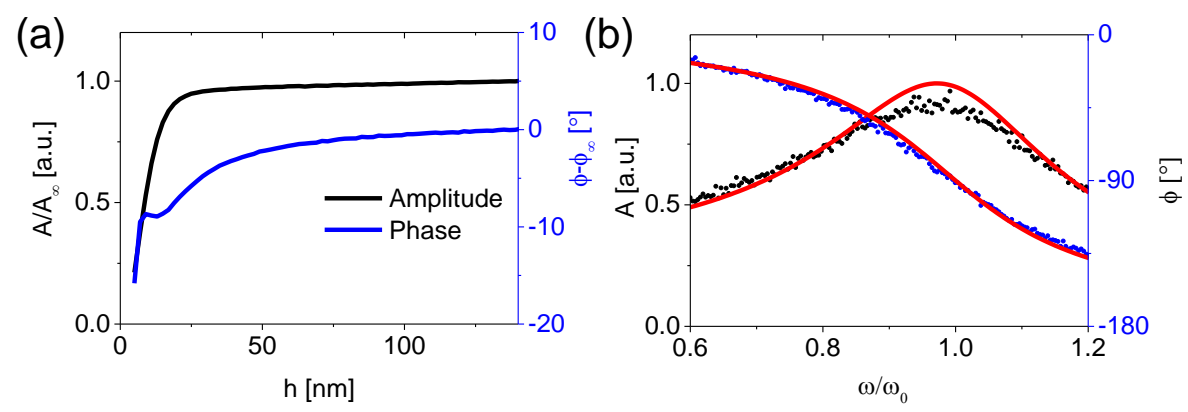

Figure 5.2: (a) Example of an amplitude- (black) and phase- (blue) distance curve, measured with a colloidal tip. The fluid is $\mathrm{pH} 5.7 / 100 \mathrm{mM} \mathrm{NaCl}$ solution. The amplitude is normalized by $A_{\infty}$, the phase $\phi$ is with respect to $\phi_{\infty}$, both measured at $140 \mathrm{~nm}$. (b) Measured frequency response of the same cantilever (amplitude: black dots, phase: blue dots) under photo-thermal excitation in liquid. The red lines represent the calculated response using the cantilever parameters determined from its thermal noise spectrum. Experimental parameters: $k_{c}=0.66 \mathrm{~N} / \mathrm{m}, Q=3, \omega_{0} / 2 \pi=16.11 \mathrm{kHz}, \omega / 2 \pi=11$ $\mathrm{kHz}$ and $A_{\infty} \approx 0.8 \mathrm{~nm}$.

photo-thermal driving prevents the excitation of extra resonances ('forest of peaks') usually observed with acoustic driving [46], that complicates the analysis severely, as described in the preceding chapter [10]. Figure 5.2 shows a very smooth transfer function which is characteristic for photo-thermal excitation. The amplitude of the cantilever oscillation is set to approximately $0.8 \mathrm{~nm}$ by tuning the intensity of the blue laser. For each amplitude- or phase-distance curve, the distance between cantilever and surface is ramped from 140 to $0 \mathrm{~nm}$ at a ramp velocity of $75 \mathrm{~nm} / \mathrm{s}$. For each fluid composition we measure typically 10 to 30 approach curves. Without photo-thermal excitation, the static deflection against the piezo displacement is captured for calibration of the tip-substrate separation (see the preceding chapter for more detail). It takes approximately 20 to 30 minutes to measure one fluid composition. Judging from the amplitude- and phase-distance curves, the tip-sample interaction does not vary within this timespan. This implies that the $\mathrm{pH}$ level stays constant and the solution does not suffer from adsorption of, for instance, $\mathrm{CO}_{2}$ and that the tip does not degrade.

Before exchanging the solution, the cantilever is withdrawn from the surface by approximately $1 \mu \mathrm{m}$, using the AFM piezo. The position of the 

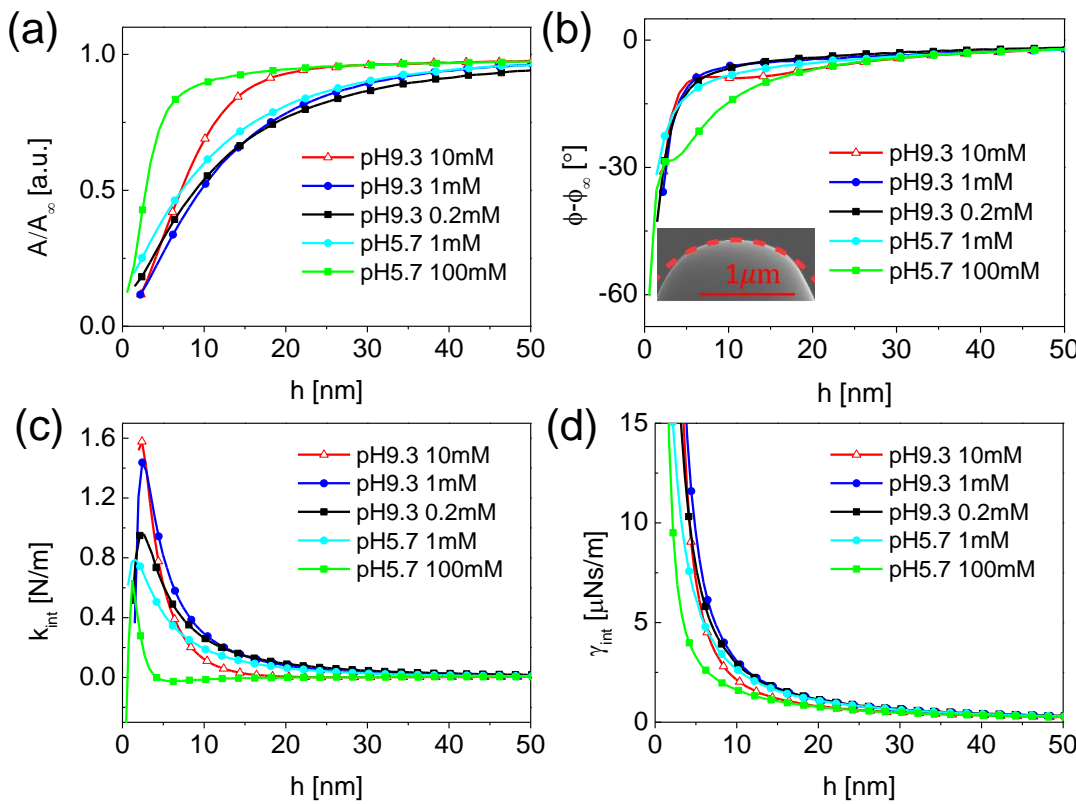

Figure 5.3: Amplitude (a) and phase (b) response of the colloidal probe cantilever as a function of tip-substrate separation. The amplitude is normalized by $A_{\infty}$, the phase is with respect to $\phi_{\infty}=0$. From these data the interaction stiffness $k_{\text {int }}(\mathrm{c})$ and interaction damping $\gamma_{\text {int }}(\mathrm{d})$ are determined.

driving laser and the detection laser is kept fixed with respect to the cantilever throughout all the measurements. In Fig. 5.3, we show the captured amplitude and phase response as a function of the tip-substrate separation.

\subsection{Results}

In this section we present our experimental results obtained with a colloidal probe and with a flattened tip, which has a much smaller interaction area. Although in detail quite different, the results obtained with the two tips show the same trends. Unless otherwise stated, the reported data are measured with a colloidal probe cantilever. 


\subsubsection{Conservative forces and surface charge}

In Fig. 5.3, the primary results of our measurements with the colloidal probe have been presented. The characteristic transition near a tip-substrate distance of $5 \mathrm{~nm}$, in the $A(h)$ and the $\phi(h)$ curves, as given in Fig. 5.3 (a) and (b), reflects the range of the electrostatic interactions which is given by the Debye length $\kappa^{-1}$. For a $1 \mathrm{mM}$ salt solution it is approximately $10 \mathrm{~nm}$. The resulting force gradient (interaction stiffness) in Fig. 5.3(c) shows this dependence on $\kappa^{-1}$ even better: the final exponential decays are 1/3.2, 1/10 and $1 / 18 \mathrm{~nm}^{-1}$ for 10,1 and $0.1 \mathrm{mM}(\mathrm{pH}=9.3)$, respectively, see also Fig. 5.4(d). For 10 and $1 \mathrm{mM}$ these values match with the estimated Debye lengths of 3 and $10 \mathrm{~nm}$. For the lowest concentration we conclude from this observation that the intended concentration of $0.1 \mathrm{mM}$ is in fact $0.2 \mathrm{mM}$. At these low concentrations the solutions are very sensitive to slight contamination of the sample for instance due to non-perfect fluid exchange in the measuring cell, which can explain the observed deviation. To relate the measured interaction stiffness with the electrostatic properties of the electrolyte film between tip and substrate, we consider DLVO theory and charge regulation.

\section{DLVO calculation}

In the DLVO force analysis, we follow the method presented in Ref. [9]. The conservative DLVO force $F$ between tip and substrate results from three contributions, i.e. the osmotic, electrostatic and van der Waals force:

$$
F=\int_{0}^{\infty}\left\{\Pi_{\mathrm{osm}}(h)+\Pi_{\mathrm{el}}(h)+\Pi_{v d W}(h)\right\} 2 \pi r d r
$$

where $h=h_{0}+r^{2} /\left(2 R_{\mathrm{tip}}\right)$ represents the tip-substrate distance $h_{0}$ and the shape of the tip $r^{2} /\left(2 R_{\text {tip }}\right)$. Hence, the corresponding force gradient or interaction stiffness $k_{\mathrm{int}}=-\partial F_{\mathrm{int}} / \partial h_{0}$ will be given by:

$$
k_{\text {int }}(h)=2 \pi R_{\text {tip }}\left\{\Pi_{\mathrm{osm}}(h)+\Pi_{\mathrm{el}}(h)+\Pi_{v d W}(h)\right\}
$$

The first term within the braces represents the osmotic contribution:

$$
\Pi_{\mathrm{osm}}(h)=k_{B} T \sum_{i} n_{i}^{\infty}\left(e^{-\frac{Z_{i} e \phi}{k_{B} T}}-1\right)
$$

with $\phi$ the electric potential, $e$ the elementary charge and $k_{B} T$ the thermal energy. $Z_{i}$ is the valency and $n_{i}^{\infty}$ the bulk concentration of ions of species $i$. In our experiment four ionic species are present, as there are cations and 
anions from dissolved $\mathrm{NaCl}$ as well as hydroxide and hydrogen ions due to auto-hydrolysis. The second term represents the electrostatic contribution:

$$
\Pi_{\mathrm{el}}(h)=-\frac{1}{2} \varepsilon_{0} \varepsilon_{r}\left(\frac{\partial \phi}{\partial z}\right)^{2}
$$

with $\varepsilon_{0}$ the electric permittivity of vacuum and $\varepsilon_{r}$ the relative dielectric constant of water. The third term represents the van der Waals forces:

$$
\Pi_{\mathrm{vdW}}(h)=-\frac{A_{H}}{6 \pi h^{3}}
$$

$A_{H}$ represents the Hamaker constant. Note that $\Pi_{\mathrm{osm}}$ and $\Pi_{\mathrm{el}}$ depend on both the tip-substrate distance $h$ and the coordinate $z$, measured perpendicular to the surfaces, but their sum turns out to depend only on $h$. Calculation of the osmotic and electrostatic contribution requires the solution of the electric potential between the tip and the substrate. This potential is governed by the Poisson-Boltzmann equation:

$$
\varepsilon_{0} \varepsilon_{r} \frac{\partial^{2} \phi}{\partial z^{2}}=-\sum_{i} e Z_{i} n_{i}^{\infty} e^{-\frac{Z_{i} e \phi}{k_{B} T}}
$$

Last equation is conventionally solved by assuming either constant charge or constant potential on tip and substrate. These assumptions are justified for large tip-substrate separations (where tip and substrate only weakly interact with each other), but usually fail in the regime of small tip-substrate distances [9]. In this regime $\left(\kappa h<10\right.$, where $\kappa=\left(e^{2} \sum Z_{i}^{2} n_{i}^{\infty} / \varepsilon_{0} \varepsilon_{r} k_{B} T\right)^{1 / 2}$ is the reciprocal Debye length) the local charge density and potential vary with separation distance $h$ to compensate the confinement-induced modification of the surface chemistry. We consider two surface reactions, deprotonation of the silanol groups and adsorption of $\mathrm{Na}^{+}$on the deprotonated sites [9]:

$$
\begin{aligned}
& \vdash \mathrm{SiOH} \rightleftharpoons \vdash \mathrm{SiO}^{-}+\mathrm{H}^{+} \\
& \vdash \mathrm{SiONa} \rightleftharpoons \vdash \mathrm{SiO}^{-}+\mathrm{Na}^{+}
\end{aligned}
$$

with equilibrium constants

$$
K_{\mathrm{H}}=10^{-p K_{\mathrm{H}}}=\frac{\left\{\mathrm{SiO}^{-}\right\}\left[\mathrm{H}^{+}\right]_{s}}{\{\mathrm{SiOH}\}}
$$

and

$$
K_{\mathrm{Na}}=10^{-p K_{\mathrm{Na}}}=\frac{\left\{\mathrm{SiO}^{-}\right\}\left[\mathrm{Na}^{+}\right]_{d}}{\{\mathrm{SiONa}\}}
$$


respectively. Here the index $s$ indicates the surface itself and $d$ the boundary between the Stern layer and the diffuse part of the double layer. The total site density is conserved:

$$
\begin{aligned}
\Gamma & =\{\mathrm{SiOH}\}+\{\mathrm{SiONa}\}+\left\{\mathrm{SiO}^{-}\right\} \\
& =\left\{\mathrm{SiO}^{-}\right\}\left(1+10^{p K_{\mathrm{H}}}\left[\mathrm{H}^{+}\right]_{s}+10^{p K_{\mathrm{Na}}}\left[\mathrm{Na}^{+}\right]_{d}\right)
\end{aligned}
$$

Note that the distribution of $\mathrm{Na}^{+}$and $\mathrm{H}^{+}$ions is governed by the Boltzmann relation, and their concentrations near the tip and substrate differ from the bulk. The surface potential $\phi_{s}$ is related to the diffuse layer potential $\phi_{d}$ via the Stern layer capacitance: $C_{s}=\varepsilon \varepsilon_{0} / d_{s}=\sigma /\left(\phi_{s}-\phi_{d}\right)$. Combining Eqs. (5.15) to (5.17) the surface charge $\sigma=-e\left\{\mathrm{SiO}^{-}\right\}$can be expressed as:

$$
\sigma\left(\phi_{s}\right)=\frac{-e \Gamma}{1+\left(\frac{\left[H^{+}\right]_{\infty}}{K_{\mathrm{H}}}+e^{\frac{e \sigma d_{s}}{\varepsilon \varepsilon_{0} k_{B} T}} \frac{\left[N a^{+}\right]_{\infty}}{K_{\mathrm{Na}}}\right) e^{-\frac{e \phi_{s}}{k_{B} T}}}
$$

Note that the surface charge is essentially 0-plane charge plus Stern layer, i.e. equal to diffuse layer charge in magnitude.

\section{Extracting the surface charge}

Equation (5.18) provides the boundary conditions for Eq. (5.13). The bulk concentration of the ions is known. The site density and the stern layer capacitance are more consistently reported in literature than the $p K$ values of the considered reactions [9]. Therefore, we take $p K_{\mathrm{H}}$ and $p K_{\mathrm{Na}}$ as the parameters to fit. For each pair of $p K$ values we can evaluate $k_{\text {int }}^{\text {calc numerically }}$ as a function of tip-substrate distance $h$ and compare them with the experimentally found $k_{\text {int }}^{\text {exp }}$. The fit quality is characterized by a merit function $1 / \chi^{2}=1 / \sum_{j}\left[k_{\text {int }}^{\text {calc }}(j)-k_{\text {int }}^{\exp }(j)\right]^{2}[9]$. During the fitting, data at separations $h<5 \mathrm{~nm}$ are disregarded, because the DLVO theory as such will work down to smaller separations than $5 \mathrm{~nm}$, but the geometry in the colloidal probe case is not that well known. In Fig. 5.4(a), we show the merit function for the data measured at $\mathrm{pH} 9.3 / 1 \mathrm{mM}$. The function is normalized by its maximum. It is worthwhile to note that the best fitting pairs are not unique. This is because the force gradient $k_{\text {int }}$ is governed by $\sigma$, which is a function of both $p K_{\mathrm{H}}$ and $p K_{\mathrm{Na}}$, as shown in Eq. (5.18). The decrease of charge by promoting deprotonation, e.g. decreasing the $p K_{\mathrm{H}}$, can be fully compensated by promoting at the same time cation adsorption, e.g. increasing the $p K_{\mathrm{Na}}$. This explains the strong correlation between the optimal values of $p K_{\mathrm{H}}$ and 

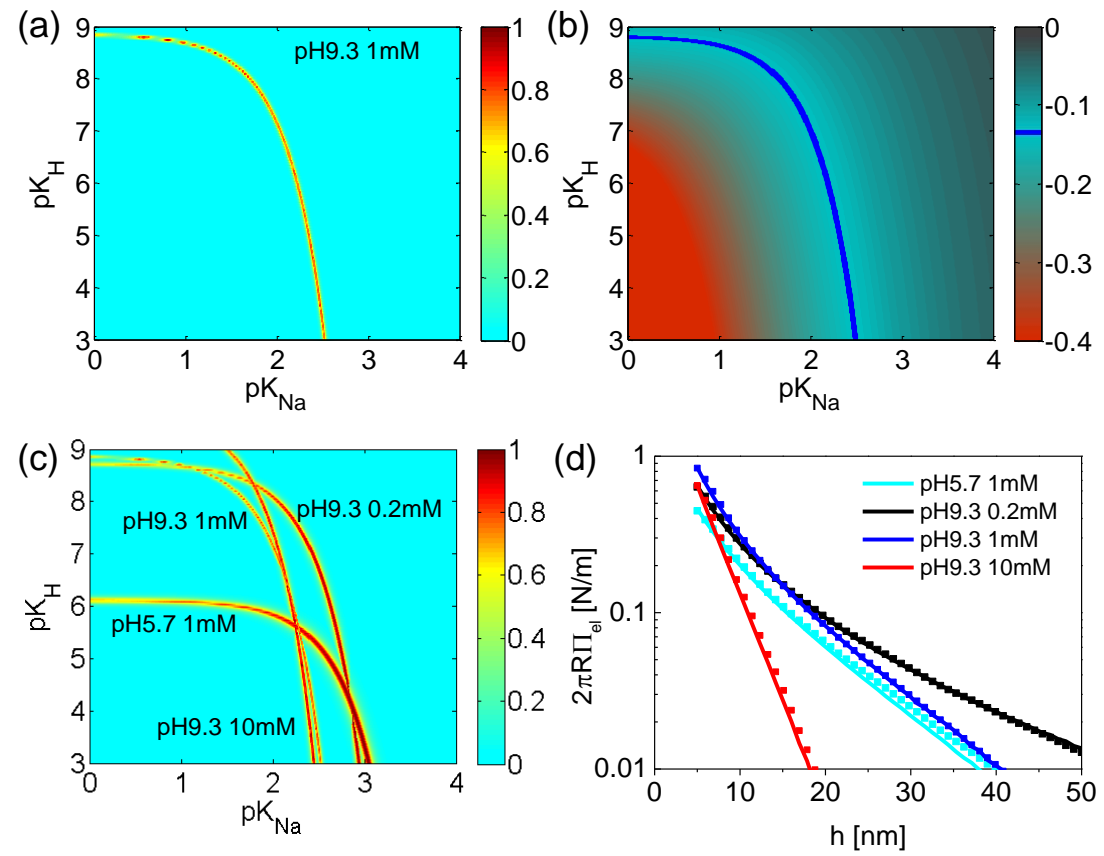

Figure 5.4: (a) The normalized merit function for the tested $\left(p K_{H}, p K_{N a}\right)$ pairs for a solution with $\mathrm{pH} 9.3 / 1 \mathrm{mM}$. (b) The contour plot of the surface charge; the blue line matches the maximum merit curve in (a). (c) The normalized merit function for the tested $\left(p K_{H}, p K_{N a}\right)$ pairs for all four solutions. (d) The electrostatic part of the measured interaction stiffness versus tip-substrate distance (dots) and the best fitting calculated curves (lines) for the four solutions: $\mathrm{pH} 5.7 / 1 \mathrm{mM}$ (light blue), $\mathrm{pH} 9.3 / 0.2 \mathrm{mM}$ (black), $\mathrm{pH}$ 9.3/1 mM (blue) and $\mathrm{pH} 9.3 / 10 \mathrm{mM}$ (red). 


\begin{tabular}{lllllll} 
& & \multicolumn{2}{c}{ colloidal tip } & \multicolumn{2}{l}{ small tip } \\
\hline $\mathrm{pH}$ & {$[\mathrm{NaCl}] / \mathrm{mM}$ (aimed) } & $p K_{H}$ & $p K_{N a}$ & $p K_{H}$ & $p K_{N a}$ \\
\hline \multirow{4}{*}{5.7} & 1 & $(1)$ & 6.00 & 1.75 & - & - \\
& 4 & $(1)$ & - & - & 7.22 & 2.47 \\
& 20 & $(10)$ & - & - & 7.22 & 2.31 \\
& 100 & $(100)$ & - & - & - & - \\
\hline \multirow{4}{*}{9.3} & 0.2 & $(0.1)$ & 8.35 & 1.78 & - & - \\
& 2 & $(1)$ & 7.84 & 1.78 & 8.99 & 1.69 \\
& 10 & $(10)$ & 8.35 & 1.78 & - & - \\
& 20 & $(10)$ & - & - & 8.61 & 1.78 \\
& 100 & $(100)$ & - & - & 8.86 & 1.61
\end{tabular}

Table 5.1: The best fitting pK values obtained for the several electrolyte solutions. Note: The aimed $[\mathrm{NaCl}]$ concentrations are given in brackets.

$p K_{\mathrm{Na}}$ as observed in Fig. 5.4(a). Ideally, we should get a smooth continuous optimal merit curve. However, because the spacing between the tested pair values is finite, a slight variation along the optimal merit curve is observed. This variation can be suppressed by decreasing this spacing, at the expense of considerably longer calculation times. In Fig. 5.4(b) the merit functions for all relevant electrolyte solutions have been shown. Because the strength of the compensation mechanism just described, is different for every solution, we expect different optimal merit curves. However, provided that the equilibrium constants are invariant under $\mathrm{pH}$ or ionic strength variation (and that the experimental errors are negligible), we expect one unique $\left(p K_{\mathrm{Na}}, p K_{\mathrm{H}}\right)$ pair for all conditions, and all the curves should cross this unique point. As we observe from Fig. 5.4(b) the maximum merit curves for $\mathrm{pH}=9.3$ indeed cross each other almost in a single point near $\left(p K_{\mathrm{Na}}, p K_{\mathrm{H}}\right)=(1.7 \pm 0.1,8.2 \pm 0.4)$. But the curve for $\mathrm{pH}=5.7$ strongly deviates. So, we get a quasi-triangular region near $\left(p K_{\mathrm{Na}}, p K_{\mathrm{H}}\right)=(2.0 \pm 0.4,7 \pm 1.4)$. These values are in agreement with previous findings $[9,50,51]$. As the value of this optimum is considerably lower than the optimum for only $\mathrm{pH}=9.3$, we conclude that we can not describe the charge regulation with the simple surface complexation model used here. Given the complexity of the EDL as shown in Ref. [12], it is not surprising that the structure may actually be more complex than a single pK pair suggests. However, on the level of surface charge determination the approach is quite successful. As can be seen from Fig. 5.4(b), the individual 
(a)

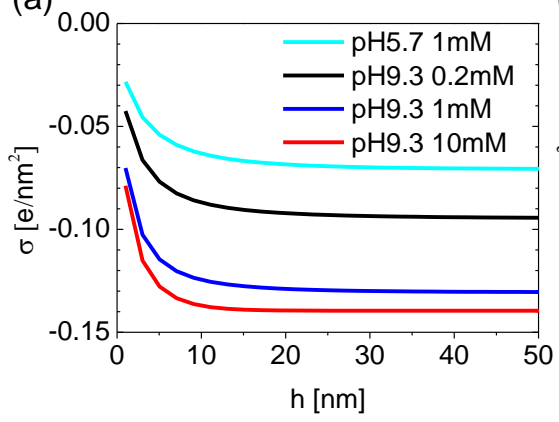

(b)

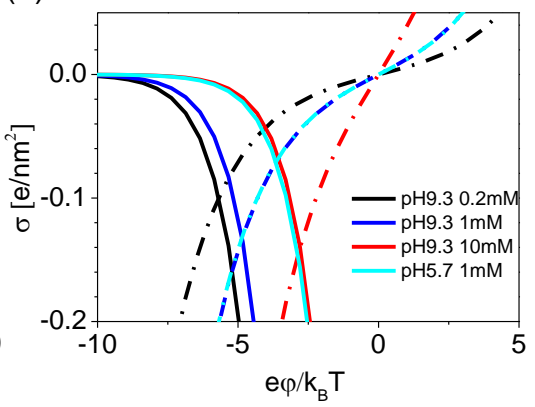

Figure 5.5: (a) The calculated surface charge as a function of separation distance. The color scheme is the same as in Fig. 5.3. (b) The surface charge versus dimensionless potential as obtained from charge regulation (full lines) and from the PB equation (Grahame equation, dotted lines), for the four solutions. The crossing of corresponding curves indicate the actual surface charge at large tip-substrate separations.

optimal merit curves nicely coincides with the curves for constant charge.

In Fig. 5.4(d), the best fitting interaction stiffness curves are compared with the experimental data, showing in all cases a nice agreement between fit and experiment. The resulting optimal $p K$ values for the individual curves have been listed in Table 5.1. In Fig. 5.5(a), the resulting surface charge is shown as a function of the tip-surface separation. The magnitude of the surface charge decreases with decreasing separation. This is a consequence of the charge regulation: the concentration of both $\mathrm{H}^{+}$and $\mathrm{Na}^{+}$increases in the overlapping diffuse layer when it becomes thinner. Therefore, the chemical equilibrium shifts towards a higher $\mathrm{H}^{+}$and $\mathrm{Na}^{+}$adsorption, reducing the number of charged sites on the substrates. As expected, the surface charge dramatically increases when the $\mathrm{pH}$ changes from 5.7 to 9.3. At high $\mathrm{pH}$, deprotonation of the silica substrate is favored which results in a more negative surface. At a $\mathrm{Na}^{+}$concentration of $1 \mathrm{mM}$, the surface charge increases from -0.07 to $-0.13 \mathrm{e} / \mathrm{nm}^{2}$. The concentration dependence as observed in Fig. 5.5(b) is less obvious. On the one hand due to the increasing bulk concentration of the sodium, $\left[\mathrm{Na}^{+}\right]_{\infty}$, the equilibrium should shift towards a higher sodium adsorption. This is represented by the shift of the charge regulation curves (Eq. (5.18), the full lines in Fig. 5.5) to the right with increasing concentration. But on the other hand the Debye length (which is 
hardly affected by $p H$ variation) decreases. This steepens the solution of the Poisson Boltzmann equation (Eq. (5.13), the dotted lines in Fig. 5.5) with increasing concentration. This means the adsorption is reduced. The net effect is a gradual increase of the surface charge with increasing concentration as can be observed from the downward shift in the crossing points.

The corresponding data for a small tip cantilever are presented in Fig. 5.6. Panel (a) shows the measured interaction stiffness (only electrostatic part) compared with the best fitting calculated curves. Again, the agreement is satisfactory.

In the fitting procedure, the data for $h<3 \mathrm{~nm}$ are excluded from the analysis to avoid the influence of hydration forces. The concentrations are corrected, as shown in Table 5.1, using the decaying length of the electrostatic force gradient $k_{e l}$. Due to lack of data points, the data for $\mathrm{pH} 5.7 / 100$ $\mathrm{mM}$ have not been analyzed. For the other fluid compositions, the resulting surface charge as a function of tip-substrate distance is given in panel (c). The same trends are observed as discussed for the colloidal probe in Fig. 5.5. From the merit curves (not shown), we observe qualitatively the same behavior as for the colloidal probe: For $\mathrm{pH}=9.3$, the maximum merit curves cross each other near a single point $\left(p K_{\mathrm{Na}}, p K_{\mathrm{H}}\right)=(1.7 \pm 0.1,8.8 \pm 0.2)$ while the curves for $\mathrm{pH}=5.7$ meet each other near $\left(p K_{\mathrm{Na}}, p K_{\mathrm{H}}\right)=(2.4 \pm 0.1,7.3 \pm 0.2)$. All $\mathrm{pK}$ values have been collected in Table 5.1. The results for the colloidal probe differ slightly from those for the small tip, but the deviations are close to the uncertainties of the $\mathrm{pK}$ values.

\subsubsection{Dissipation and ionic charge distribution}

In Fig. 5.3(d), the measured viscous dissipation coefficients, $\gamma_{\text {int }}$, have been plotted as a function of the tip-substrate distance, $h$. Again, a clear dependence on ionic strength and $\mathrm{pH}$ is visible. For reference, we calculate the hydrodynamic dissipation for a spherical tip approaching a flat substrate. Assuming no slip on the tip or the substrate, the hydrodynamic dissipation coefficient $\gamma_{\text {ref }}$ is (for $h_{0} / R_{\text {tip }} \ll 1$ ) given by:

$$
\gamma_{\mathrm{ref}}\left(h_{0}\right)=\eta_{b} \frac{6 \pi R_{\mathrm{tip}}^{2}}{h_{0}}
$$

where $\eta_{b}$ is the bulk viscosity of the solution, $h_{0}$ the separation between the tip apex and the substrate. The viscosity of water at $30^{\circ} \mathrm{C}$ is $0.8 \mathrm{mPa} \cdot \mathrm{s}$. We expect that the measured dissipation for the $\mathrm{pH} 5.7 / 100 \mathrm{mM}$ solution behaves for $h>10 \mathrm{~nm}$ like the reference because, for this curve, the surface 
(a)
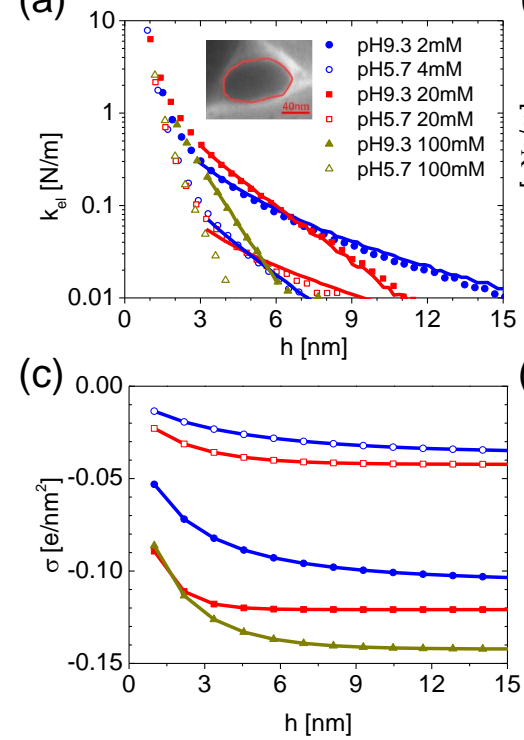

(b)

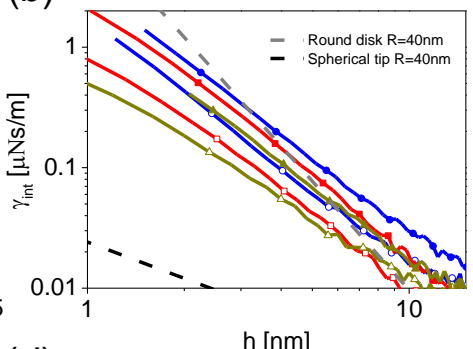

(d)

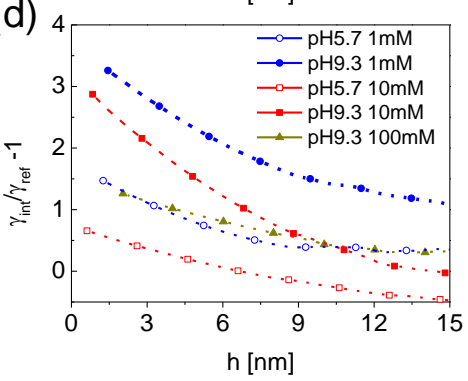

Figure 5.6: Results obtained with a small flattened tip. (a) The measured electrostatic force gradient and the fit with the DLVO theory. The data at $h<3 \mathrm{~nm}$ were excluded from the fit. The inset is an SEM image of the tip after use. The contour of the flat part is highlighted in red. (b) The measured damping coefficient. The dash lines represent the calculated dissipation, assuming no electro-viscous enhancement, for a round disk of $R=40 \mathrm{~nm}$ (gray line) and for a spherical tip with $R=40 \mathrm{~nm}$ (black line). (c) Extracted surface charge density as a function of tip-substrate separation. (d) The damping enhancement with respect to the reference case, $\mathrm{pH} 5.7 / 100$ mM. Experimental parameters: $k_{c}=5.46 \mathrm{~N} / \mathrm{m}, Q=5.3, \omega_{0} / 2 \pi=62.75 \mathrm{kHz}$, $\omega / 2 \pi=61.20 \mathrm{kHz}$ and $A_{\infty} \approx 0.9 \mathrm{~nm}$. 

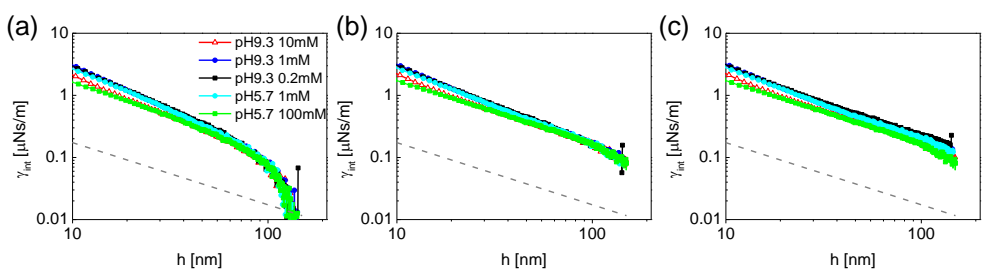

Figure 5.7: Log-log plots of the dissipation coefficient, $\gamma_{\text {int }}$, measured in $\mathrm{nNs} / \mathrm{m}$, versus tip-substrate distance $h$. The reference case, $\mathrm{pH} 5.7 / 100 \mathrm{mM}$, has been plotted in green. Other curves are: $\mathrm{pH} 9.3 / 0.2 \mathrm{mM}$ (black), $\mathrm{pH} 9.3 / 1$ $\mathrm{mM}$ (blue), pH 9.3/10 mM (red) and pH 5.7/1 mM (light blue). The dashed line has a slope -1. (a) Uncorrected dissipation coefficient, $\gamma_{\text {int }}^{\text {(unc) }}$ versus $h$. (b) Corrected $\gamma_{\text {int }}$ versus $h$. The correction for the damping coefficient at $h$ $=140 \mathrm{~nm}$ is taken as $\gamma_{\text {offset }}=90 \mathrm{nNs} / \mathrm{m}$. (c) Corrected $\gamma_{\text {int }}$ versus $h$. Now $\gamma_{\text {offset }}$ is considered variable for the different fluid compositions.

charge is fully screened within the first $10 \mathrm{~nm}$ due to the short Debye length of $3 \mathrm{~nm}$.

Before we compare the calculated dissipation with the measured one, we evaluate the effect of the calibration procedure on the measured damping coefficient. We determined the value for $\gamma_{c}$ by assuming that at $140 \mathrm{~nm}$ the interaction with the substrate is negligible. However, for a colloidal probe with a radius of $1.08 \mu \mathrm{m}$ the hydrodynamic damping is approximately 90 $\mathrm{nNs} / \mathrm{m}$ as we learn from Eq. (5.19), while we obtained from our callibration $\gamma_{c} \approx 2.17 \mu \mathrm{Ns} / \mathrm{m}$. The uncorrected dissipation coefficients in Fig. 5.7(a) deviate strongly from the expected $h^{-1}$ behavior for $h>100 \mathrm{~nm}$ and asymptotes towards zero near $h=140 \mathrm{~nm}(\log h=2.146)$. If we correct for this effect by adding the estimated $\gamma_{\text {offset }}=90 \mathrm{nNs} / \mathrm{m}$ to all measured dissipation values, the resulting curves in Fig. 5.7(b) show now approximately the expected $h^{-1}$ asymptotic behavior, but have all the same value at $h=140 \mathrm{~nm}$. If we optimize every single curve for a $h^{-1}$ asymptotic behavior, we obtain the curves in Fig. 5.7(c). However, except for the reference case, we do not know the exact limiting slope of the dissipation curves, because we do not know the limiting behavior of the enhancement. In fact, for the correction in Fig. 5.7(c), we assume that the enhancement is negligible for say $h>80 \mathrm{~nm}$. So we may assume that panel b and c of Fig. 5.7 show the lower and upper bound for the real dissipation behavior. To get a better impression of this uncertainty we plot in Fig. 5.8 the reciprocal dissipation coefficient versus the 
(a)

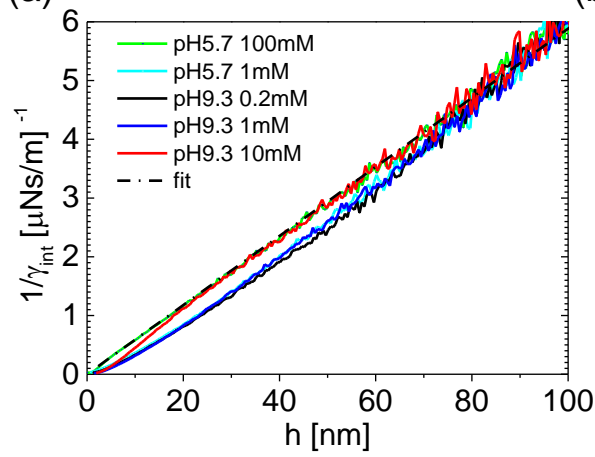

(b)

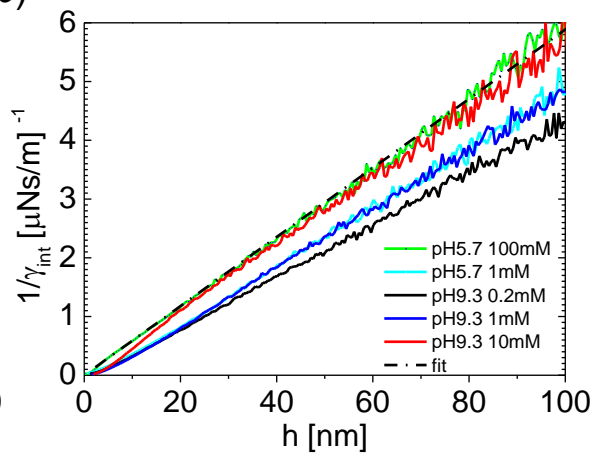

Figure 5.8: (a) The measured reciprocal dissipation coefficient versus tipsubstrate distance. The reference case, $\mathrm{pH}$ 5.7/100 mM, has been plotted in green. The dashed line represents the calculated Reynolds damping, using Eq. 5.19. The damping coefficient at the tail is taken as the same, $\gamma_{\text {int }}$ $(\mathrm{h}=140 \mathrm{~nm})=90 \mathrm{nNs} / \mathrm{m}$. (b) The reciprocal dissipation coefficient versus tip-substrate distance. The damping coefficient at $\mathrm{h}=140 \mathrm{~nm}$ is considered as a variable for different fluid compositions.

tip-substrate distance $h$ for these two cases. For $h>50 \mathrm{~nm}$ the deviation is clear, but for $h<30 \mathrm{~nm}$ the corresponding curves in both panels are identical and we consider them as reliable. Therefore, we analyze the dissipation data only for $3<h<30 \mathrm{~nm}$.

The calculated Reynolds dissipation, Eq. (5.19), has been fitted to the corrected damping coefficients, measured for $\mathrm{pH}$ 5.7/100 mM, see Fig. 5.9. The fitted value for the viscosity is $0.77 \mathrm{mPa} \cdot \mathrm{s}$, which is in excellent agreement with the bulk viscosity of the solution, i.e. $0.8 \mathrm{mPa} \cdot \mathrm{s}$ at $30^{\circ} \mathrm{C}$. It also confirms the no slip assumption, which is in agreement with previous findings [52].

In contrast to the data measured at $\mathrm{pH} 5.7 / 100 \mathrm{mM}$, the dissipation, $\gamma_{\text {int }}(h)$, measured for the other four electrolyte solutions significantly deviates from the Reynolds dissipation. This implies that the dissipation, and so the friction, in the electrolyte film depends on the ionic charge distribution. We take $\gamma_{\text {int }}(h)$ measured for the solution with $\mathrm{pH} 5.7 / 100 \mathrm{mM}$ as the "zero charge" reference $\gamma_{\mathrm{ref}}(h)$ and calculate the enhancement of the dissipation which we define as $c_{e v}=\gamma_{\text {int }}(h) / \gamma_{\text {ref }}(h)-1$. In Fig. 5.10(a) the enhancement 
(a)

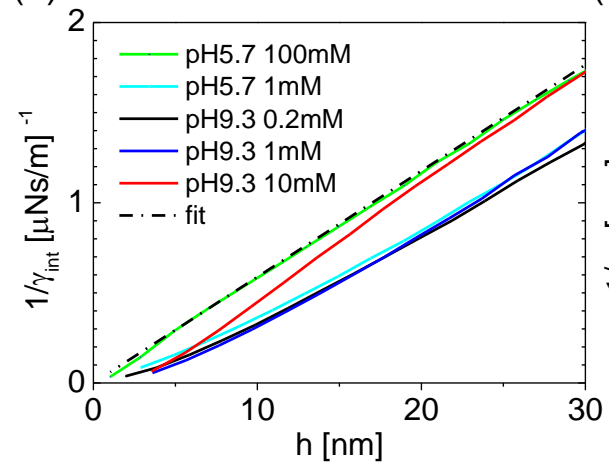

(b)

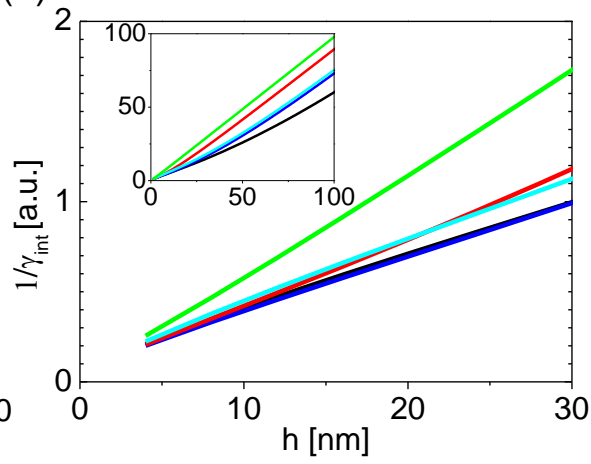

Figure 5.9: The measured (a) and calculated (b) reciprocal dissipation coefficients versus tip-substrate distance. The dotted line in (a) represents the Reynolds fit. The curves in (b) have been calculated with Eq. 5.25. Color scheme as before.

$c_{e v}$ versus $h$ has been shown for several electrolyte solutions. When the tip approaches the substrate (decreasing separation), the enhancement increases monotonically until it reaches a maximum around $h \approx 3 \mathrm{~nm}$. On further approach the enhancement decreases again, but then the tip is so close to the interface that non-electric interactions like van der Waals forces and solvation forces come into play. Therefore we do not consider these short distances in our analysis.

In Fig. 5.6 (b) and (c) we present the damping data collected with the small flattened tip. Panel (b) shows a log-log plot of $\gamma_{\text {int }}$ versus $h$. The overall slope is rather close to $h^{-2}$, which is in between $h^{-1}$, as expected for a spherical tip, and $h^{-3}$ as expected for a disk-like tip. Because the interaction area is much smaller than that of a colloidal tip, the data for $h>10 \mathrm{~nm}$ are dominated by noise, which limits the distance range of our analysis. Because the surface charge for the $\mathrm{pH} 5.7 / 100 \mathrm{mM}$ solution is very low, $|\sigma|<0.01 \mathrm{e} / \mathrm{nm}^{2}$, we use this solution as a reference to calculate the damping enhancement, which is given in panel (d). To understand the dissipation behavior, we consider two effects, which are known in literature as the electro-viscous effect [22] and the visco-electric effect [34]. 
(a)

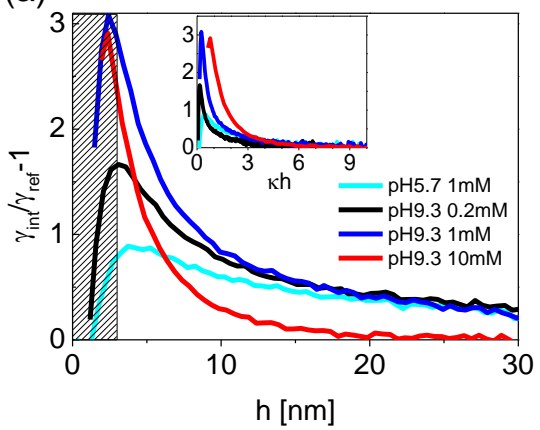

(b)

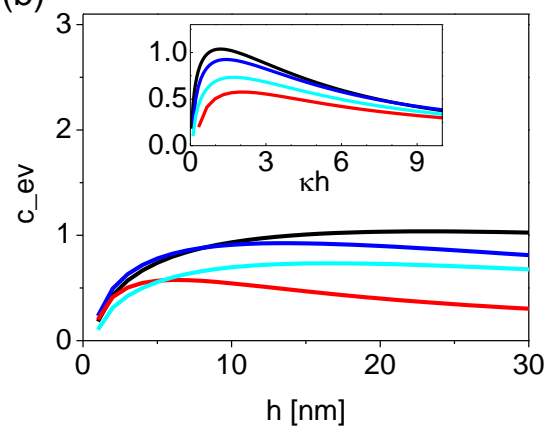

Figure 5.10: The measured (a) and calculated (b) $c_{e v}$ coefficients as a function of tip-substrate distance. Color scheme as before.

\section{Electro-viscous effect}

Due to the charge density on the substrate and tip surface, counter ions accumulate in the electrolyte film between the tip and the substrate, where the ions are trapped in the local potential field. This causes an additional body force on the electrolyte film that hinders the squeeze-out of liquid in the thin film when the tip approaches the substrate. This enhances the dissipation. To estimate this enhancement more quantitatively we assume that the ion distribution relaxes fast to its equilibrium distribution at the momentary tip-substrate distance on a timescale much shorter than the squeeze-out timescale. Knowing the mobility of the excess ions and their distribution in the film, we can calculate the resulting body force on the liquid and take this force into account when we calculate the pressure needed to squeeze-out the liquid from the film. The governing equations for the flow field $\underline{u}$ are given by:

$$
\nabla \cdot \underline{u}=0
$$

and:

$$
\rho\left(\frac{\partial \underline{\mathrm{u}}}{\partial t}+\underline{\mathrm{u}} \cdot \nabla \underline{\mathrm{u}}\right)=-\nabla p+\eta \nabla^{2} \underline{\mathrm{u}}+\underline{\mathrm{f}}_{b}
$$

Here $\rho$ is the density of the electrolyte, $\eta$ its viscosity and $\underline{\mathbf{f}}_{b}$ is the effective body force acting on the liquid. Because the equilibration time of the ions is short compared to the characteristic time of the oscillating flow, $\omega h^{2} / D_{\text {ion }} \simeq$ $5 \times 10^{-3}$ for $\omega / 2 \pi=10 \mathrm{kHz}, h=10 \mathrm{~nm}$ and $D_{\text {ion }} \simeq 10^{-9} \mathrm{~m}^{2} / \mathrm{s}$, we assume the equilibrium ion distributions, in stead of solving the Nernst-Planck equation 
for the ion densities and fluxes. Therefore, we model the body force, in radial direction,

$$
f_{b}=-\left|n^{+}-n^{-}\right| \frac{k_{B} T}{D_{\text {ion }}} u_{r}
$$

where $n^{+}$and $n^{-}$are the local cation and anion concentration, while $D_{\text {ion }}$ is the ion diffusion coefficient, in this case determined by the cation because both surfaces are negatively charged. Moreover, because $h^{2} \rho / \mu t_{0} \simeq 5 \times 10^{-6}$, $h \rho U_{0} / \mu \simeq 10^{-6}$ and $(h / R)^{2} \leq 4 \times 10^{-2}$ Eq. (5.20) and (5.21) reduce to:

$$
\begin{aligned}
\frac{1}{r} \partial_{r}\left(r u_{r}\right)+\partial_{z} u_{z} & =0 \\
\partial_{r} p & =\eta \partial_{z}^{2} u_{r}-\left|\sinh \left(e \phi / k_{B} T\right)\right| \frac{2 n_{\infty} k_{B} T}{D_{\text {ion }}} u_{r}
\end{aligned}
$$

Because $\phi(r, z)$ is known from our DLVO analysis, and $\partial_{r} p$ does not depend on $z$, we can solve $u_{r}(z)$ for given $r$ from

$$
\partial_{z}^{2} u_{r}=\frac{\partial_{r} p}{\eta}+\left|\sinh \left(e \phi / k_{B} T\right)\right| \frac{2 n_{\infty} k_{B} T}{\eta D_{\text {ion }}} u_{r}
$$

with the boundary conditions $u_{r}(0)=u_{r}(h)=0$ to get the flow profile. We mention that the Stern layer is assumed to be immobile and is not to yield under stress. Hence, separation $\mathrm{h}=0$ is reached when the Stern layers touch each other. From the flow profile, $u_{r}(z)$, we calculate $\left\langle u_{r}\right\rangle=-r U_{0} / 2 h$, to establish a relation between $\partial_{r} p$ and $U_{0}$. Here $U_{0}$ is the speed of the cantilever tip with respect to the substrate. The dissipative force is eventually calculated from the $\partial_{r} p$ profile under the tip:

$$
F_{\text {diss }}=\pi \int_{0}^{\infty} r^{2} \partial_{r} p d r
$$

Eventually, we obtain for a spherical tip and a flat substrate:

$$
\gamma_{\text {int }}=\frac{F_{\text {diss }}}{U_{0}}=\pi \eta R_{\mathrm{tip}}^{2} \int_{h_{0}}^{\infty} \frac{\left(h-h_{0}\right) d h}{h^{3} I(h)}
$$

where $I(h)=\left(h U_{0}\right)^{-1} \int_{0}^{h} u_{r}(z, h) d z$, depends on the double layer properties, see Eq. 5.23. In Appendix A we explain in detail how the actual calculations are performed. The results of this calculation will be presented and discussed in Section 5.4 . 


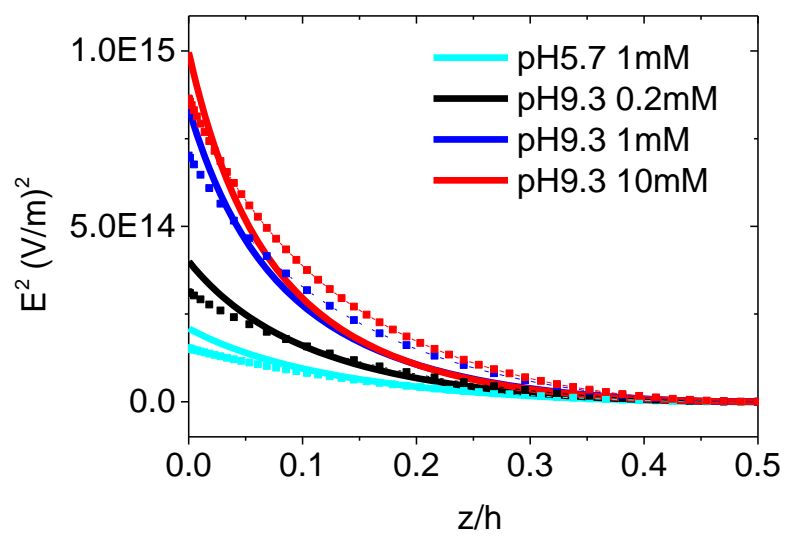

Figure 5.11: The electric field strength in the film between substrate and tip for $h=10 \mathrm{~nm}$ (lines) and $h=5 \mathrm{~nm}$ (dots) for $\mathrm{pH} \mathrm{9.3/0.2} \mathrm{mM} \mathrm{(black),} \mathrm{pH}$ 9.3/1 mM (blue), pH 9.3/10 mM (red) and pH 5.7/1 mM (light blue).

\section{Visco-electric effect}

In this section, we consider the electro-viscous effect, i.e. the effect of the viscosity increment due to the local electric field [34]. This increment is proportional to the electric field strength: $\eta=\eta_{0}\left(1+f_{v e} E^{2}\right)$, where $\eta_{0}$ is the zero field viscosity. The coefficient $f_{v e}$ has never been conclusively measured for water but it is estimated to be approximately $10^{-15}(\mathrm{~V} / \mathrm{m})^{-2}[36]$. To evaluate the significance of the visco-electric effect we need to know field strength in the liquid film. Just for an order of magnitude estimate, we simply approximate the tip-surface geometry as two parallel plates and neglect the electro-viscous effect in this case. When we solved the PB equation with charge regulation boundary conditions, we obtained the field strength in the diffuse layer for all separations and all fluid compositions.

In Fig. 5.11, the electric field in the gap is shown for the cases of $h=$ 5 and $h=10 \mathrm{~nm}$. Close to the wall, the electric field is in the order of $10^{7} \mathrm{~V} / \mathrm{m}$. In the middle of the gap, the field strength decays to zero due to the symmetry of the equally charged parallel plate geometry. To get an upper bound estimation, we choose for $f_{v e}$ three times the literature estimate, $f_{v e}=$ $3 \times 10^{-15}(\mathrm{~V} / \mathrm{m})^{-2}$, when we calculate the $\eta(z, h)$ dependence. With this dependence we recalculate the pressure gradient and finally the dissipation enhancement in the film for the case of a parabolic tip, see Appendix B for 
(a)

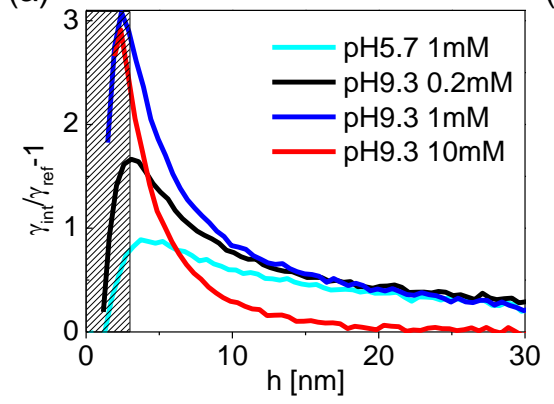

(b)

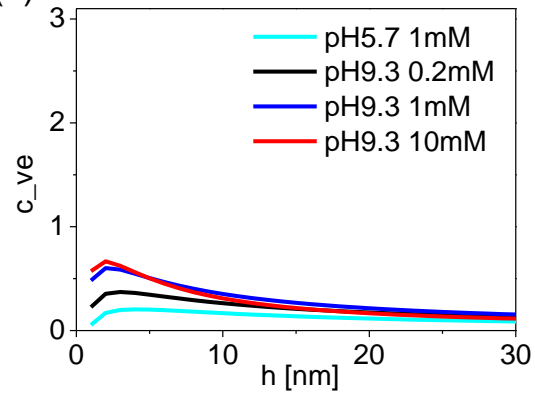

Figure 5.12: The measured (a) and calculated (b) viscosity enhancement coefficients, $c_{v e}$, as a function of tip-substrate distance. Color scheme as before.

more details.

The resulting enhancement of the dissipation has been plotted for all used solutions in Fig. 5.12(b). The enhancement is maximal around $h=3 \mathrm{~nm}$. Between 3 and $30 \mathrm{~nm}$, it decreases gradually with increasing film thickness. Below $3 \mathrm{~nm}$, the enhancement drops rapidly. The solution of $\mathrm{pH} 9.3 / 10$ $\mathrm{mM}$ gives the maximum enhancement, which is approximately a factor two. So, also in this case the calculated effect is of the same order of magnitude but slightly smaller than the measured increment. Qualitatively, the $c_{v e}$ coefficient differs from the measured $\gamma_{\text {int }} / \gamma_{\text {ref }}-1$. However, the length scale matches better, the maximal enhancement is observed close to $\kappa h<1$. On the other hand the concentration dependence for large distances is more off. Hence, both the electro-viscous and visco-electric effect only partially explain the observed dissipation behavior.

\subsection{Discussion}

Using the expressions presented in last section, we calculated for each considered fluid composition the dissipation $\gamma_{\mathrm{int}}(h)$. The input data for the calculations are the same as for the surface charge determination and have been listed in Table 5.1. The results have been plotted as $1 / \gamma_{\text {int }}$ versus $h$ in Fig. 5.9(b), which should be compared with the experimental results in Fig. 5.9(a). From both plots we observe that for large separations the dissi- 


\begin{tabular}{rl|rrrr}
{$[\mathrm{NaCl}]$} & {$[\mathrm{mM}]$} & $\delta_{\text {exp }}$ & $\delta_{\text {calc }}$ & $\kappa^{-1}$ & {$[\mathrm{~nm}]$} \\
\hline 0.2 & & 4.5 & 38 & 18 & \\
1 & 3 & 16 & 10 & \\
10 & $<1$ & 6 & 3 &
\end{tabular}

Table 5.2: The effective thickness $\delta$ of the enhanced-dissipation-layer in relation to the Debye length $\kappa^{-1}$ for the three salt concentrations used at $\mathrm{pH}=$ 9.3; for $\mathrm{pH} 5.7 / 1 \mathrm{mM}$ we obtain the same results as for $\mathrm{pH} 9.3 / 1 \mathrm{mM}$.

pation increases with decreasing concentration, while at close distances this trend vanishes. This can be explained from the thickness of the diffuse double layer, characterized by the Debye length, which increases with decreasing concentration (ionic strength). At large separations the double layers do not overlap and due to the higher friction in the double layer, the flow restricts itself to the middle region of the gap avoiding the double layer. This middle region is broader for high concentrations, leading to a lower flow resistance or dissipation. On the other hand at close distances, the double layers do overlap, so the flow is forced trough the double layer and the enhancement is not determined by the Debye length any longer. This effect has also been observed for flowing suspensions of colloidal particles which where coated with a dense polymer brush [53-55]. At low concentrations the flow avoids the brushes and the viscosity is determined by the volume fraction based on the dressed particle diameter including the polymer brush, while at high concentrations the flow is forced trough the brushes and the viscosity is determined by the bare particle volume fraction. By extrapolating the large distance behavior in Fig. 5.9 towards $1 / \gamma_{\text {int }}=0$, we obtain the effective thickness $\delta$ of the double layer both from our experiments and calculations (see also Appendix A). The results have been collected in Table 5.2. Indeed, both the experimental and calculated values for $\delta$ scale with the Debye length, but the calculations overestimate the thickness by approximately a factor 5 .

To get a better view on the dissipation enhancement at small tip-substrate distances, we plot $c_{\mathrm{ev}}=\gamma_{\mathrm{int}} / \gamma_{\mathrm{ref}}-1$ as a function of $h$ in Fig. 5.10(a) for the measured $c_{\mathrm{ev}-\mathrm{m}}$ and in Fig. 5.10(b) for the calculated $c_{\mathrm{ev}}$. From the calculations, we see that in this regime the enhancement in dissipation depends on both the surface charge and the Debye length. The enhancement is not monotonic with respect to the tip-substrate distance. It shows a maximum at a distance of approximately 1.5 times the Debye length, i.e. $\kappa h \approx 1.5$. These maxima in $c_{\mathrm{ev}}$ range from 0.6 for $10 \mathrm{mM}$ to 1.2 for $0.2 \mathrm{mM}$. For 
$\kappa h>2$, the enhancement decreases with a decay length approximately equal to $10 \kappa^{-1}$. At small distances for $\kappa h<1.5$, the enhancement drops rapidly. There are two reasons for this decrease. The "zero field" dissipation increases dramatically, $\gamma_{\text {int }} \sim h_{\text {local }}^{-3}$ while the excess ion density increases only with $\left(n^{+}-n^{-}\right) \sim h_{\text {local }}^{-1}$, where $h_{\text {local }}$ is the local height in the gap. Moreover, charge regulation dramatically decreases both the surface charge and the excess ion density in the gap, when $h \rightarrow 0$. This behavior is qualitatively in agreement with our experimental observations. However, quantitatively several deviations are observed. Although the order of magnitude is correct the experimentally observed maximal enhancement is larger than the calculated one. Moreover, the experimental maxima appear at smaller distances than the calculated ones, specially at the lowest concentration. Looking at the $c_{\mathrm{ev}}$ values for large $h$, experiment and calculation show the same trend: the $c_{\mathrm{ev}}$ for 10 and $1 \mathrm{mM}$ are approximately the same while the $0.2 \mathrm{mM}$ solution shows a clearly lower value. However, for $h<10 \mathrm{~nm}$ experiment and calculation show different trends: experimentally the $\mathrm{pH} 9.3 / 1 \mathrm{mM}$ solution shows the largest effect while the calculation shows the largest effect for the $\mathrm{pH}$ 9.3/0.2 $\mathrm{mM}$ solution.

\section{Earlier approaches}

The hydrodynamic drag force for two charged surfaces that approach each other in an electrolyte solution, has been investigated theoretically, [56-58] considering the ionic concentration dynamics in more detail. In these approaches the excess ions are not considered to be trapped, but the squeezingout flow of the ions in the electrolyte film is opposed by an inward electroosmotic flow to conserve the charge neutrality in the film. This osmotic flow is driven by a streaming potential, which is established by the initial outflow of ions.

Chun and Ladd give a detailed analysis [56], including the case of two parallel plates. In their analysis, the system is considered quasi-static and the narrow gap approximation is applied, using the same arguments as we have given above. They also obtained a numerical estimation for $c_{e v}$ for two plates in $\mathrm{NaCl}$ solutions, considering both constant charge and constant potential as boundary conditions for the PB equation. The different boundary conditions have only a minor effect on the calculated $c_{e v}$ values. Their results show that the electro-viscous coefficient increases gradually when the gap height decreases from $\kappa h=10$ to $\kappa h=3$ to $c_{e v}=0.4$. It decreases sharply when the separation is further reduced from $\kappa h=3$, and it approaches zero when $\kappa h \rightarrow 0$. The maximum enhancement $(<50 \%)$ is much smaller than our 
experimental observation. If we consider a parabolic tip on a flat surface, the electro-viscous coefficient $c_{e v}$ is expected to be smaller, as shown in Ref. [57] for the case of two spherical particles and the deviations between predictions and measurements become even larger. Hence our $c_{e v}$ calculations are in line with this more elaborated modelling, which is also not able to explain the enhanced dissipation quantitatively.

\section{Comparing a colloidal probe with a small flattened tip}

The force gradient measured with a small tip is also fitted with the DLVO theory, see Fig. 5.6 (a). The interaction area is approximated to be a round disk with a radius $R=40 \mathrm{~nm}$. The resulting surface charge has been plotted in Fig. 5.6 (c). The concentration and $\mathrm{pH}$ dependence of the surface charge are in agreement with the results obtained with the colloidal probe. The values of the surface charge are typically $50 \%$ lower than those measured with the colloidal probe. This may be caused by slight differences in the experimental procedures. For instance, the small tip is flattened in situ, which may result in different site density of surface groups from the tip side.

The damping measured with a small flattened tip is also compared with pure hydrodynamic dissipation, see Fig. 5.6 (b). In the calculations we consider the tip as a flat round disk or as spherical, both with a radius of $R=40$ nm. For a flat disk, instead of using Eq. 5.19, the damping coefficient is expressed as $\gamma_{\mathrm{int}}=1.5 \pi \eta_{b} R^{4} / h^{3}$.

The experimental data qualitatively show a distance dependence similar to that of a flat disk. The deviation may originate from the complex geometry of the real tip (see the inset in Fig. 5.6). More importantly, the enhancement in dissipation is reproduced. In line with the colloidal probe measurements, the dissipation at $5 \mathrm{~nm}$ is enhanced by approximately 100 to $200 \%$, see Fig. 5.6(d). Because the colloidal probe measurements already deviate a lot from the theoretical predictions, the dissipation enhancement measured with a small flattened tip is not compared with any theory.

\section{Ionic dissipation in the Stern layer}

Neither the electro-viscous nor the viscous-electric effect could explain the enhanced dissipation in overlapping EDLs qualitatively. What else could explain this enhancement?

In our analysis, we assume that the Stern layer is rigid, i.e. at the zero separation the Stern layers of the two surfaces are in contact with each other, and the ions in the Stern layer are considered as immobile. This assumption 
may not hold. In colloid science, surface conductance is often invoked to reconcile the measured surface potential or streaming potential with theoretical calculations $[14,29,59,60]$. It is plausible that the ions in the Stern layer are quite mobile. Hence, the Stern layer may yield under stress, and can be penetrated by the tip near zero separation. Given the fact that the Stern layer usually embodies much more ions than the diffuse layer, the ionic friction in the Stern layer may contribute considerably to the overall energy dissipation. In power generation measurements with pressure driven flow in nanochannels, the efficiency of the energy conversion is often much lower than expected. The excessive dissipation is then attributed to ionic friction in the Stern layer [59].

\section{Viscosity of interfacial water}

Another plausible explanation is that the water in the proximity of the charged surface is differently structured which can lead to a significant enhancement of its viscosity. Enhancement due to polarization effects was already considered in the visco-electric enhancement, but it is well-known that interfacial water forms also a layered structure in which the water molecules have a preferred orientation. This layering effect can increase the enhancement even more. Layering is consistently observed in SFA and AFM measurements $[1,10]$ and X-ray spectroscopy [33]. Moreover, the sum frequency generation (SFG) spectroscopy has revealed that the dipole moment of water molecules aligns with the electric field at the interface [61]. A stronger electric field leads to a more pronounced orientation [62], restricting the molecules in their rotation and therefore to a larger viscosity. Recently, Versch et al. [63] reported that the viscosity of water at a charged electrode could be magnified up to a few orders by increasing the surface charge. This is evidenced by the exceptionally slow Brownian diffusion of nano particles near the charged interface [63]. These interfacial water layers could be present in our system as well. It is possible to estimate the thickness of these layers by assuming that they are immobile, i.e. and show a negative slip length. For a $\mathrm{pH} \mathrm{9.3/0.2}$ $\mathrm{mM}$ solution, which shows the most pronounced enhancement in dissipation, the stagnant layer is approximately $2.5 \mathrm{~nm}$ thick at most on both the tip and the substrate. This value is in the same range as the reported thickness of 'superviscous' interfacial water [64]. 


\section{Uncertainty in 'zero' distance and finite $\gamma_{\text {int }}$ at the largest separa- tion}

The experimental uncertainty in the assumed 'zero' is approximately $0.5 \mathrm{~nm}$. For our reference case ( $\mathrm{pH} 5.7 / 100 \mathrm{mM})$, the bulk viscosity is nicely recovered without introducing an offset in the tip-substrate separation. It means that the 'zero' is determined in good accuracy. Moreover, we observe that the force gradient $\left(k_{\text {int }}\right)$ strongly decreases within $1 \mathrm{~nm}$ from the substrate, due to the van der Waals contribution, which dominates at these short distances. Note that the dissipation data measured with a colloidal probe $\left(\gamma_{\text {int }} \propto 1 / h\right)$ are less sensitive to the error in separation than those measured with a small flattened tip $\left(\gamma_{\text {int }} \propto 1 / h^{3}\right)$. Therefore, we give more credit to the data measured with a colloidal probe.

However, for a colloidal probe the dissipation enhancement is sensitive to a minor error in the calibration of $\gamma_{c}$ as discussed in section 5.3.2. It limits our discussion on the data measured close to the surface. This problem does not exist for a small flattened tip, because the dissipation decays faster with separation. As shown in Fig. 5.6(b), the linear relationship between $\log \gamma_{\text {int }}$ and $\log h$ holds even at the largest separations $(15 \mathrm{~nm})$ without correcting for a finite $\gamma_{\text {int }}$ value, as we did for the colloidal probe.

\subsection{Conclusion}

We have studied the hydrodynamic dissipation in overlapping electric double layers, using AFM amplitude modulation force spectroscopy. The AFM study has a unique advantage over conventional approaches. It enables independent and simultaneous determination of both the surface charge and the hydrodynamic dissipation as a function of the tip-substrate distance by analyzing the conservative and dissipative part of the measured force-distance curves. Our analysis shows that with AFM force spectroscopy one can measure the surface charge accurately.

From force measurements in various fluid compositions, we observe that the viscous dissipation is correlated with the surface charges on tip and substrate as well as the ionic strength (e.g. Debye length) in the electrolyte film. Using the measured surface charges, the enhancement in dissipation is numerically calculated from the friction due to the excess ions in the diffuse layer. The experimental data agree qualitatively with the calculations, the order of magnitudes are correctly reproduced, but the characteristic length scales do not match. Previous theoretical studies, taking into account the 
full dynamics of the ion distribution in the electrolyte film, corroborate our calculations and do not explain the deviations between experiment and calculation. To reconcile the disagreement, the visco-electric effect has been taken into consideration. Consequently, the characteristic length scales match quite well with the observations, but the visco-electric effect does not explain all observations quantitatively.

Our analysis shows that the description of an electric double layer using a mean field approach may be not sufficient when it comes to electro-hydrodynamic dissipation. We suggest that the interfacial water layer at a charged surface, a few nanometers thick, is superviscous and/or the conductance of the Stern layer may contribute substantially to viscous dissipation. However, the complex interplay between surface charge, structure of interfacial water layers and surface conductance is far from resolved, yet.

\section{Acknowledgement}

We thank Prof. Hans Lyklema for interesting discussions about this topic. This work has been supported by the Foundation for Fundamental Research of Matter (FOM), which is financially supported by the Netherlands Organization for Scientific Research (NWO).

\section{A Appendix}

\section{A: Description of the actual dissipation calculation}

To perform the actual calculation we make Eq 5.23 dimensionless:

$$
\partial_{\zeta}^{2} w_{r}=\frac{h^{2} \partial_{r} p}{\eta u_{0}}+|\sinh (\psi)| \frac{2 n_{\infty} k_{B} T h^{2}}{\eta D_{\text {ion }}} w_{r}
$$

where $\psi=e \phi / k_{B} T, w_{r}=u_{r} / u_{0}$ and $\zeta=z / h$. Defining:

$$
\frac{h^{2} \partial_{r} p}{\eta u_{0}}=-1
$$

and

$$
\frac{\eta D_{\text {ion }}}{2 n_{\infty} k_{B} T}=\delta^{2}
$$

we end up with:

$$
\partial_{\zeta}^{2} w_{r}=(h / \delta)^{2}|\sinh (\psi)| w_{r}-1
$$


We can write the solution of this equation formally as $w_{r}(\zeta)=f(\zeta, h)$, where $w_{r}(0)=w_{r}(1)=0$ meets the no slip boundary conditions. Appendix $\mathrm{C}$ describes how to do this. Then $\left\langle w_{r}\right\rangle=\int_{0}^{1} f(\zeta, h) d \zeta$ and

$$
\left\langle u_{r}\right\rangle=\frac{-h^{2} \partial_{r} p}{\eta} \int_{0}^{1} f(\zeta, h) d \zeta=\frac{-r U_{0}}{2 h}
$$

where $U_{0}$ is the speed of the probe. Hence we obtain:

$$
\partial_{r} p=\frac{\eta r U_{0}}{2 h^{3} \int_{0}^{1} f(\zeta, h) d \zeta}
$$

and with Eq. (5.24):

$$
\gamma_{\text {int }}=\frac{F_{\text {diss }}}{U_{0}}=\frac{1}{2} \pi \eta \int_{0}^{\infty} \frac{r^{3} d r}{h^{3} \int_{0}^{1} f(\zeta, h) d \zeta}
$$

We will analyze the dissipation assuming a spherical tip and a flat substrate. So Eq. (a2) can be written as:

$$
\gamma_{\text {int }}=\frac{F_{\text {diss }}}{U_{0}}=\pi \eta R_{\text {tip }}^{2} \int_{h_{0}}^{\infty} \frac{\left(h-h_{0}\right) d h}{h^{3} I(h)}
$$

where $I(h)=\int_{0}^{1} f(\zeta, h) d \zeta$. Last equation is identical with Eq. (5.25).

Note 1: Both $\delta$ and $\kappa$ depend on the ionic strength $n_{\infty}$, in such way that:

$$
\kappa \delta=\sqrt{\frac{e^{2} \eta D_{\text {ion }}}{\varepsilon \varepsilon_{0}\left(k_{B} T\right)^{2}}} \simeq 1.5
$$

Note 2: In case no surface charges are present, i.e. for $\psi(z)=0$, the flow profile is just parabolic: $\partial_{\zeta}^{2} w_{r}=-1$. Hence, $f(\zeta, h)=\frac{1}{2}\left(\zeta-\zeta^{2}\right)$ and we get the well known result:

$$
F_{\mathrm{diss}}=\pi \eta U_{0} \int_{0}^{\infty} \frac{r^{3} d r}{h^{3} \int_{0}^{1}\left(\zeta-\zeta^{2}\right) d \zeta}=6 \pi \eta U_{0} \int_{0}^{\infty} \frac{r^{3} d r}{h^{3}}
$$

For parallel disks this reduces to:

$$
F_{\text {diss }}=\frac{3 \pi \eta U_{0} R^{4}}{2 h^{3}}
$$

while for a sphere-plate geometry, characterized with $h=h_{0}+\frac{1}{2} r^{2} / R_{\mathrm{tip}}$, we obtain the Reynolds dissipation force:

$$
F_{\text {diss }}=6 \pi \eta U_{0} \frac{R_{\text {tip }}^{2}}{h_{0}}
$$




\section{B: Calculation of the $c_{v e}$ coefficient}

According to Lyklema [34] the viscosity of water depends on the electric field strength according:

$$
\eta(E)=\eta_{0}\left(1+f_{v e} E^{2}\right)
$$

where $f_{v e} \approx 10^{-15} \mathrm{~m}^{2} / \mathrm{V}^{2}$ is the visco-electric coefficient. As the field strength varies with position we must consider the position dependence of the viscosity when solving the continuity and Navier-Stokes equations:

$$
\nabla \cdot \underline{u}=0
$$

and:

$$
\rho\left(\frac{\partial \underline{u}}{\partial t}+\underline{u} \cdot \nabla \underline{u}\right)=-\nabla p+\eta \nabla^{2} \underline{u}+2(\nabla \eta) \cdot \underline{\underline{D}}-e\left(n^{+}-n^{-}\right) \nabla \phi
$$

while the stress tensor itself is given by:

$$
\underline{\underline{\tau}}=-p \underline{\underline{\delta}}+2 \eta \underline{\underline{D}}+\varepsilon \varepsilon_{0}\left(\nabla \phi \nabla \phi-\frac{1}{2}(\nabla \phi)^{2} \underline{\underline{\delta}}\right)
$$

where $\underline{\underline{D}}=\frac{1}{2}\left(\nabla \underline{u}+(\nabla \underline{u})^{T}\right)$ is the rate of strain tensor. We investigate these relations in a parallel plate geometry in the limit $h / R \ll 1$. Here $h(r)$ is the gap height and $R$ the characteristic radial distance. Neglecting inertia and the $\partial_{r} \phi$ term, Eq. (a7) reduces to:

$$
\partial_{r} p=\partial_{z}\left(\eta \partial_{z} u_{r}\right)
$$

while the continuity relation reads:

$$
\left\langle u_{r}\right\rangle(r)=\frac{1}{h} \int_{0}^{h} u_{r}(r, z) d z=\frac{-r U_{0}}{2 h(r)}
$$

where $U_{0}$ is the velocity of the upper surface (i.e. the tip). To continue, we rewrite Eq. (a9) in dimensionless form as:

$$
\partial_{\zeta}\left(\eta_{r} \partial_{\zeta} w_{r}\right)=\frac{h^{2} \partial_{r} p}{\eta_{0} u_{0}}
$$

with $\zeta=z / h, \eta_{r}=\eta / \eta_{0}$ and $w_{r}=u_{r} / u_{0}$. Because $\partial_{z} \partial_{r} p=0$ we can choose $u_{0}=-h^{2} \partial_{r} p / \eta_{0}$ and write last equation as:

$$
\partial_{\zeta}\left(\eta_{r} \partial_{\zeta} w_{r}\right)=-1
$$


The solution is again formally given by $w_{r}=g(\zeta, h)$ and $\left\langle w_{r}\right\rangle=\int_{0}^{1} g(\zeta, h) d \zeta$. Because $\left\langle u_{r}\right\rangle=-h^{2} \partial_{r} p / \eta_{0}\left\langle w_{r}\right\rangle$, we obtain for the pressure gradient:

$$
\partial_{r} p=\frac{\eta_{0} r U_{0}}{2 h^{3} \int_{0}^{1} g(\zeta, h) d \zeta}
$$

which is, when one replaces $g(\zeta, h)$ with $f(\zeta, h)$, identical with Eq. (a3). Hence, also the expression for $\gamma_{\text {int }}$ is given again by :

$$
\gamma_{\text {int }}=\frac{F_{\text {diss }}}{U_{0}}=\pi \eta R_{\text {tip }}^{2} \int_{h_{0}}^{\infty} \frac{\left(h-h_{0}\right) d h}{h^{3} I(h)}
$$

where in this case $I(h)=\int_{0}^{1} g(\zeta, h) d \zeta$.

To take into account both the electro-viscous and the visco-electric effect we can use the same approach and generalize our result by considering $g(\zeta, h)$ as the solution of:

$$
\partial_{\zeta}\left(\eta_{r} \partial_{\zeta} w_{r}\right)=(h / \delta)^{2}|\sinh (\psi)| w_{r}-1
$$

where in this case $\delta^{2}=\eta_{0} D_{\text {ion }} /\left(2 n_{\infty} k_{B} T\right)$. Again, $\gamma_{\text {int }}$ can be calculated with Eq. (a14).

\section{C: Solution of the differential equations for $\mathrm{w}_{\mathbf{r}}(\zeta)$}

Because Eqs. (a1) and (a12) are equal to Eq. (a15), for $\eta_{r}=1$ and $\delta^{-1}=0$, respectively, we only need to describe how to solve last equation. To do so, we define $q(\zeta)=\eta_{r} \partial_{\zeta} w_{r}$ and rewrite the second order differential equation as two first order equations:

$$
\begin{gathered}
\partial_{\zeta} w_{r}=q / \eta_{r} \\
\partial_{\zeta} q=(h / \delta)^{2}|\sinh (\psi)| w_{r}-1
\end{gathered}
$$

This set is solved using an iterative Runge Kutta integration scheme, with the boundary conditions $w_{r}(0)=0$ and $q(0)=q_{\text {guess }}$. In the iteration process we optimize $q_{\text {guess }}$ in such a way that eventually the second boundary condition for $w_{r}$, i.e. $w_{r}(1)=0$, is fulfilled, too. This is achieved by taking an upper and lower limit for $q_{\text {guess }}$, take $q_{\text {guess }}$ as the average of these two limits and calculate $w_{r}(1)$ by applying the integration routine. If $w_{r}(1)>0$ we replace the upper limit by $q_{\text {guess }}$, otherwise the lower limit by $q_{\text {guess }}$ and repeat the process. In this way we restrict the possible values for $q_{\text {guess }}$ to an interval that decreases every iteration by a factor two, until the difference between upper and lower limit is small enough. 


\section{Bibliography}

[1] Israelachvili J 1991 Intermolecular and Surface Forces 2nd ed (Academic press, London)

[2] Park N, Kwon B, Kim I S, and Cho J W 2005 J. Membrane Sci. 258 43

[3] Kumar N, Wang L, Siretanu I, Duits M, and Mugele F 2013 Langmuir 295150

[4] Mugele F, Bera B, Cavalli A, Siretanu I, Maestro A, Duits MHG, CohenStuart M, van den Ende D, Stocker I, and Collins I 2015 Sci. Rep. 5 10519

[5] Lyklema J 1994 Colloids Surfaces A: Physicochem. Eng. Aspects 9241

[6] Hillier A C, Kim S, and Bard A J, 1996 J. Phys. Chem. 10018808

[7] Sparreboom W, van den Berg A, and Eijkel J C T 2009 Nature Nanotechnology 4713

[8] Zhmud B V, Meurk A, and Bergstrom L 2000 Colloids and Surfaces A: Physicochem. Eng. Aspects $\mathbf{1 6 4} 3$

[9] Zhao C, Ebeling D, Siretanu I, van den Ende D, and Mugele F 2015 Nanoscale Doi: 10.1039/C5NR05261K

[10] Liu F, Zhao C, Mugele F, and van den Ende D 2015 Nanotechnology 26 385703

[11] Bonthuis D J and Netz R R 2013 J. Phys. Chem B. 11711397

[12] Siretanu I, Ebeling D, Andersson M P, Stipp S L S, Philipse A, Cohen Stuart M, van den Ende D, and Mugele F 2014 Sci. Rep. 44956

[13] Huang D M,Cottin-Bizonne C,Ybert C, and Bocquet L2007 Phys. Rev. Lett. 98177801

[14] Bocquet L and Charlaix E 2010 Chem. Soc. Rev. 391073

[15] Rubio-Hernándeza F J, Carriqueb F, and Ruiz-Reinaa E 2004 Adv. Colloid Interface Sci. 51107 
[16] van der Heyden F H J, Bonthuis D J, Stein D, and Meyer C, Dekker C 2006 Nano Lett. 62232

[17] Yang J, Lu F, Kostiuk L W, and Kwok D Y 2003 J. Micromech. Microeng. 13963

[18] Daiguji H, Yang P D, Szeri A J, and Majumdar A 2004 Nano Lett 4 2315

[19] Sparreboom W, van den Berg A, and Eijkel J C T 2009 Nature Nanotechnology 4713

[20] Wang M R and Kang Q J 2010 Microfluid. Nanofluid. 9181

[21] Xie Y B, Sherwood J D, Shui L L, van den Berg A, and Eijkel J C T 2011 Lab Chip 114006

[22] Levine S, Marriott J R, and Robinson K 1975 J. Chem. Soc., Faraday Trans. 2 711

[23] Booth F 1950 Proc. Roy. Soc. A 203533

[24] Tas N R, Haneveld J, Jansen H V, Elwenspoek M, and van den Berg A 2004 Appl. Phys. Lett. 853274

[25] Haneveld J, Tas N R, Brunets N, Jansen H V, and Elwenspoek M 2008 Appl. Phys. Lett. 104014309

[26] Mortensen N A and Kristensen A 2008 Appl. Phys. Lett. 92063110

[27] Hunter R J, Ottewill R H, and Rowell R L 1981 Zeta Potential in Colloid Science (Academic press, London)

[28] Russel W B, Saville D A, and Schowalter W R Colloidal Dispersions 1989 Cambridge University Press, Cambridge

[29] Delgado V A, González-Caballero F, Hunter R J, Koopal L K, and Lyklema J 2005 Pure Appl. Chem. 771753

[30] Chang C C, Yang R J, Wang M, Miau J J, and Lebiga V 2012 Phys. Fluids 24072001

[31] Huang D, Cottin-Bizonne C, Ybert C , and Bocquet L 2008 Phys. Rev. Lett. 101064503

[32] Bazant M Z, Kilic M S, Storey B D, and Ajdari A 2009 Adv. Colloid Interface Sci. 15248

[33] Cheng L, Fenter P, Nagy K L, Schlegel M L, and Sturchio N C 2001 Phys. Rev. Lett.

[34] Lyklema J and Overbeek J Th G 1961 J. Colloid Interface Sci. 16501

[35] Andrade E N, Da C, and Dodd C 1946 Proc. Roy. Soc. (London) A 187 296

[36] Hunter R and Leyendekkers J 1978 J. Chem. Soc., Faraday Trans. 174 450 
[37] Li D Q 2001 Colloid Surf A 19135

[38] Washburn E W 1921 Phys. Rev. 17273

[39] Oh J M, Taez T, de Beer S, and Mugele F 2010 Microfluid Nanofluid 9 123

[40] Siria A, Poncharal P, Biance A L, Fulcrand R, Blase X, Purcell S T, and Bocquet L 2013 Nature 455494

[41] Cottin-Bizonne C, Cross B, Steinberger A, and Charlaix E 2005 Phys. Rev. Lett. 94056102

[42] Labuda A, Cleveland J, Geisse N A,Kocun M, Ohler B, Proksch R, Viani M B, and Walters D 2014 Microscopy and Analysis 2821

[43] Cleveland J P, Anczykowski B, Schmid A E, and Elings V B 1998 Appl. Phys. Lett. 722613

[44] Garcia R and Perez R 2002 Surf. Sci. Rep. 47197

[45] de Beer S, van den Ende D, and Mugele F 2011 J. Phys.: Condens. Matter 2311206

[46] de Beer S, van den Ende D, and Mugele F 2010 Nanotechnology 21 325703

[47] de Beer S, van den Ende D, and Mugele F 2008 Appl. Phys. Lett. 93 253106

[48] Butt H J and Jaschke M 1995 Nanotechnology 61

[49] Sader J E, Uchihashi T, Higgins M J, Farrell A, Nakayama Y, and Jarvis S P 2005 Nanotechnology 16 S94

[50] Hiemstra T, De Wit J C M, and Van Riemsdijk W H 1989 J. Colloid Interface Sci. 133105

[51] Behrens S H and Grier D G 2001 J. Chem. Phys. 1156716

[52] Cottin-Bizonne C, Cross B, Steinberger A, and Charlaix E 2005 Phys. Rev. Lett. 94056102

[53] Nommensen P A, Duits M H G, van den Ende D, and Mellema J 1999 Phys. Rev. E 593147

[54] D'Haene P 1992 Ph.D. Thesis, Katholieke Unversiteit Leuven, Belgium

[55] Neuhausler S and Richtering W 1995 Colloids Surf., A 9739

[56] Chun B and Ladd A C 2004 J Colloid. Interf. Sci. 274687

[57] Muller V M 1990 J. Colloid Interface Sci. 13661

[58] Warszynski P and Vandeven T G M 1990 Faraday Discuss. 90313

[59] van der Heyden F H J, Bonthuis D J, Stein D, Meyer C, and Dekker C 2007 Nano Lett. 71022

[60] Lyklema J 2001 J. Phys.: Condens. Matter 135027 
[61] Nihonyanagi S, Yamaguchi S, and Tahara T 2009 J. Chem. Phys. 130 204704

[62] Lis D, Backus E H G, Hunger J, Parekh S H, and Bonn M 2014 Science 3441138

[63] Verch A, Pfaff M, and de Jonge N 2015 Langmuir 316956

[64] Guriyanova S, Mairanovsky V G, and Bonaccurso E $2011 \mathrm{~J}$. Colloid Interface Sci. $\mathbf{8 0 0} 360$ 


\section{Summary}

Solid-liquid interfaces are not only omnipresent in our daily lives, but also in many applications in industry and technology. However, our fundamental understanding of the physical properties of these interfaces is far from complete. In this thesis, we study properties, such as solvation forces, DLVO forces and electro-hydrodynamic dissipation of solid-liquid interfaces by the use of AFM. Thereby, we study various methods of AFM force spectroscopy, to find reliable and efficient measurements under liquid. Techniques explored include thermal noise spectroscopy (TNS), amplitude modulation (AM) with piezo excitation and photothermal excitation. With these techniques, we investigate solvation forces in non-polar liquids and water, DLVO forces, and electro-hydrodynamic dissipation in the electric double layer (EDL).

A liquid confined between two solid surfaces is a topic of intense research in geoscience, tribology, and biophysics. The AFM, as an important tool for nanoscopic force measurements, has been used to probe the viscoelastic dynamics of nano confined liquids. The discrepancies between results obtained with different AFM methods are often attributed to various technical reasons, such as incomplete understanding of the dynamics of a cantilever under liquid and differences in tip geometry. In Chapter 3, we explore TNS in the context of nano confined liquids. By measuring the thermal fluctuations of a cantilever, we measure solvation forces and hydrodynamic dissipation in confined liquids. In TNS, the cantilever dynamics under liquid are approximated as a simple harmonic oscillator (SHO). We show, theoretically and experimentally, that the approximation is sufficiently accurate within the experimental error in practical cases, for instance the viscosity of a non-polar liquid is measured with an accuracy of approximately $20 \%$. With a nanoscopic tip, we observe oscillatory solvation forces and non-monotonic dissipation, which can be correlated to a layered structure of confined liquids. Our results are in line with the idea that the continuum theory breaks down when it comes to confined liquid films of molecular thickness. Despite all of its strength, TNS 
is slow and its accuracy goes down dramatically in an overdamped system.

The AM force spectroscopy with piezo excitation, faster than TNS, is one of the most widely used methods. Its reliability under liquid is open to discussion, in particular, when it comes to quantitative measurements. In Chapter 4, the reliability of piezo-excited AM in comparison with TNS is investigated. In the data analysis of piezo-excited AM, both the conventional point-mass model and the recently proposed continuum beam approach are examined through the measurements of DLVO forces, solvation forces, and the hydrodynamic dissipation in aqueous electrolyte solutions. Our results show that piezo-excited AM (the continuum beam approach) is reliable if the fluid excitation is taken into account. The point-mass model fails qualitatively for determining the dissipative part of the interactions. In this study, we also observe that the dissipation in the hydration layers is non-monotonic with respect to thickness, which reflects the discrete nature of the hydration films. Moreover, we observe that the hydrodynamic dissipation in the EDL is enhanced substantially, compared to the bulk.

Understanding the coupling between electrostatics and hydrodynamics is important for nanofluidics and colloid science. Conventional techniques often only allow us to measure either the electrostatic force or the dissipation. To overcome this problem, we propose to investigate electro-hydrodynamics with AFM in Chapter 5. The measurements are performed with aqueous electrolytes at different $\mathrm{pH}$ levels and concentrations, such that the surface charge and screening strength are adjusted. From the data obtained in the case of strong screening, the viscosity of the bulk liquid is accurately derived, which shows the validity of our approach. From electrostatic forces, we obtain the charge distribution in the gap between tip and substrate by solving the Poisson-Boltzmann equation with charge regulation boundary conditions. We observe that the viscous dissipation is correlated with the charge density in the diffuse layer. The observed enhancement cannot be successfully rationalized according to the theories on the electro-viscous effect or the visco-electric effect, but can only be qualitatively explained. It may be due to the poorly understood ionic transport near a charged surface or the increased viscosity of interfacial water. 


\section{Samenvatting}

Grensvlakken tussen vaste stoffen en vloeistoffen komen we niet alleen veel tegen in ons dagelijks leven, maar ook in toepassingen van de industrie en wetenschap. Echter, ons fundamenteel begrip van de fysische eigenschappen van deze grensvlakken is ver van compleet. In dit werk bestuderen we eigenschappen zoals solvatatie krachten, elektrostatische krachten en elektrohydrodynamische dissipatie aan grensvlakken met een atomic force microscoop (AFM). We bestuderen ook verschillende technieken van AFM krachtenspectroscopie, om een betrouwbare en efficiënte manier te vinden van metingen doen in een vloeistof. De technieken die bestudeerd zijn, zijn thermische ruis spectroscopie (TNS), amplitude modulatie (AM) met piezo excitatie en fotothermische excitatie. Met deze technieken onderzoeken we solvatatie krachten in non-polaire vloeistoffen en water, DLVO krachten en elektrohydrodynamische dissipatie in de elektrische dubbellaag.

Er wordt in veel verschillende disciplines onderzoek gedaan naar het gedrag van vloeistof wat zich tussen twee oppervlakken bevindt. Voorbeelden zijn aardwetenschappen, wrijvingsleer, en biowetenschappen. De AFM, een belangrijke apparaat voor het doen van nanoscopische krachtmetingen, is gebruikt om elektro viskeuze eigenschappen van begrensde vloeistoffen te meten. De discrepantie die gevonden wordt tussen resultaten die verkregen zijn met verschillende AFM technieken, wordt vaak toegeschreven aan verscheidene technische redenen, zoals een incompleet begrip van cantilever dynamica in vloeistof of verschil in de tip geometrie. In hoofdstuk 3 verkennen we de TNS techniek in de context van nano begrensde vloeistoffen. Met de thermische fluctuaties van de cantilever, meten we de solvatatie krachten en de hydrodynamische dissipatie van begrensde vloeistoffen. In TNS wordt de beweging van de cantilever benaderd als een simpele harmonische oscillator (SHO). We laten theoretisch en experimenteel zien dat deze benadering voldoende nauwkeurig is binnen de experimentele fout in normale gevallen; de viscositeit van een non-polaire vloeistof kan binnen een $20 \%$ nauwkeu- 
righeid benaderd worden. Met een nanoscopische tip zien we oscillerende solvatatiekrachten en non-monotonische dissipatie, die gerelateerd zijn aan een georganiseerde structuur van de begrensde vloeistof. Onze resultaten zijn consistent met het idee dat de continuüm theorie niet meer geldt, omdat de begrensde vloeistof in de ordegrote is van een molecuul. Ondanks de duidelijke voordelen, is TNS erg langzaam en gaat de nauwkeurigheid omlaag in een over-gedempt systeem.

Amplitude modulatie met piezo excitatie is de meeste gebruikte techniek en is sneller dan TNS. De betrouwbaarheid in vloeistoffen is echter nog twijfelachtig, met name in het geval van kwantitatieve metingen. In hoofdstuk 4 vergelijken we de betrouwbaarheid van piezo geëxciteerde amplitude modulatie met de thermische ruis spectroscopie. In de data analyse van de piezo geëxciteerde AM worden beide het conventionele puntmassa model en het recent ontwikkelde continuüm model vergeleken met behulp van DLVO krachten, solvatatie krachten en hydrodynamische dissipatie in elektrolytische oplossingen. Onze resultaten laten zien dat piezo geëxciteerde AM (gebruik makende van het continuüm model) betrouwbaar is als er ook rekening wordt gehouden met de excitatie van de omringende vloeistof. Het puntmassa model kan niet gebruikt worden om het dissipatieve gedeelte van de interactie te beschrijven omdat deze kwalitatief niet klopt. In deze studie wordt ook aangetoond dat de dissipatie in hydratatielagen niet monotoon van karakter is in vergelijking met de dikte, wat de discrete natuur van de hydratatielagen toont. Bovendien, zien we dat de hydrodynamische dissipatie versterkt is in de elektrische dubbellaag vergeleken met de bulk.

Het begrijpen van de koppeling tussen elektrostatische krachten en hydrodynamica is belangrijk voor nanofluidics en colloidwetenschap. Conventionele technieken zijn vaak alleen in staat om de elektrostatische kracht of de dissipatie te meten. Om dit probleem op te lossen, gebruiken we AFM om elektrohydrodynamica te bestuderen, wat beschreven wordt in hoofdstuk 5. De metingen zijn gedaan in elektrolytische oplossingen met verschillende $\mathrm{pH}$ en zout concentraties. Uit de data waarbij de oppervlakte lading grotendeels geneutraliseerd is kan de viscositeit van de bulk vloeistof nauwkeurig bepaald worden, wat onze aanpak valideert. Van de elektrostatische krachten krijgen we de ladingsverdeling tussen de cantilever en substraat door de Poisson-Boltzmann vergelijking op te lossen met ladingsnormerende grensvoorwaarden. We zien dat de viskeuze dissipatie is gecorreleerd met de ladingsdichtheid in de diffusieve laag. De geobserveerde verhoogde elektroviskeuze dissipatie kan niet succesvol gerationaliseerd worden volgens de bestaande theorieën over elektro viskeuze effecten, maar kan alleen kwalitatief verklaard worden. Dit komt waarschijnlijk doordat het ionentransport langs 
een geladen oppervlak slecht voorspeld kan worden of doordat de viscositeit van het water aan het grensoppervlak verhoogd is. 



\section{Publications}

\section{Publications from this thesis}

1. Atomic force microscopy of confined liquids using the thermal bending fluctuations of the cantilever

Fei Liu, Sissi de Beer, Dirk van den Ende, and Frieder Mugele Phys. Rev. E 87, 062406, 2013 (link to pdf)

2. Amplitude modulation atomic force microscopy, is acoustic driving in liquid quantitatively reliable?

Fei Liu, Cunlu Zhao, Frieder Mugele, and Dirk van den Ende Nanotechnology, 26, 385703, 2015 (link to pdf)

3. Viscous dissipation in overlapping electric double layers

Fei Liu, Cunlu Zhao, Frieder Mugele, and Dirk van den Ende (under preparation)

\section{Other publications}

1. Stretching of Collapsed Polymers Causes an Enhanced Dissipative Response of PNIPAM Brushes near their LCST

Yunlong Yu, Bernard D. Kieviet, Fei Liu, Igor Siretanu, Edit Kutnyanszky, G. Julius Vancso, and Sissi de Beer

Soft Matter, 11, 8508, 2015 (link to pdf)

2. Surface charge heterogeneity of gibbsite nano-particles probed by atomic force microscopy

Aram Klaassen, Fei Liu, Igor Siretanu, Dirk van den Ende, and Frieder Mugele

(under preparation) 



\section{About the Author}

Fei Liu was born on April, $10^{\text {th }}, 1986$, in Jiangsu, China. Between 2005 and 2009, he studied for a B.Sc. degree in Electrical Engineering at Northwestern Polytechnical University in Xi'an, Shaanxi, China. He continued his study at University of Southern Denmark, Sonderborg. In 2011, he obtained his M.Sc. degree in Mechatronics. In August 2011, he became a PhD student in the group of Physics of Complex Fluids at the University of Twente, the Netherlands. His PhD project is the study of solid-liquid interfaces using Atomic Force Microscopy. 



\section{Acknowledgments}

A friend of mine told me that in this part the author is expected to describe how life was in the past $4+$ years before acknowledging people. I do not really know the proper words. It is pretty much like this: I walked through a forest to look for a lake with an out-of-date map in my hand. The tall and thick trees blocked my vision on most days. I was encouraged by fairies and trapped by monsters on the way. Occasionally, I questioned myself, 'What is the point in finding the lake? What if I had not start the walk? What if... '. Now I stand in front of a lake. The lake has nothing in common with what I imagined, but that does not matter any more. I had great memorable moments through the walk, and got help from a lot of people.

First of all, I would like to express my gratitude to my supervisor, Prof. Frieder Mugele. I appreciate his time, ideas and encouragement. It was always inspiring to discuss with him. He always had lots of ideas. After the discontinuation of the project on the water-graphene interface, I was guided to explore other subjects.

I am deeply indebted to my daily supervisor, Dr. Dirk van den Ende. Dirk is an amazing and sympathetic physicist. Without his guidance and unrelenting support, it would be impossible to achieve this $\mathrm{PhD}$. The theoretical part presented in this thesis would not have seen the day of light without him. He helped correcting manuscripts, over and over again. Conversations with him are not only about research but also about life. They really help alleviate my frustration and anxiety especially in the final stage of the $\mathrm{PhD}$, when I was confronted by overwhelming challenges in several occasions.

It is a great pleasure to work with Sissi de Beer. The passion she has for research is contagious. She taught me how to operate AFM in the first few months of the project. She proposed to measure the relaxation time of confined liquids as the first research question when I started the $\mathrm{PhD}$. Although the idea failed, because the time scale is beyond the resolution of AFM, I got to learn the topic of confined liquids in depth from her. Her PhD 
thesis is a guide book for me. In her thesis, she proposed the thermal noise approach. Her contributions tremendously improved the work presented in Chapter 3.

My thanks go to Igor for sharing with me his knowledge of surface chemistry and his wonderful $\mathrm{PhD}$ stories many times. I really like our trips together to the noncontact-AFM conferences in Maryland and Tsukuba. More importantly, you set a good example to me on how to plan an experiment and how to attract others to team up.

I thank my paranymphs, Aram and Simone, for their generous help in preparing the defense. They also helped improving the draft of this thesis and writing the Dutch samenvatting. Aram also designed the cover of this thesis. It was fun to do overnight AFM experiments with Aram before Christmas eve. Naveen, my lab manager, is always happy and easy-going. In the beginning of our $\mathrm{PhD}$, we tried many things together. Most of them failed, but we still learned a lot. Thanks for your introductory course to Bollywood music in the AFM lab. Together with Dirk, Cunlu came up with a theory for dissipation in the electric double layer. I also benefit from Cunlu's theoretical knowledge in extracting surface charge from AFM force-distance curves. It is very helpful to discuss with Lei some chemistry questions. The chat with Chinese friends during lunch is a lot of fun. The jokes told by Arjen in coffee breaks are hilarious although a few of them are offensive in a racist mind. They definitely raise my tolerance to racism. Thanks go to Arjen and Aram for reminding me of my Chinese accent. It is interesting to discuss non-academic topics with our PhD and post-Doc fellows (Martin, Bijoy, Kartikeya, Aditya, Stelian, Somnath, and Zhantao). Thank my officemates (Davood, Kartikeya, Stelian, Jolet, and Arun). Our office is often quiet, which suits me best. Our (former) secretaries (Isabel, Annelies, Edith, and Lisette) and lab engineers (Mariska, Carla, and Daniel) are super efficient in dealing with documents and requests. Daniel Hagedoorn and Daniel Wijnperle are awesome and very professional. Carla and Daniel(s) always set a very high standard in sorting out things and cleanliness in the lab.

I would like to thank Lantian Chang\&Ying Du for being a great host and hostess. Yanting and I felt really relaxed and comfortable at your place. Thank Zhonghua Chen\&Jingwei Zhang for being hospital, especially for offering accommodation and food when Yanting and I were locked out of our apartment. It is nice to meet my NWPU alumni-Yali Zhang\&Jie Zhao, Xian Qiu\&Yuan Feng, and Yanbo Xie in Twente. It is fun to play badminton with Dongfang Liu, Xuelong Chen, Xiaokui Zhang, Ding Zheng, Yun Lin, Yunlong Yu and Xiaohua Li. There is nothing better than smashes to release the stress from work. 
I am truly blessed to meet and marry Yanting in UT. Her love makes my world more lovely and relaxing. I am thankful to my parents and my parentsin-law for their support. I am grateful to my brother, my sister-in-law, and my nephew, especially for taking good care of my parents. 感谢我的妻子陈 燕婷。认识她让我对觉得这个世界更可爱, 更诙谐, 更轻松。感谢我的岳父 岳母的支持。感激我父母的养育之恩。感谢我的哥哥, 嫂子，和侄子可乐。 感谢他们对父母无微不至的照顾。

Fei Liu (刘飞), Enschede, May, 2016 



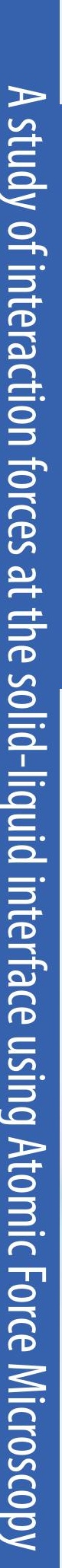

ISBN: 978-90-365-4131-2

$\frac{\text { D. }}{\text { 至. }}$ 